\title{
Acknowledgment to Reviewers of Molecules in 2020
}

\author{
Molecules Editorial Office
}

MDPI AG, St. Alban-Anlage 66, 4052 Basel, Switzerland

Peer review is the driving force of journal development, and reviewers are gatekeepers who ensure that Molecules maintains its standards for the high quality of its published papers. Thanks to the cooperation of our reviewers, in 2020, the median time to first decision was 13 days and the median time to publication was 32 days. The editors would like to express their sincere gratitude to the following reviewers for their precious time and dedication, regardless of whether the papers were finally published:

A. Bekhit, Alaa El Din

Aandres-Mach, Marta

Aav, Riina

Abarbri, Mohamed

Abashev, George G.

Abate, Lorenzo

Abba, Mohammed L.

Abballe, Annalisa

Abbate, Sergio

Abbehausen, Camilla

Abbrent, Sabina

Abbruzzetti, Stefania

Abd El Hakim, Yasmina

Abd El-Hack, Mohamed E.

Abdala, Ahmed

Abdallah, Shaaban

Abdel-Rahman, Laila H.

Abdel-Razek, Ahmed

Abdelwahed, Sameh

Abdullah, Oday Ibraheem

Abe, Naohito

Abebe, Worku

Åberg, Daniel N.

Abollino, Ornella

Abramova, Tatyana V.

Abramovich, Haim

Ábrányi-Balogh, Péter

Abrunhosa, Luís

Abugri, Daniel A.

Abuin, Graciela

Abu-Reidah, Ibrahim M.

Abushouk, Abdelrahman

Acero, Nuria

Aceto, Maurizio

Achelle, Sylvain

Achinivu, Ezinne
Achour, Brahim

Aci-Sèche, Samia

Ačkar, Đurđica

Acker, Jörg

Acquah, Caleb

Acquaviva, Rosaria

Ádám, Tölgyesi

Adam, Tomáš

Adamakis, Ioannis-Dimosthenis

Adamcová, Dana

Adamczyk, Greta

Adamczyk-Woźniak, Agnieszka

Adamkova, Anna

Adamo, Carlo

Adamopoulos, Stergios

Addepalli, Balasubrahmanyam

Adebo, Oluwafemi

Adelberg, Jeffery

Adewole, Deborah

Adi, Santoso Cornelia Melinda

Adil, Mohd

Adil, Syed Farooq

Adjé, Félix

Adly, Frady G.

Adt, Isabelle

Afantitis, Antreas

Afarinkia, Kamyar

Afify, Ahmed Sabry

Afonin, Kirill

Afonso, Carlos M.

Afseth, Nils Kristian

Agamennone, Mariangela

Aganovic, Kemal

Agartan, Lutfi

Agbossou-Niedercorn, Francine

Ageitos, Jose Manuel 
Agerbirk, Niels

Aggarwal, Bharat B.

Aggeli, Ioanna-Katerina

Agha, Ramsy

Aghazada, Sadig

Agić, Dejan

Agnieszka, Szopa

Agòcs, Attila

Agosta, Lorenzo

Agostino, Mark

Agrawal, Alok

Agrawal, Dinesh

Aguayo, Maria Graciela

Aguiar, André

Aguilar, Enrique

Aguilar-Bolados, Hector

Aguilar-Caballos, María Paz

Aguiló-Aguayo, Noemí

Aguirre, Nestor F.

Agusa, Tetsuro

Agustin, Dominique

Agustin, Melissa B.

Ahamed, Muneer

Ahlenstiel, Chantelle

Ahluwalia, Amrita

Ahmad, Javed

Ahmad, Khurshid

Ahmad, Zulfiqar

Ahmadi, Mojtaba

Ahmadivand, Arash

Ahmed, Alauddin

Ahmed, Hafiz

Ahmed, Khalil

Ahmed, Saveer

Ahmed, Sheikh Ali

Ahmedova, Anife

Ahn, Hee-Chul

Ahn, Ji-Young

Aiba, Yuichiro

Aiello, Francesca

Aiello, Gilda

Aimanianda, Vishu Kumar

Airaksinen, Anu

Aires, Alfredo

Aitken, R. Alan

Ajay, Amrendra K.

Ajay, Ashok

Akabayov, Barak

Akad, Fouad

Akbar, Mohammed

Åkerström, Bo
Akhgari, Amir

Akhrem, Irena

Akimoto, Seiji

Akita, Sadanori

Akitsu, Takashiro

Akiyama, Hisashi

Akopova, Tatiana

Akurathi, Gopalakrishna

Alaimo, Alessandro

Alam, Arif U.

Alam, Mohammad Abrar

Alam, Todd

Alander, Jarmo

Alarcon, Julio

Alarif, Walied Mohamed

Albalat, Amaya

Albano, Gianluigi

Albarracín, Sonia L.

Albeck, Amnon

Albericio, Fernando

Albero, Josep

Albertolle, Matthew E.

Albillos, Almudena

Albiniak, Philip

Albuquerque, Hélio

Alcantara, Andres

Alcaraz, Miguel

Alcarde, Andre Ricardo

Alcaro, Stefano

Alcolea Palafox, Mauricio

Alcolea, Veronica

Alcoutlabi, Mataz

Aldred, Katie

Aldrich-Wright, Janice

Alduina, Rosa

Al-Dujaili, Emad

Alegria, Elisabete C.B.A.

Aleixandre, Manuel

Alejandro-Martín, Serguei

Aleksandrov, Alexey

Alekseeva, Anna

Alen, Francisco

Aleshin, Andrey

Alessandro, Cecconello

Alessio, Nicola

Alexa-Stratulat, Teodora

Alexis, Frank

Al-Fatimi, Mohamed

Alfei, Silvana

Algieri, Vincenzo

Alguacil, Francisco 
Al-Horani, Rami A.

Ali, Hayssam M.

Ali, Naser

Ali, Rameez

Ali, Shafaqat

Aliabadian, Ehsan

Aliaño González, María José

Alikhani, Esmail

Alina, Brzeczek-Szafran

Alipieva, Kalina

Al-Khrasani, Mahmoud

Alkorta, Ibon

Allais, Florent

Allan, Sandra

Allegra, Mario

Allegro, Gianluca

Allen, Andrew

Allen, Bruce G.

Allen, Norman S.

Allen, Sean David

Allwood, Daniel M.

Al-Maharik, Nawaf

Almajano, María Pilar

Almeida Jr., Humberto

Almeida Júnior, José Humberto

Almeida, Adelaide

Almeida-Paes, Rodrigo

Almerico, Anna Maria

Almiro De Almeida, Agostino

Almogi-Hazan, Osnat

Al-Mrabeh, Ahmad

Alonso, Ana P.

Alonso, José L.

Alonso-Moreno, Carlos

Alonso-Vante, Nicolas

Al-Qurayshi, Zaid

Al-Rimawi, Fuad

Alshbool, Fatima Z.

Altamimi, Mohammad

Altamirano-Bustamante, Myriam M.

Altemimi, Ammar

Altieri, Fabio

Altmeppen, Hermann

Altomare, Cosimo

Altucci, Lucia

Alugubelly, Navatha

Aluicio-Sarduy, Eduardo

Alvarado-Lassman, Alejandro

Alvarez Fernandez, Maria Antonia

Alvarez, Alejandra

Alvarez, Ana I.
Álvarez, Eleuterio

Alvarez-Idaboy, Juan Raùl

Alvarez-Parrilla, Emilio

Alvarez-Ramirez, Jose

Álvarez-Suarez, José M.

Alves, Carla Sophia

Alves, Carlos Roberto

Alves, Francisco

Alves, Luis

Alves, Marco

Alves, Wagner A.

Amadio, Emanuele

Amanda, Lumsden

Amano, Tomoko

Amara, Neri

Amaral, Joana S.

Amarowicz, Ryszard

Amato, Felice

Amato, Jussara

Amenabar, Maximiliano

Amer, Bashar

Amer, Hassan

Amero, Carlos

Ami, Diletta

Amigo, Lourdes

Amigo-Benavent, Miryam

Amii, Hideki

Amillis, Sotiris

Amine, Aziz

Amini, Hajar

Aminin, Dmitry

Aminnaji, Morteza

Amiri, Esmaeil

Ammazzalorso, Alessandra

Amo Ochoa, Pilar

Amorati, Riccardo

Amores Arrocha, Antonio

Amorim, Maria Joao

Amoroso, Rosa

Amovilli, Claudio

Amr, Abd El-Galil E.

An, Feifei

An, Hyosung

An, Quanfu

$\mathrm{An}, \mathrm{Yu}$

Ana María, Zamora Martínez

Anand, Jessica P.

Anandhan, Annadurai

Ananieva, Elitsa

Ananthakrishnan, Soundaram Jeevarathinam

Anconi, Cleber 
Ancuceanu, Robert

Anděra, Ladislav

Anderbrant, Olle

Andersen, Pål

Anderson, Harry L.

Andicsová Eckstein, Anita

Andjelkovic, Anuska V.

Andjelkovic, Mirjana

Andolfi, Anna

Andrade Cetto, Adolfo

Andrade, Eugénia De

Andrade, José Carlos

Andrae, Dirk

Andrea, Mahn

Andreana, Peter

Andreasen, Rasmus

Andrei, Sanda

Andreou, Chrysafis

Andreu, Inmaculada

Andreu, Irene

Andreuzzi, Eva

Andrews, Andrew J.

Andrews, David

Andrianov, Alexander

Andrić, Filip

Andruh, Marius

Andrysic, Zdenek

Andrzejewski, Kryspin

Anedda, Roberto

Aneta, Panuszko

Angel, Sergio O.

Angelella, Gina

Angeletti, Mauro

Angeli, Andrea

Angelica De Oliveira, Gomes

Angelici, Gaetano

Angelini, Paola

Angelino, Donato

Angelov, Borislav

Angelov, George

Angelov, Orlin I.

Angelova, Angelina

Angelova, Violina T.

Anghel, Andrei

Angiolella, Letizia

Angius, Fabrizio

Angkawijaya, Artik Elisa

Anibal, Jaime

Aniceto, Natália

Aniello, Francesco

Anis, Badawi
Anisimova, Irina

Anisoara Peptu, Catalina

Anitescu, Cosmin

Anjos, Ofélia

Anni, Marco

Annibal, Andrea

Annor, George

Anraku, Makoto

Ansari, D. Mohd. Azam

Antal, Diana

Antal, István

Anthamatten, Mitchell

Anthemidis, Aristidis

Anticó, Enriqueta

Antoce, Arina Oana

Antoine, Rodolphe

Antolak, Hubert

Anton, Halina

Antoni, Gunnar

Antoniassi, Rosemar

Antoniewska, Agata

Antonino, Famulari

Antônio Casagrande, Gleison

Antonio Malfa, Giuseppe

Antonov, Alexander S.

Antonov, Liudmil

Antunes, Alexandra

Antunes, Dulce

Anugwom, Ikenna

Anzenbacher, Pavel

Aoki, Dan

Aoyagi, Takao

Apetrei, Constantin

Apopei, Andrei Ionut

Appelhans, Dietmar

Appell, Michael

Appendino, Giovanni

Aprea, Eugenio

Aprodu, Iuliana

Aquaro, Stefano

Aquilano, Dino

Aquino, Andrea

Arabski, Michał

Arakawa, Kenji

Arand, Michael

Arango, Rachel A.

Araniciu, Cătălin

Araújo, Evando

Araújo, João M. M.

Araujo, Maria E. M.

Araujo, Pedro 
Araújo, Thaíse Gonçalves

Araya, Samuel Simon

Araya-Maturana, Ramiro

Arce, Pedro F.

Archibald, Steve

Arciello, Angela

Arcone, Rosaria

Arczewska, Marta

Ardévol, Anna

Areche, Carlos

Aremu, Adeyemi Oladapo

Aresta, Antonella

Argentieri, Maria Pia

Arguelles, Sandro

Arias Santiago, Salvador Antonio

Aricò, Fabio

Ariga, Katsuhiko

Arinstein, Arkadi

Arion, Vladimir

Arisawa, Mieko

Armand, Martine

Armanini, Decio

Armenta, Sergio

Armijo, Juan Francisco

Armitage, Bruce

Armitage, Ruth Ann

Arnaboldi, Serena

Arnaouteli, Sofia

Arnason, John

Arnaut, Luís Guilherme

Arnesano, Fabio

Aronica, Laura Antonella

Arora, Neha

Arosio, Paolo

Arrieta, Marina

Arrieta-Baez, Daniel

Arrigo, Rossella

Arrizon, Javier

Arroo, Randolph

Arruebo, Manuel

Arscott, Steve

Arsene, Andreea Letiţia

Arshad, Adeel

Arteaga, Jesús Fernández

Artem'ev, Alexander V.

Artyukhin, Alex

Arugula, Mary Anitha

Arunachalam, Prabhakarn

Arvanitis, Antonios

Aryal, Uma K.

Asadipooya, Kamyar
Asadzadeh, Behnaz

Asahara, Haruyasu

Asakawa, Yoshinori

Asamizu, Shumpei

Asano, Ryutaro

Asaro, Fioretta

Ascheri, Jose

Ascrizzi, Roberta

Asencios, Yvan J. O.

Asfin, Ruslan E.

Ashley, Jon

Ashour, Mohamed Lotfy

Ashpole, Nicole M.

Ashton, Trent

Asín, Laura

Aspatwar, Ashok

Asquith, Christopher R. M.

Assfalg, Michael

Astiasaran, Iciar

Astolfi, Andrea

Ata, Athar

Ata, Seisuke

Ataide, Janaína Artem

Atanasov, Atanas Todorov

Atanasov, Vasil

Atanassova, Maria

Atanassova, Stefka

Atawia, Reem T.

Athanassopoulos, Constantinos

Atobe, Masakazu

Atochin, Dmitriy

Atrián-Blasco, Elena

Attia, Nour F.

Attri, Pankaj

Atuchin, Victor

Aturki, Zeineb

Auberon, Florence

Auffinger, Pascal

Augustyniak, Adrian

Augustyniak, Daria

Auh, Joong-Hyuck

Auladell, Carme

Aullon, Gabriel

Auriemma, Giulia

Ausra, Sipailiene

Au-Yeung, Ho Yu

Avarvari, Narcis

Avato, Pinarosa

Avdeeva, Varvara

Avdonin, Pavel V.

Averin, Alexei D. 
Averina, Elena B.

Avi, Shpigelman

Ávila-Flores, Antonia

Aviles, Francesc Xavier

Avin, Vijay

Aviñó, Anna

Avramescu, Sorin Marius

Avramopoulos, Aggelos

Awate, Sanket

Awni, Rasha A.

Ayala-Zavala, Jesus F.

Azab, Walid

Azad, Mohammad A.

Azaizeh, Hassan

Azhdarinia, Ali

Aziz, Faisal

Azizi, Ali

Azofra, Luis Miguel

Azov, Vlad

Azushima, Kengo

Azzimonti, Barbara

Azzini, Elena

Baars, Johan

Babaev, Eugene

Babafemi, Adewumi John

Babu, Siddharth

Baby, André Rolim

Bacariza, Carmen

Bacciocchi, Michele

Bąchor, Remigiusz

Backhouse, David

Bäckvall, Jan Erling

Bácskay, Ildikó

Bączek, Katarzyna

Baczyńska, Dagmara

Badea, Ildiko

Badea, Mihaela

Badeka, Anastasia

Badimon, Juan J.

Badino, Paola

Badur, Mehmet

Bae, Jung-Woo

Bae, Tae-Hyun

Bae, Youn-Sang

Baeckens, Simon

Baek, Seung-A

Baenas, Nieves

Baeza, Juan José

Bagdžiūnas, Gintautas

Bagetta, Giacinto

Bagi, Péter
Baglioni, Michele

Bagnoli, Luana

Bago, Juli R.

Bagryanskaya, Elena

Bahal, Raman

Bahrim, Gabriela

Bahuguna, Ashutosh

Bai, Chenxi

Bai, Fang

Bai, Lei

Bai, Renren

Baidoo, Edward

Bailly, Christian

Bailly, Fabrice

Bailly, Jean-Denis

Baiocchi, Claudio

Baj, Tomasz

Bajales, Noelia

Bajda, Marek

Bajda, Tomasz

Bajerová, Petra

Bajguz, Andrzej

Bajusz, Dávid

Bak, Andrzej

Bakalkin, Georgy

Baker, Brent A.

Baker, Paul

Baker, Robert

Baki, Gabriella

Bakonyi, Imre

Bakre, Abhijeet A.

Balagurusamy, Nagamani

Balan, Prabhu

Balanč, Bojana

Balaraman, Velmurugan

Balashov, Victor N.

Balasubramanian, Balamuralikrishnan

Balawejder, Maciej

Baláž, Matej

Balažová, Andrea

Balazsi, Csaba

Balcar, Hynek

Balcerczyk, Aneta

Baldassarre, Maria Elisabetta

Baldi, Franco

Baldini, Mario

Baldino, Lucia

Baldisserotto, Anna

Balestra, Dario

Balestri, Francesco

Balewski, Łukasz 
Bálint, Erika

Ballabio, Davide

Ballandras-Colas, Allison

Ballante, Flavio

Ballesté, Ruben Mas

Ballester, Pedro J.

Ballesteros, Menta

Ballesteros-Garrido, Rafael

Balme, Sébastien

Balmer, Lois

Balogh, Krisztián

Balogh, Levente

Balogh, Zsolt

Balslev, Henrik

Balzano, Tiziano

Bamba, Takeshi

Ban, Yusuke

Banach, Marcin

Banach, Mateusz

Banchelli, Martina

Banchero, Mauro

Banciu, Manuela

Bandapalli, Obul Reddy

Bandarenka, Hanna V.

Bandura, Lidia

Bandyopadhyay, Debasish

Bandyopadhyay, Supriyo

Bane, Susan L.

Banerjee, Abhinandan

Banerjee, Debabrata

Bang, Hyoweon

Bang, Marie-Louise

Bankova, Vassya

Banning, Antje

Bannister, Thomas

Banoub, Joseph H.

Bansal, Kuldeep

Banskota, Arjun H.

Banti, Christina N.

Banyasz, Akos

Bao, Jinku

Bao, Yongming

Bao, Yongping

Barakat, Assem

Barałkiewicz, Danuta

Barančík, Miroslav

Baranyai, Edina Fehérné

Barao, Carlos E.

Baratto, Lucia

Barbalexis, Panagiotis

Barbante, Carlo
Barbaric, Monika

Barbasiewicz, Michal

Barbera, Joaquin

Barbero, Nadia

Barbieri, Federica

Barbiero, Isabella

Barbon, Silvia

Barboni, Luciano

Barbosa, Armenio

Barbosa, Marcia

Barbosa, Raquel De Melo

Barbu, Vasilica

Barca, Amilcare

Barceló-Coblijn, Gwendolyn

Bardají, Eduard

Bardet, Michel

Bardi, Giuseppe

Bare, Simon

Barek, Jiří

Barjas, Gustavo Cabrera

Barker, David

Barker, James

Barker, Philip J.

Barker, Tyler

Barki-Harrington, Liza

Barkoulas, Michalis

Barlocco, Daniela

Barlow, James W.

Barnett, David A.

Barok, Márk

Baron, Marco

Baroutaji, Ahmad

Barradas, Thaís Nogueira

Barraja, Paola

Barranco, Angel

Barreca, Davide

Barreca, Salvatore

Barreiro, Maria Filomena

Barreiro, Sonia Losada

Barresi, Antonello

Barreto, Maria Carmo

Barroca, Maria João

Barron, Andrew R.

Barros Gonçalves, Luciana Rocha

Barros, Ana

Barros, Marcelo Paes

Barry, Nicolas

Bar-Shir, Amnon

Bart, Bart Limburg

Bartczak, Piotr

Bartel, Karin 
Bartella, Lucia

Barth, Anders

Bartlett, John

Bartoli, Mattia

Bartolini, Paolo

Bartoloni, Fernando H.

Bartosz, Grzegorz

Bartoszak-Adamska, Elżbieta

Bartoszek, Agnieszka

Bartyzel, Agata

Barvik, Ivan

Barwiolek, Magdalena

Barygina, Victoria

Baryshnikov, Glib

Basavegowda, Nagaraj

Bascands, Jean-Loup

Baschieri, Andrea

Basco, Leonardo

Basile, Adriana

Basile, Teodora

Basini, Giuseppina

Basit, Abdul

Basith, Shaherin

Baskaran, Rathinasamy

Baskin, Igor

Basnett, Pooja

Bassani, Andrea

Bassoli, Angela

Bassols, Anna

Bastarrachea, Luis J.

Bastiat, Guillaume

Bastida, Agatha

Baswan, Sudhir

Baszanowska, Emilia

Batey, Robert T.

Batista, Irineu

Batista, Patrícia

Batista, Ramon

Batista, Ronan

Batta, Gyula

Battaglia, Yuri

Battiato, Alfio

Baud, Stephanie

Baudis, Stefan

Bauer, Eike

Bauer, Rudolf

Baumann, Arnd

Baumann, Heinz

Baumann, Marcus

Baumli, Peter

Bauzá, Antonio
Bavaro, Simona Lucia

Bavaro, Teodora

Bay, Denice C.

Bayer, Peter

Bayer, Wibke

Bayes-Garcia, Laura

Baykov, Sergey

Bayona, Josep

Bayse, Craig

Bazwinsky-Wutschke, Ivonne

Bazylińska, Urszula

Bazylko, Agnieszka

Bazzicalupi, Carla

Beale, David

Beauchemin, Diane

Bębenek, Ewa

Beć, Krzysztof B.

Bechthold, Andreas

Beck, Andreas

Beck, Tyler C.

Beck, William T.

Becker, Daniela

Becker, Guillaume

Becker, Pierre

Beckett, Michael A.

Beckingham, Bryan

Bedini, Emiliano

Bednar, Petr

Bednarek, Radoslaw

Bednárová, Lucie

Bednarski, Patrick J.

Bednarz-Knoll, Natalia

Bedoni, Marzia

Beemon, Karen

Beers, Eric

Beghelli, Daniela

Beghetto, Valentina

Beharry, Andrew

Beharry, Kay D.

Behrends, Volker

Behringer, Erik

Beidaghy Dizaji, Hossein

Beis, Dimitris

Beitz, Toralf

Beklemishev, Mikhail

Bel'skaya, Lyudmila

BelBruno, Joseph

Belcari, Antonio

Belda-Palazón, Borja

Belka, Mariusz

Bell, Thomas W. 
Bellaloui, Nacer

Bellelli, Roberto

Bellemin-Laponnaz, Stéphane

Bellich, Barbara

Bellini, Costanzo

Bello, Claudia

Bello, Martiniano

Belloncle, Christophe

Belloso-Uribe, Kepa

Bellucci, Stefano

Bellumori, Maria

Belluti, Federica

Belmonte, Maria Teresa

Belonenko, Mikhail

Belter, Agnieszka

Beltrán Sanahuja, Ana

Beltran, María Del Carmen Sánchez

Beltrán, Sagrario

Benaglia, Maurizio

Benčić, Đani

Bencini, Andrea

Bencsik, Peter

Bencsik, Timea

Bend, Jack

Bende, Attila

Bender, Dirk

Benedec, Daniela

Benedek, Giorgio

Benediktsdottir, Berglind

Benesperi, Iacopo

Béni, Szabolcs

Beninati, Simone

Benitez, Sonia

Benítez-Mateos, Ana I.

Benito, Adrián

Benito, Juan M.

Bennato, Francesca

Bennis, Noureddine

Benoit, Denizot

Ben-Salem, Salma

Benson, Heather

Bentel, Jacqueline M.

Benvenuti, Stefania

Benyhe, Sandor

Berardi, Anna Concetta

Berdonosov, Peter

Berenjian, Aydin

Beretta, Giovanni L.

Bergamaschi, Matteo

Berger, Daniela Cristina

Berger, Ralf G.
Bergés Tiznado, Magdalena Elizabeth

Berillis, Panagiotis

Berlin, Joshua

Berlin, K. Darrell

Berlowska, Joanna

Berman, Paula

Bermeshev, Maxim

Bermudez, Marcel

Berna, Jose

Bernacchi, Sébastien

Bernal, Vicente

Bernard, Marek K.

Bernardes, Nuno

Bernardi, Luca

Bernardo, Umberto

Bernards, Matthew

Bernascon, Leonardo

Bernat, Przemysław

Bernatchez, Jean

Bernatová, Silvie

Bernède, Jean Christian

Bernhagen, Jürgen

Berni, Roberto

Bernini, Roberta

Bernstein, Elliot

Bernstein, Nirit

Berreau, Lisa M.

Berrotéran-Infante, Neydher

Berrue, Fabrice

Berski, Wiktor

Berteina-Raboin, Sabine

Bertelloni, Fabrizio

Berthold-Pluta, Anna

Berti, Federico

Bertin, Matthew J.

Bertrand, Martine

Bertsch, Arnaud

Bertsch, Pascal

Bertsouklis, Konstantinos

Bertuzzi, Giulio

Bertuzzi, Terenzio

Besalú, Emili

Bessmertnykh-Lemeune, Alla

Besson, Thierry

Best, Stephen

Betancur, Stefanía

Betekhtin, Alexander

Betemariam, Senait Ashenafi

Betoret, Noelia

Bettencourt, Ana Francisca

Bettini, Simona 
Bettotti, Paolo

Beyeh, Ngong Kodiah

Beyer, Sebastian

Bezirtzoglou, Eugenia

Bhandari, Dhaka Ram

Bharadwaj, Shiv

Bharath, Leena P.

Bhargava, Aditi

Bhaskar, Abhinav

Bhat, Owais

Bhatt, Priyanka

Bhattacharjee, Apurba Krishna

Bhattacharjee, Sonali

Bhattacharya, Abhisek

Bhattacharya, Pratip

Bhattacharya, Rajat

Bhattacharya, Supriyo

Bhatti, Muhammad Mubashir

Bhosale, Sheshanath

Bhowmik, Pradip K.

Bhusal, Ram

Biagi, Marco

Bialecka-Florjańczyk, Ewa

Białek, Agnieszka

Białek, Małgorzata

Bian, Huiyang

Bian, Yongzhong

Bianchi, Antonio

Bianchi, Massimiliano

Bianchini, Francesca

Bianco, Andrea

Bianco, Armandodoriano

Bianco, Giuliana

Biancolillo, Alessandra

Bidarra, Sílvia J.

Bideshi, Dennis

Bidet, Yannick

Bidwai, Ashok

Biedermann, David

Biel, Wioletta

Bielas, Rafał

Bielejewski, Michal

Bielenica, Anna

Bieliński, Dariusz M.

Biernasiuk, Anna

Biesaga, Magdalega

Biffis, Andrea

Bijak, Michał

Bilal, Muhammad

Bilancio, Antonio

Bildstein, Benno
Bílek, Vlastimil

Bilewicz, Aleksander

Bilia, Anna Rita

Bilitewski, Ursula

Billard, Thierry

Bilska-Wilkosz, Anna

Bin, $\mathrm{Xu}$

Binda, Elisa

Bini, Roberto

Biniari, Katerina

Binotto, Gianni

Biondi, Marco

Biot, Christophe

Birch, Brian

Bird, Phillip I.

Birke, Ronald L.

Birková, Anna

Birsa, Mihail Lucian

Bisbey, Ryan

Bisceglie, Franco

Bischof, Johannes

Bishop, Roger

Bisio, Francesco

Bišová, Kateřina

Bisti, Silvia

Bis-Wencel, Hanna

Bisz, Elwira

Biziuk, Marek

Biziuk, Marek K.

Bizzarri, Bruno Mattia

Bizzarri, Mariano

Bizzo, Humberto

Bjerklin, Krister

Bjornstedt, Mikael

Blacha-Grzechnik, Agata

Black, David

Blackwell, Barbara

Blagojevic Zagorac, Gordana

Blakemore, James D.

Blalock, William

Blamires, Sean

Blancaflort, Lluís

Blanco, Angeles

Blanco, Eduardo

Blanco, Francisco J.

Blanco-Fernandez, Barbara

Blandin, Gaetan

Blando, Federica

Blanton, Cynthia

Blasi, Francesca

Blasiak, Janusz 
Błaszczyk, Jarosław

Blay, Gonzalo

Blay, Vincent

Blazevic, Ivica

Błażewicz, Anna

Błażewska, Katarzyna M.

Blazquez, Ana-Belen

Blázquez, Enrique

Blersch, David M.

Blind, Raymond

Blindauer, Claudia A.

Bloch, Sylwia

Bloch, Witold

Blom, Burgert

Blonska, Marzenna

Blough, Bruce E.

Blumel, Janet

Blythe, Linda L.

Boanini, Elisa

Bobak, Łukasz

Bobek, Jan

Bober-Majnusz, Katarzyna

Bobis, Otilia

Bobrovsky, Alexey

Bocarsly, Miriam

Bochkarev, M. N.

Boddaert, Thomas

Bode, Bela Ernest

Bodea, Liviu-Gabriel

Bodenstine, Thomas

Bodoki, Ede

Boege, Fritz

Boeglin, Alex

Boehr, David D.

Boekema, Bouke

Boese, Adrian Daniel

Boffo, Elisangela F.

Boga, Carla

Bogdan, Catalina

Bogdanchikova, Nina

Bogdanov, Alexey A.

Bogdański, Paweł

Boggia, Raffaella

Boggioni, Laura

Bogliotti, Nicolas

Bognár, Gabriella

Bogush, Anna

Bogusławska, Joanna

Bohinc, Klemen

Böhm, Volker

Bohman, Björn
Boichuk, Sergei

Boika, Aliaksei

Bojarová, Pavla

Bojarska, Joanna

Bojić, Mirza

Bojková, Bianka

Bokach, Nadezhda

Boland, Mike

Bolanos-Garcia, Victor M.

Bölcskei, Hedvig

Bölcskei, Kata

Boldizsár, Ferenc

Bolibruchová, Dana

Bolokang, Amogelang Sylvester

Bolonio, David

Bolotin, Dmitrii S.

Bonacina, Fabrizia

Bonadies, Irene

Bonamore, Alessandra

Bonardd, Sebastian

Bonasera, Aurelio

Bonaventura, Giovanni

Bondon, Arnaud

Bondzior, Bartosz

Bonferoni, Maria Cristina

Bongarzone, Italia

Bonikowski, Radosław

Bonin, Serena

Bonnet, Célia S.

Bonnet, Pascal

Bonnet, Pierre-Antoine

Bonofiglio, Daniela

Bonomo, Matteo

Bonrath, Werner

Bontempo, Paola

Bonucci, Alessio

Bonyár, Attila

Boo, Yong Chool

Booth, Brian

Boratyński, Przemysłąw

Borbás, Anikó

Borbas, K. Eszter

Borcan, Florin

Bordignon, Enrica

Borek, Wojciech

Borges, Alisson

Borges, Ricardo

Borges, Warley

Borghi, Monica

Borgonovo, Gigliola

Boris, Pejin 
Bork, Kaya

Borka, Duško

Borkowska, Edyta Marta

Borole, Abhijeet

Boroń, Dariusz

Boros, Danuta

Boros, László

Borota, Ana

Borowiec, Joanna

Borowik, Agata

Borse, Vikrant

Borska, Sylwia

Bortolozzi, Roberta

Bortoluzzi, Marco

Borys, Krzysztof

Boryski, Jerzy

Borza, Tudor

Bosch, Joan

Boschi-Muller, Sandrine

Boschin, Giovanna

Boscoboinik, Jorge Anibal

Boselli, Emanuele

Bosetti, Michela

Boskovic, Zarko

Bosnar, Maja Herak

Bosso, Andrea

Boström, Kristina Bengtsson

Botana, Ana María

Botelho, João

Botez, Elisabeta

Botha, Frederik

Botondi, Rinaldo

Botoran, Oana

Botta, Bruno

Bottari, Fabio

Botubol-Ares, José Manuel

Bou, Ricard

Boucekkine, Abdou

Boucheix, Claude

Boucher, Dave

Boucher, David S.

Bouckaert, Julie

Boudaden, Jamila

Boudon, Julien

Bougrin, Khalid

Bouillaud, Frederic

Boukouvalas, John

Bourdon, Emmanuel

Bourgoin, Sylvain

Bourneuf, Emmanuelle

Bouropoulos, Nikolaos
Bourson, Patrice

Bouvier, Benjamin

Bovi Karatay, Graziele Grossi

Bowden, Bruce

Bowman-James, Kristin

Boyle, Maria

Boysen, Reinhard

Bozdag, Murat

Bozio, Renato

Bozzo, Giancarlo

Brabet, Philippe

Bracher, Franz

Bradley, Mark

Bradshaw, Ben

Bradshaw, Patrick

Braendle, Christian

Braga, Anna Rafaela Cavalcante

Braga, Maria-Helena

Braga, Susana Santos

Braicu, Cornelia

Braig, Simone

Bramanti, Emilia

Branca, Ferdinando

Branca, Jacopo Junio Valerio

Branca, Rosa Tamara

Brancart, Joost

Brancato, Virginia

Brandt, Curtis

Brás, Natércia

Brasic, James

Braslavsky, Silvia E.

Brassat, Katharina

Bratu, Ana

Braun, Andrew P.

Braunstein, Pierre

Bravo, Lidia

Bravo, Susana B.

Brayden, David J.

Brčić Karačonji, Irena

Brea, Oriana

Brebu, Mihai

Brecker, Lothar

Breeze, Alex

Bregadze, Vladimir

Brégier, Frédérique

Brehm, Maria Alexandra

Brehm, Martin

Breidbach, Andreas

Breinbauer, Rolf

Brela, Mateusz

Bren, Urban 
Brender, Jeffrey

Bresciani, Elena

Brestic, Marian

Breton, Gary W.

Breugst, Martin

Breza, Martin

Brezesinski, Torsten

Brezovský, Jan

Brichacek, Matthew

Briedis, Vitalis

Briffa, Sophie

Briganti, Matteo

Brígido, Clarisse

Brignole, Chiara

Brindisi, Matteo

Brindley, David

Britton, Jonathan

Briz, Oscar

Broadhurst, Catherine Leigh

Brocławik, Ewa

Broda, Małgorzata A.

Brodosi, Lucia

Brogi, Simone

Broniatowski, Marcin

Bronisz, Robert

Bronowska, Agnieszka

Brook, David

Brooks, Allen

Brooks, Benjamin D.

Brotin, Thierry

Brouwers, Jos F.

Brouzgou, Angeliki

Brovarets, Volodymyr S.

Brown, Alan B.

Brown, Dennis

Brown, Janet E.

Brown, Lindsay

Brown, Tom

Brozovic, Anamaria

Bruce, James

Bruckner, Christian

Bruder-Nascimento, Thiago Do

Brugarolas, Pedro

Bruijn, Johannes De

Brulíková, Lucie

Brullo, Chiara

Brune, Wolfram

Brunel, Fabrice

Brunetti, Luigi

Brünnert, Daniela

Bruno, Mattos
Bruno, Victor

Bruns, Carson J.

Brunschweiger, Andreas

Brüssow, Harald

Brust, Tarsis

Brust-Mascher, Ingrid

Brutti, Sergio

Bryant, Matthew S.

Brycki, Bogumil

Bryjak, Marek

Bryk, Paweł

Bryla, David

Brys, Joanna

Brzeska, Joanna

Brzeszcz, Joanna

Brzezinski, Marek

Brzozowski, Tomasz

$\mathrm{Bu}$, Pengli

Bubnov, Alexey

Buchberger, Wolfgang

Buchet, Rene

Buchowicz, Włodzimierz

Buchtová, Hana

Buckley, M.

Bucur, Bogdan

Budryn, Grażyna

Budyka, Mikhail F.

Budzak, Simon

Budzevich, Mikalai

Budzevich, Mikalai M.

Budzianowski, Jaromir

Budzisz, Elżbieta

Bueno-Silva, Bruno

Bugarin, Alejandro

Bugni, Tim

Buha, Aleksandra

Buica, Astrid

Buijs, Wim

Bujak, Maciej

Bujak, Tomasz

Bujanovic, Biljana

Bujnicki, Bogdan

Bukowski, Michal

Bulánek, Roman

Bulatov, Emil

Bulboacă, Adriana Elena

Bulet, Philippe

Bulgariu, Laura

Bülow, Margret $\mathrm{H}$.

Bulushev, Dmitri

Buma, Wybren J. 
Bun Ng, Tzi

Bunce, Richard A.

Bunea, Andrea

Bungau, Simona

Bungau, Simona Gabriela

Buniowska, Magdalena

Buonocore, Francesco

Bura-Nakić, Elvira

Buranelo Egea, Mariana

Burcul, Franko

Burdak-Rothkamm, Susanne

Bureau, Ronan

Bures, Filip

Burge, Kathryn

Burgos, Rafael

Burgos-Ramos, Emma

Burke, Anthony

Burket, Jessica

Burkin, Maxim

Burley, Jonathan C.

Burns, Jonathan R.

Burns, Jorge $S$.

Burpo, Fred

Burrai, Giovanni Pietro

Burrola-Aguilar, Cristina

Bursic, Vojislava

Burton, Zachary F.

Bury, Wojciech

Busardò, Francesco Paolo

Busby, Chris

Busco, Giovanni

Businaro, Rita

Busquets, Maria Antònia

Busto, Natalia

Bustos, Diego Martin

Buszewski, Bogusław

Butenschön, Holger

Butler, Andrew A.

Butler, John

Buzuk, Marijo

Buzzá, Hilde Harb

Bydlowski, Sérgio Paulo

Bylund, Dan

Byrdin, Martin

Byrne, Hugh

Bystrom, Jonas

Byun, Sanguine

Caballero, Antonio

Caballero, Daniel

Caballero-Casero, Noelia

Caballero-Garcia, Beatriz
Cabello-Garcia, Tomás

Cabeza Gras, Óscar

Cabral, Horacio

Cabrales, Luis

Cabrele, Chiara

Cabrera, Humberto

Cabrera-Chávez, Francisco

Cabrera-Fuentes, Hector A.

Caccia, Mario

Cacciola, Francesco

Cáceres, Alejandro

Cachada, Anabela Ferreira De Oliveira

Cacini, Sonia

Caddeo, Carla

Cadiou, Cyril

Cádiz-Gurrea, María De La Luz

Cafforio, Paola

Cafiero, Mauricio

Caggia, Silvia

Cagliero, Cecilia Lucia

Cahlíková, Lucie

Cai, Bishuang

Cai, Demin

Cai, Lisheng

Cai, Weimin

Cai, Wenlong

Cai, Zhengxu

Caille, Fabien

Cailliau, Katia

Cailloux, Jonathan

Caires, Hugo R.

Cairns, Warren Raymond Lee

Cairrão, Elisa

Čakić Semenčić, Mojca

Cala, Antonio

Calabrese, Luigi

Calabretta, Maria Maddalena

Calabrò, Paolo S.

Calapai, Gioacchino

Calasso, Maria

Calatayud, Paul-andré

Calcabrini, Cinzia

Calcaterra, Andrea

Calcinotto, Arianna

Calcutt, Michael

Calderisi, Marco

Calderón De La Barca, Ana María

Caleb, Oluwafemi James

Calhelha, Ricardo Costa

Calì, Tito

Caliceti, Cristiana 
Califano, Valeria

Caligiuri, Isabella

Calignano, Antonio

Calina, Daniela

Calinescu, Ioan

Calín-Sánchez, Ángel

Calissendorff, Jan

Callery, Patrick S.

Calligari, Paolo

Callone, Emanuela

Caloca, Jose Maria

Calogeropoulou, Theodora

Calokerinos, Antony C.

Calpena, Ana C.

Caltagirone, Claudia

Calucci, Lucia

Calvano, Cosima D.

Calvano, Cosima Damiana

Calvo, Conceptión

Calvo, Lourdes

Calvo, Marta M.

Calzaferri, Gion

Camacho, M. Encarnación

Camacho-Corona, María Del Rayo

Camaiti, Mara

Camara, Jose Sousa

Camarasa, María-José

Camba, A. Tundidor

Camele, Ippolito

Cameron, Donald

Cameron, Simon

Caminade, Anne-Marie

Camins, Antoni

Camire, Mary

Campagne, Jean-Marc

Campana, Raffaella

Campanella, Alessandro

Campanella, Beatrice

Campbell, Bradley

Campbell, Colin

Campbell, Grant R.

Campbell, Kris

Campbell, Phil G.

Campiglia, Pietro

Campilho, Ana

Campillo, Natalia

Campo, Giuseppe

Campo, Riccardo

Campone, Luca

Campos Rosa, Joaquín María

Campos, Alex Fabiano Cortez
Campos, Maria

Campos, Rafael Kroon

Campos-Martin, Jose M.

Campos-Martínez, José

Camps, Ihosvany

Canas, Sara

Cancemi, Patrizia

Candeias, Nuno R.

Candiani, Gabriele

Canela, Núria

Canela-Garayoa, Ramon

Caneschi, Andrea

Canetta, Elisabetta

Canfield, Scott

Canini, Antonella

Cannistraro, Salvatore

Cannon, Richard

Canonico, Barbara

Cantamessa, Simone

Cantarelli, Miguel

Cantini, Francesca

Canuti, Valentina

Cao, Deliang

Cao, Jian

Cao, Jianyun

Cao, Xudong

Cao, Yongsong

Capasso, Francesco

Capasso, Ilaria

Capasso, Raffaele

Capillo, Gioele

Capogni, Marco

Caponio, Francesco

Capozzi, Vittorio

Cappellacci, Loredana

Capperucci, Antonella

Cappiello, Mario

Capriati, Vito

Capuano, Federico

Capurso, Giovanni

Caputo, Fanny

Caputo, Gregory

Caputo, Paolino

Caraballo, Mauricio

Carabelli, Valentina

Carabineiro, Sonia

Carac, Geta

Carafa, Maria

Carata, Elisabetta

Carazo, Alejandro

Carballeira, Nèstor M. 
Carbonaro, Carlo Maria

Carbone, Alessandra

Carcadea, Elena

Carcelli, Mauro

Carda, Miguel

Cardador-Martínez, Anaberta

Cardellicchio, Cosimo

Cardenas Zuniga, Roberto

Cardia, Maria Cristina

Cardona, Francesca

Cardoso, Susana

Cardoso, Susana M.

Carfora, Anna

Carignani, Elisa

Carina, Valeria

Cariou, Kevin

Carla Aragoni, Maria

Carlberg, Carsten

Carlier, Paul

Carlini, Célia R.

Carlino, Elvio

Carlos Salay, Luiz

Carlotto, Silvia

Carmen, Mejía

Carmona, Estefanía Núñez

Carmona-Ribeiro, Ana M.

Carnevale Neto, Fausto

Carney, Randy

Carniato, Fabio

Caroleo, Maria Cristina

Caronni, Sarah

Carosio, Federico

Carpanese, Debora

Carpentieri, Andrea

Carpes, Solange Teresinha

Carqueijeiro, Inês

Carr, Carolyn A.

Carradori, Simone

Carrasco, Héctor

Carrasco, Sergio

Carrasco-López, César

Carré, Vincent

Carregal-Romero, Susana

Carrella, Luca

Carrera Fernández, Ceferino

Carrera, Mónica

Carrero, Jose Antonio

Carretto, Edoardo

Carriero, Maria Vincenza

Carrillo, Celia

Carrillo, Wilman
Carrión, Mar

Carrubba, Alessandra

Carter, Katharine

Carter, Wayne

Carullo, Gabriele

Caruntu, Constantin

Caruso, Calogero

Caruso, Enrico

Caruso, Giuseppe

Caruso, Ugo

Carvajal-Millan, Elizabeth

Carvalheiro, Manuela Colla

Carvalho Pimenta, Daniel

Carvalho, Daniel

Carvalho, Luisa M.H.

Casadio, Rita

Casado-Coterillo, Clara

Casado-Martinez, M. Carmen

Casampai, Antal

Casanova, Llanos

Casasnovas, Rodrigo

Casciaro, Bruno

Cascone, Sara

Case, Raymundo

Cásedas, Guillermo

Casey, Jennifer

Casiello, Michele

Casiraghi, Antonella

Casnati, Alessandro

Casoni, Dorina

Cassidy, John F.

Castagna, Michela

Castaldo, Rachele

Castañeda-Corral, Gabriela

Castan-Laurell, Isabelle

Castellano, Carlo

Castellari, Massimo

Castelli, Ivano E.

Castelli, M. Paola

Castellini, Elena

Castellón, Enrique Rodríguez

Castelo-Branco, Miguel

Castiglione, Franca

Castilho, Paula

Castillo, Antonio

Castillo, Francisca

Castillo, Juan

Castillo, Sandra

Castñeiras, Alfonso

Castoldi, Lidia

Castorena-Torres, Fabiola 
Castro, Eulogio

Castro, José Javier Fernández

Castro, Mariana S.

Castro, Remedios

Castro, Ricardo A.E.

Castro-Vargas, Henry I.

Castrucci, Ana Maria

Cataldi, Tommaso

Cataldo, Salvatore

Catani, Martina

Catanzaro, Giuseppina

Catapano, Carlo

Catar, Rusan

Catarino, Isabel

Catarino, Marcelo D.

Catarino, Susana

Catauro, Michelina

Caterino, Marianna

Cattoën, Xavier

Catucci, Gianluca

Catucci, Lucia

Catuogno, Silvia

Cauchy, Thomas

Caucia, Franca

Cauda, Valentina

Cautela, Domenico

Cauteruccio, Silvia

Cava, Claudia

Cavalcanti, João Henrique F.

Cavaleiro, José A. S.

Cavallaro, Giuseppe

Cavallo, Carmen

Cavalluzzi, Maria Maddalena

Cavani, Luciano

Cavazzini, Alberto

Cavé, Christian

Cavicchi, Kevin

Caviglia, Gian Paolo

Caydamli, Yavuz

Cazacu, Maria

Cazzola, Roberta

Cebecauer, Marek

Ceccarelli, Gabriele

Ceccato-Antonini, Sandra Regina

Cecilia, Juan Antonio

Cecioni, Samy

Cefalas, Alciviadis-Constantinos

Celakovska, Jarmila

Celano, Rita

Celeiro, María

Celetti, Angela
Cella, Eleonora

Cellamare, Saverio

Cembrowska-Lech, Danuta

Cena, Cícero

Čènas, Narimantas

Cendrowski, Andrzej

Cepeda, Javier

Cerazy-Waliszewska, Joanna

Cerbo, Alessandro Di

Cerezo, Javier

Černáková, Lucia

Černý, Jiří

Cerny, Miroslav

Cerón-Carrasco, José Pedro

Cerra, Bruno

Cerrato, Giuseppina Pinuccia

Cerri, Guido

Cerri, Marcel Otavio

Cerrito, Maria Grazia

Cerrone, Federico

Cerruti, Pierfrancesco

Cerveny, Silvina

Cesar, Vincent

Cesarino, Igor

Cesaro, Attilio

Cescutti, Paola

Česonienè, Laima

Ceulemans, Arnout

Cézard, Christine

Chabaud, Laurent

Chacko, Jenu Varghese

Chae, Michael

Chai, Kyu Yun

Chai, Tsun-Thai

Chaimbault, Patrick

Chakrabarti, Subhadeep

Chakraborty, Anutosh

Chakraborty, Atanu

Chakraborty, Joydeep

Chalashkanov, Nikola

Chaleckis, Romanas

Chalioris, Constantin

Chalupka, Karolina

Chamcheu, Jean Christopher

Chami, Goylette

Champagne, Benoît

Champagne, Pier Alexandre

Champouret, Yohan

Chan, Ben C. L.

Chan, Catherine

Chan, Chi On 
Chan, Yin-Ching

Chan, Yi-Tsu

Chan, Yu-Yi

Chandra, Boggarapu Praphulla

Chandramouli, Kulshreshtha

Chandrasekaran, Murugesan

Chandrasekaran, Srinivas Niranj

Chandru, Kuhan

Chandrudu, Saranya

Chang, Chang-Tang

Chang, Che-Chien

Chang, Cheng-Chung

Chang, Cheng-Wei Tom

Chang, Chia Ming

Chang, Chia-che

Chang, Chien-Chung

Chang, Chih-Wei

Chang, Ching-yao

Chang, Chuang-Rung

Chang, Geng-Ruei

Chang, Hai-Chou

Chang, Hsueh-Wei

Chang, Hsun-Shuo

Chang, Jui-Cheng

Chang, Kun-Che

Chang, Kunok

Chang, Long-Sen

Chang, Sue-Joan

Chang, Te-Sheng

Chang, Wei-Jen

Chang, Wen-Wei

Chang, Yuan Shiun

Chang, Yu-Wei

Chao, Chih-Hua

Chao, Louis Kuoping

Chapman, Nadine

Chaptal, Vincent

Charest, Pascale

Charitidis, Costas A.

Charmas, Barbara

Chartier, Agnes

Chartoumpekis, Dionysios

Chassande, Olivier

Chatel, Gregory

Chatgilialoglu, Chryssostomos

Chatterjea, Devanani

Chatterjee, Abhijit

Chatterjee, Ruchira

Chatterjee, Sudipta

Chatzaki, Ekaterini

Chatzidoukas, Christos
Chatzifragkou, Afrodoti

Chaud, Marco

Chauhan, Neeraj

Chaurasia, Akhilesh Kumar

Chava, Rama Krishna

Chaves, Sílvia

Chavez, Carlos A. Sanhueza

Chavez, Ferman

Chavez-Calvillo, Gabriela

Chavez-Santoscoy, Rocio Alejandra

Chawla, Aseem

Checconi, Paola

Chechik, Victor

Cheel, José

Chekmenev, Eduard

Chellan, Nireshni

Chelli, Riccardo

Chemat, Farid

Chen, Bingdi

Chen, Binling

Chen, Bolin

Chen, Charles

Chen, Chau-Chyun

Chen, Chi

Chen, Chi-Tien

Chen, Chuan-Lin

Chen, Chung-Hwan

Chen, Chun-Jung

Chen, Chun-Lin

Chen, Gang

Chen, Guangping

Chen, Guoxun

Chen, Haixia

Chen, Hao

Chen, Hao Ping

Chen, Haoqing

Chen, Hongzhang

Chen, Hsin-Chun

Chen, Hsing-Yin

Chen, Hsuan-Ying

Chen, Hung-Yu

Chen, James K

Chen, Jeff Yi-fu

Chen, Jen-Tsung

Chen, Jhy-Der

Chen, Jiahao

Chen, Jianbo

Chen, Jing-Hsien

Chen, Jun

Chen, Kaihong

Chen, Kelvin H.-C. 
Chen, Li

Chen, Liang-Yu

Chen, Lih-Geeng

Chen, Lili

Chen, Lin

Chen, Lingxin

Chen, Mei-Chuan

Chen, Mingli

Chen, Mingsheng

Chen, Mingzhou

Chen, Minjian

Chen, Mu-Kuan

Chen, Po-Wen

Chen, Qi

Chen, Qiao-Hong

Chen, Qixian

Chen, Shiu-Nan

Chen, Tao-Hsing

Chen, Wei

Chen, Wei-Ting

Chen, Wen-Ying

Chen, Xiao-Lan

Chen, Xiaoxin

Chen, Xin

Chen, Xinjian

Chen, Xiujuan

Chen, Xue-Gang

Chen, Yeh

Chen, Yen-Hau

Chen, Yi-Fan

Chen, Yijia

Chen, $\mathrm{Yu}$

Chen, Yung-Chung

Chen, Yun-Ju

Chen, Zhihang

Chen, Zhijun

Chen, Zhongning

Chénais, Benoit

Cheng, Chih-Chia

Cheng, Fong-Yu

Cheng, Gang

Cheng, Juei-Tang

Cheng, Jya-Wei

Cheng, Pei

Cheng, Qingqing

Cheng, Qunkang

Cheng, Sen-Sung

Cheng, Tao

Cheng, Xiaodong

Cheng, Xingguo

Cheng, Xinlai
Cheng, Yi-Sheng

Cheng, Yuan-Bin

Cheng, Yu-Jung

Cheon, Dong-Joo

Cheong, Yuen-Ki

Cheraghi Bidsorkhi, Hossein

Cheraghian, Goshtasp

Cheret, Jérémy

Cherkaoui Malki, Mustapha

Chermette, Henry

Chernikov, Oleg

Chernova, Tatiana

Cheshenko, Natalia

Chetcuti, Michael J.

Chetwynd, Andy

Cheynier, Véronique

Chi, Gerald

Chianese, Giuseppina

Chiang, Anthony S. T.

Chiang, Chien-Min

Chiang, Chi-Ling

Chiang, Hsiu-Mei

Chiang, Wen-Dee

Chiang, Ya-Yu

Chiarella, Emanuela

Chiarelli, Laurent

Chiarini, Marco

Chiavaroli, Annalisa

Chichorro, Juliana G.

Chien, Hsiu-Wen

Chien, Shih-Chang

Chien, Yi-wen

Chifiriuc, Carmen

Chifiruc, Mariana Carmen

Chilibeck, Phil

Chilton, Lisa

Chimenti, Isotta

Chimienti, Guglielmina Alessandra

Chin, Young-won

Chinchilla, Rafael

Chinnathambi, Shanmugavel

Chiou, Chun-Tang

Chirgwin, John M.

Chiriac, Aurica

Chirila, Traian

Chis, Vasile

Chisaka, Mitsuharu

Chisholm, John D.

Chistyakov, Vladimir Anatolievich

Chisu, Valentina

Chitchumroonchokchai, Chureeporn 
Chitescu, Carmen Lidia

Chitgupi, Upendra

Chittori, Sagar

Chiu, Chih-Wei

Chiu, Ching-Feng

Chiummiento, Lucia

Chizhov, Alexander O.

Chizzola, Remigius

Chlichlia, Katerina

Chmielarz, Lucjan

Chmielarz, Paweł

Chmielewski, Tomasz

Cho, Dae Won

Cho, Deok-Yong

Cho, Dong-Woo

Cho, Hea-Young

Cho, Hyun-Jong

Cho, Jae Youl

Cho, Sang Wan

Cho, Sunghun

Cho, Yi Tzu

Chobot, Vladimír

Chocholouš, Petr

Choi, Hyukjae

Choi, Hyung-Kyoon

Choi, Insung

Choi, Jae-Hong

Choi, Jane Ru

Choi, Jeong-Woo

Choi, Jonghoon

Choi, Jongmin

Choi, Nag Jung

Choi, Seungkeun

Choi, Tae Gyu

Choi, Young Hae

Choi, Young Hee

Choi, Young Whan

Chojnacki, Jerzy

Cholewinski, Grzegorz

Choquesillo-Lazarte, Duane

Chorazy, Szymon

Chorna, Nataliya

Choromanska, Anna

Choshi, Tominari

Chou, C. James

Chou, Weichun

Chouhan, Raghuraj Singh

Chow, James C. L.

Chow, Simon Kwoon-Ho

Chowdhury, Abu Faem Mohammad Almas

Chowdhury, Pankaj
Chowdhury, Ratul

Chrcanovic, Bruno

Chreptowicz, Karolina

Christensen, Jørn B.

Christensen, Lars P.

Christina, Barja-Fidalgo

Christodoulou, Maria-Ioanna

Christoffer Eklund, Patrik

Chrobak, Elwira

Chronakis, Nikos

Chronopoulou, Evangelia

Chronopoulou, Laura

Chruszcz, Maksymilian

Chrysargyris, Antonios

Chrzanowski, Łukasz

Chu, Chun Hung

$\mathrm{Chu}$, Pei-Ming

$\mathrm{Chu}$, Xiangping

Chuang, Er-Yuan

Chubarov, Alexey

Chueh, Anderly C.

Chueh, Pin Ju

Chun, Jong Tai

Chun, Kyung-Soo

Chung, Ching-Hu

Chung, Dong Young

Chung, Doo Soo

Chung, Gehoon

Chung, Sheng-Heng

Chung, Suk-Jae

Chung, Yeonseok

Churio, Maria

Chuyen, Hoang V.

Chwalek, Karolina

Chwalibog, André

Chworos, Arkadiusz

Chyau, Charng-Cherng

Chylińska, Marta

Ciampi, Simone

Ciancaleoni, Gianluca

Ciancetta, Antonella

Cianfaglione, Kevin

Ciardelli, Francesco

Ciardiello, Maria Antonietta

Ciarlo, Laura

Ciasca, Gabriele

Ciccarelli, Renata

Cicchi, Stefano

Cicco, Nunzia

Ciccone, Marco

Çiçek, Serhat Sezai 
Cicenas, Jonas

Cicero, Arrigo

Cichero, Elena

Cielecka-Piontek, Judyta

Ciesielski, Wojciech

Cieśla, Jolanta

Cieslar, Grzegorz

Cieslik, Wioleta

Cifone, Maria Grazia

Cilla, Antonio

Cillo, Fabrizio

Cimas, Francisco J.

Cimino, Roberto

Cimpoiu, Claudia

Cinelli, Patrizia

Cinteza, Otilia Ludmila

Ciobica, Alin

Ciogli, Alessia

Ciolacu, Diana

Ciornea, Elena Todirascu

Čipak Gašparović, Ana

Cipak, Lubos

Cipakova, Ingrid

Cipullo, Roberta

Circiumaru, Adrian

Circu, Viorel

Cirillo, Emilia

Cirillo, Giuseppe

Cirmi, Santa

Cirri, Damiano

Cirrincione, Girolamo

Cirtoaje, Cristina

Cirujano, Francisco

Ciszewski, Wojciech

Citores, Lucia

Ciufolini, Marco A.

Čižmár, Erik

Čizmić, Mirta

Claramunt, Rosa M.

Claridge, Jolyon K.

Clark, Benjamin

Clark, Georgina

Clarke, Anthony J.

Clarke, William Alex

Claudia, Delgadillo Puga

Claudio, Trapella

Claus, Harald

Clavijo-McCormick, Andrea

Clegg, Jack K.

Clément, Gilles

Clement, Yuen
Clemetson, Kenneth J.

Clericuzio, Marco

Coates, Dawn

Cobb, Steven L.

Cocco, Lucio

Cocero Alonso, María José

Cocklin, Simon

Cocovi Solberg, David Jaime

Coderch, Claire

Codina, Georgiana

Coelho-Silva, Juan

Coetzer, Theresa

Cohen, Boiko

Cohen, Yachin

Cojocaru, Corneliu

Colangelo, Anna Maria

Colavito, Sierra A.

Colby, David

Coldea, Teodora Emilia

Colina-Márquez, Jose

Coll, Josep

Collet, Trudi

Collina, Simona

Collinge, David B.

Colomban, Philipe

Colombo, Diego

Colonna, Bianca

Colonna, Giovanni

Čolović, Mirjana B.

Coltelli, Maria Beatrice

Coman, Simona M.

Coman, Vasile

Comas-Garcia, Mauricio

Combès, Audrey

Comfort, Kristen K.

Comito, Robert J.

Commisso, Mauro

Comparat, Daniel

Conan, Francoise

Concellon, Alberto

Concilio, Simona

Conconi, Maria Teresa

Condelli, Nicola

Condello, Maria

Condorelli, Guglielmo Guido

Conejero, Salvador

Conforti, Filomena

CongBao, Kang

Connor, Mark

Conran, John

Constantin, Lucian 
Constantinescu-Aruxandei, Diana

Constantinides, Christos

Constantinou, Violetta

Consumi, Marco

Contardi, Marco

Contel, Maria

Conterosito, Eleonora

Conti, Claudia

Conti, Pio

Contiero, Jonas

Contreras-Cornejo, Hexon Angel

Coombs, Melanie

Cooper, Robin

Copeland, Les

Copolovici, Dana Maria

Copolovici, Lucian

Coppedè, Fabio

Coradin, Thibaud

Corallo, Claudio

Corchado, Jose C.

Cordaro, Marika

Cordeiro, Ana Sara

Cordero, Chiara Emilia

Cordero-Lanzac, Tomas

Córdoba, Manuel

Córdoba-Diaz, Manuel

Corke, Harold

Cornea-Cipcigan, Mihaiela

Cornejo, Alberto

Cornelis, Marilyn

Cornett, Claus

Cornu, Agnes

Coroaba, Adina

Corradini, Valdis

Correa Pacheco, Zormy Nacary

Correa, Arkaitz

Corrêa, Arlene Gonçalves

Corrêa, Camila Renata

Correa, Diego Rafael Nespeque

Correcher, Virgilio

Correia, Ana Cristina

Correia, João D. G.

Correia, Manuela

Correia-de-Sá, Paulo

Corsaro, Carmelo

Corsaro, Maria Michela

Corsi, Ilaria

Corte, Laura

Cortés, Cristóbal

Cortés, Farid B.

Cortiella, Mariona Gil
Cortijo, Miguel

Corvis, Yohann

Corvo, Marta C.

Corzana, Francisco

Cosco, Donato

Coscueta, Ezequiel

Cosenza, Gianfranco

Cosimbescu, Lelia

Coskun, Ahmet F.

Cosma, Pinalysa

Cosme, Fernanda

Cossairt, Brandi M.

Cossignani, Lina

Cossio, Fernando

Costa, Ana

Costa, Corrado

Costa, David Schellenberger

Costa, Giosuè

Costa, Luca

Costa, Marina C.

Costa, Paulo Jorge

Costa, Pedro

Costa, Rosaria

Costa, Ruben

Costa, Rui

Costa, Stefania

Costa, Susana P. G.

Costabile, Gabriella

Costantini, Aniello

Costantini, Susan

Costanzo, Paola

Costas, Miguel

Coste, Franck

Costea, Teodora

Costello, Ben De Lacy

Costi, Maria Paola

Costinel, Diana

Costisor, Otilia

Cotelle, Philippe

Cotillas, Salvador

Couderc, François

Coulouarn, Cédric

Courdavault, Vincent

Couris, Stelios

Coutinho, Henrique Douglas Melo

Couto, Cristina

Covino, Roberto

Cox, James

Cox, Jonathan A. G.

Coy-Barrera, Ericsson

Coz, Alberto 


\author{
Cozza, Giorgio \\ Cozzi, Cristina \\ Cozzolino, Daniel \\ Cragg, Peter \\ Cran, Marlene J. \\ Cranfield, Charles \\ Crans, Debbie \\ Crauste, Céline \\ Cravero, Maria Carla \\ Cravotto, Giancarlo \\ Crawford, Jagoda \\ Crawford, Philip W. \\ Creager, Stephen E. \\ Creary, Xavier \\ Crescenzi, Elvira \\ Crescenzi, Orlando \\ Crespi, Stefano \\ Crespo, Irene \\ Cretoiu, Dragos \\ Creutz, Sidney \\ Crezee, Johannes \\ Criado-Gonzalez, Miryam \\ Crinelli, Rita \\ Crișan, Gianina \\ Crisan, Luminita \\ Crisp, Ryan W. \\ Crispi, Stefania \\ Cristea, Gabriela \\ Cristiano, Maria De Lurdes \\ Cristofolini, Luigi \\ Cristóvão, Beata \\ Crivii, Carmen \\ Croatt, Mitchell P. \\ Crocetti, Letizia \\ Crochet, Aurélien \\ Cross, Carroll E. \\ Crucho, Carina Isabel Correia \\ Crupi, Pasquale \\ Crupi, Rosalia \\ Cruz, Rodrigo \\ Cruz, Rui M. S. \\ Cruz-Rivera, Edwin \\ Cryle, Max \\ Csámpai, Antal \\ Csapo, Edit \\ Csávás, Magdolna \\ Csiszár, Agnes \\ Csóka, Ildikó \\ Csősz, Éva \\ Csuk, René \\ Cuadrado, Carmen
}

Cuccovia, Iolanda Midea

Cucinotta, Francis

Cuesta, Rafael

Cui, Hai-ying

Cui, Huaqing

Cui, Hui

Cui, Jingnan

Cui, Qingbin

Cui, Yuhai

Cui, Zheng

Cui, Zhisong

Cummins, Adrian

Cundari, Thomas R.

Cuny, Gregory D.

Cuomo, Francesca

Curcio, Manuela

Currò, Monica

Curti, Claudio

Curtis, Anthony D. M.

Curtis, Marion

Curulli, Antonella

Cutignano, Adele

Cutone, Antimo

Cuya, Ricardo Teobaldo

Cuzzocrea, Salvatore

Cvrček, Ladislav

Cwalina, Beata

Cyboran-Mikołajczyk, Sylwia

Cycoń, Mariusz

Cyplik, Paweł

Cypryk, Marek

Cysewski, Piotr

Cytlak, Urszula

Czarnecki, Mirosław Antoni

Czech, Bożena

Czechowski, Tomasz

Czekala, Wojciech

Czekelius, Constantin

Czekster, Clarissa M.

Czernek, Jiří

Czerwińska, Ewa

Czerwińska, Monika

Czerwiński, Marcin

Czogalla, Aleksander

Czugler, Mátyás

Czyż, Jarosław

Czyżowska, Agata

D. Bortner, Carl

Đ. Glišić, Biljana

D. Škrbić, Biljana

D'Abramo, Marco 
D'Agostino, Carmine

D'Alessandro, Walter

D'alonzo, Daniele

D'Amato, Chiara Anna

D'andrea, Cristiano

D'Angelo, Michele

D'Angelo, Stefania

D'Anna, Francesca

D'Antona, Nicola

D'Archivio, Angelo Antonio

D'Arcy, Padraig

D'Auria, John C.

D'Auria, Maria Valeria

D'Auria, Maurizio

D'Errico, Stefano

D'Onofrio, Luca

D'Onofrio, Nunzia

D'Orazio, Giovanni

D'Orsi, Beatrice

D’Ursi, Anna Maria

D'Ursi, Pasqualina

D'Urso, Alessandro

Da Costa Ribeiro, Ana Paula

Da Costa, Ana Maria Dos Santos Rosa

Da Cunha, Mário Antônio Alves

Da Pieve, Chiara

Da Silva Júnior, Arnóbio Antonio

Da Silva Júnior, Eufrânio N.

Da Silva, Emerson Rodrigo

Da Silva, Jaime Pedro Oliveira

Da Silva, Luís C. N.

Da Silva, Luis Cláudio Nascimento

Da Silva, Maria D. M. C. Ribeiro

Da Silva, Victor Diogenes Amaral

Da Silva, Wilton

Da Veiga Junior, Valdir Florencio

Da'na, Enshirah

Daberdaku, Sebastian

Dąbrowska, Monika

Dąbrowski, Wojciech

Daca, Agnieszka

Dacarro, Giacomo

Dadachova, Ekaterina

Dadáková, Kateřina

Dagmara, Malina

Dagnac, Thierry

Dahan, Albert

Dahiya, Rajiv

Dahl, Morten

Dai, Chongshan

Dai, Lizong
Dai, Yumin

Daiguebonne, Carole

Dakshanamoorthy, Arivuoli

Dal Corso, Alberto

Dal Piaz, Fabrizio

Dal Prá, Ilaria

Dalgarno, Scott

Dalhaimer, Paul

Dall'Acqua, Stefano

Dalla Pozza, Elisa

Dalla Via, Lisa

Dallavalle, Sabrina

Dalle Vacche, Sara

Dalmoro, Annalisa

Dalpozzo, Renato

Dama, Murali

Damalanka, Vishnu

Damasco, Jossana

Damberger, Fred

Dambolena, José S.

Damha, Masad J.

Damiano, Fabrizio

Damkaci, Fehmi

Damodaran, Krishna K.

Dams-Kozlowska, Hanna

Dan, Dobrota

Danac, Ramona

Danaher, Martin

Danao, Mary-Grace C.

Dance, Ian

Danciu, Corina

Dandawate, Prasad

Dandlen, Susana Anahi

Dangal, Shree

Dangles, Olivier

Dangol, Manita

Daniel, Morales Cano

Daniela, Jakšić

Danielak, Dorota

Daniel-da-Silva, Ana Luísa

Daniels, Rolf

Danielson, Neil

Danijela, Bogner

Danilo, Belviso Benny

Danilov, Sergei M.

Danjou, Pierre-Edouard

Danko, Martin

Dannaoui, Eric

Dansette, Patrick M.

Dante, Silvia

Darabantu, Mircea 
Daran, Jean-Claude

Darchen, André

Darder, Margarita

Dardonville, Christophe

Darenskaya, M. A.

Darie-Nita, Raluca Nicoleta

Daroch, Maurycy

Darras, Anastasios

Darzi, Saeedeh

Das, Pradip

Das, Rupali

Datta, Rupsa

Datta, Sandipan

Datta, Shubhashis

Daugrois, Jean-Heinrich

Davalieva, Katarina

Davank, Vadim

Davarpanah, Afshin

David, Gabriela Iulia

David, Victor

Davidescu, Corneliu-Mircea

Davies, Michael

Davinelli, Sergio

Davis, Frank

Davis, Fred

Davis, Matthew C.

Davydova, Viktoriya

Dawe, Louise

Dawn, Arnab

Dayen, Jean François

Dayneko, Sergey

Daza-Navarro, Paula

De Agostini, Ariane

De Almeida, Roberto Farina

De Almeida, Wagner Batista

De Alvarenga, Elson Santiago

De Amici, Marco

De Araújo, Daniele Ribeiro

De Araujo, Elvin

De Assis, Leonardo V. M.

De Bellis, Luigi

De Benedetto, Giuseppe

De Berardis, Domenico

De Brevern, Alexandre G.

De Bruijn, Henriette S.

De Carvalho Santos Ebinuma, Valéria

De Carvalho, Rosemary Aparecida

De Castro Ramalho, Teodorico

De Castro, Gabriela S.

De Castro, Sonia

De Ceballos, Maria L.
De Clercq, Erik

De Coninck, Joël

De Crisci, Antonio

De Deurwaerdere, Philippe

De Diego, Marta

De Falco, Bruna

De Falco, Enrica

De Falco, Valentina

De Feo, Vincenzo

De Filippis, Barbara

De Giglio, Elvira

De Giorgi, Maria Luisa

De Giorgio, Francesca

De Giudici, Giovanni

De Graaf, Coen

De Jonghe, Steven

De Julián Ortiz, Jesus

De Kruijff, Robin M.

De La Fuente, Hortensia

de la Fuente-Núnez, César

De La Garza, Mireya

De La Luz Garcia-Hernandez, Maria

De La Rosa, Laura Alejandra

De La Torre, Gema

De La Vega, José Manuel García

De Lima Nobre, Marcos Augusto

De Lima, Valéria Marçal Felix

De Luca, Giuseppina

De Luca, Pierantonio

De Luca, Stefania

De Marco, Federico

De Martin, Rainer

De Masi, Luigi

De Matteis, Valeria

De Meijer, Mari

De Melo, Marcelo M. R.

De Mendonça, Dina I. M. D.

De Mey, Gilbert

De Miranda, João Antônio Leal

De Munno, Giovanni

De Oliveira Lobo, Anderson

De Oliveira, Helinando Pequeno

De Oliveira, José Miguel P. Ferreira

De Oliveira, Kléber T.

De Oliveira, Marcos Roberto

De Oliveira, Paulo Renato

De Oliviera Silva, Eliane

De Palma, Laura

De Pasquale, Claudio

De Paula, Haroldo César Beserra

De Paz, Antonio Tabernero 
De Riccardis, Francesco

De Rosa, Igor M.

De Rosa, Maria

De Rosa, Maria Cristina

De Santo, Maria Penelope

De Silva, Franklyn

De Simone, Claudio

De Simone, Salvatore

De Simone, Salvatore Giovanni

De Sousa, Damião Pergentino

De Sousa, Frederico B.

De Souza Gil, Eric

De Souza, Danival José

De Stefano, Vita

De Tommasi, Nunziatina

De Vendittis, Emmanuele

De Villiers, Melgardt M.

De Vincentis, Giuseppe

De Visser, Samuel

De Vleeschouwer, Freija

De Vries, Mattanjah

De Wit, Maryna

De Oliveira, Ronaldo Nascimento

De, Ranjit

Deacon, Glen

Deagostino, Annamaria

Dean, Frank

Deazevedo, Eduardo

Deb, Subrata

Debnath, Asim

Dębowski, Marcin

Dec, Jan

Decurtins, Silvio

Defant, Andrea

Degennaro, Leonardo

Degiacomi, Matteo T.

Degl'innocenti, Donatella

Degola, Francesca

Dehaen, Wim

Dehelean, Cristina

Dei, Silvia

Deigner, Hans-Peter

Deja, Stanislaw

Del Caro, Alessandra

Del Castillo-Santaella, Teresa

Del Fattore, Andrea

Del Pozo Bayón, Maria A.

Del Río Celestino, Mercedes

Del Rio, Gabriel

Del Vas, Mariana

Delaglio, Frank
DelaGrecca, Marina

Delattre, Cédric

Delaude, Lionel

Delbarre-Ladrat, Christine

Delfino, Domenico V.

Delft, Floris

Delgado, Daniel Ricardo

Delgado, Juan Luis

Delgado, Sergio Nogales

Delgado-Aguilar, Marc

Delgado-Andrade, Cristina

Dell'Agli, Mario

Dell'Anna, Maria Michela

Della Ca', Nicola

Della Sala, Paolo

Dellafiora, Luca

DellaGreca, Marina

Delle Piane, Massimo

Delogu, Giovanna

DeLong, Robert K.

Delsuc, Marc-André

Dembinski, Roman

Dembska, Anna Renata

Demchuk, Oleg M.

Deme, Pragneya

Demeunynck, Martine

Demey, Hary

Demir, Fatih

Demo, Pavel

Demonacos, Constantinos

Demopoulos, Vassilis J.

Deng, Qiliang

Deng, Wen

Deng, Yongming

Denisow, Bozena

Denkov, Nikolai

Denkova, Antonia

Denny, Bill

Dent, Maja

Deo, Permal

Deo, Randhir P.

Deodato, Davide

Deora, Girdhar

Depciuch, Joanna

Derda, Ratmir

Derewiński, Mirosław

Dergunov, Sergey

Deriu, Marco Agostino

Derkatch, Svetlana

Derkowska-Zielinska, Beata

Dernovics, Mihaly 
Derosa, Joseph

Derreumaux, Philippe

Derry, Paul

Deryabina, Yu I.

Desai, Dhimant

Desgranges, Lionel

Deshpande, Shayu

Deslandes, Eric

Desmaële, Didier

Desmarets, Christophe

Dessbesell, Luana

Dessolin, Jean

Destainville, Nicolas

Detsi, Anastasia

Dettin, Monica

Dettmer, Ulf

Dettori, Maria Luisa

Deutsch, Alexander

Devasurendra, Amila

Devaurs, Didier

Devesa, Jesús

Devi, Lakshmi

Devillers, James

Devkota, Hari Prasad

Dey, Amit Kumar

Dhanasekaran, Murali

Dhasarathy, Archana

Dhasmana, Anupam

Di Bella, Santo

Di Bussolo, Valeria

Di Cerbo, Alessandro

Di Cesare Mannelli, Lorenzo

Di Chenna, Pablo

Di Giacomo, Silvia

Di Giallonardo, Francesca

Di Gioia, Maria Luisa

Di Giosia, Matteo

Di Girolamo, Rocco

Di Liegro, Italia

Di Maro, Antimo

Di Martino, Piera

Di Mattia, Carla Daniela

Di Mauro, Maria

Di Micco, Simone

Di Natale, Concetta

Di Nunzio, Francesca

Di Paola, Rosanna

Di Pasqua, Laura Giuseppina

Di Pietro, Sebastiano

Di Salle, Anna

Di Sanzo, Rosa
Di Sarli, Valeria

Di Sotto, Antonella

Di Stefano, Stefano

Di Zazzo, Erika

Di, Rong

Diaba, Faïza

Diaconeasa, Zorita

Diamantopoulos, Georgios

Diana, Patrizia

Diana, Rosita

Dias, Fábio De Souza

Dias, Maria Celeste

Dias, Maria Ines

Dias-Junior, Carlos A.

Diaz Blanco, Manuel Jesús

Diaz, Elena

Diaz, Fernando

Diaz, Sebastian Andres

Diaz-maroto Hidalgo, María Consuelo

Diaz-Marrero, Ana R.

Díaz-Ortiz, Ángel

Diban, Nazely

Dickens, Alex

Dickert, Franz L.

Dictor, Marie-Christine

Diederich, Marc

Diego, La Mendola

Díez, David

Dijkstra, Bauke

Dikalov, Sergey

Dileep, Vishnu

Diliën, Hanne

Dillon, Heather

Dima, Cristian

Dimakis, Nicholas

Dimakopoulos, Yannis

Dimandja, Jean-Marie

Dimarogona, Maria

Dimesso, Lucangelo

Dimmock, Jonathan R.

Dimos, Konstantinos

Dinache, Andra

Dinadayalane, Tandabany

Ding, Jianxun

Ding, Zhaoyang

Dingley, Andrew J.

Dini, Irene

Dini, Luciana

Dinic, Jelena

Dinică, Rodica

Dinica, Rodica-Mihaela 
Dinis, Maria Alzira Pimenta

Dinischiotu, Anca

Dinis-Oliveira, Ricardo Jorge

Dinos, Georgios

Dinu-Pirvu, Cristina-Elena

Diogo, Patricia

Diomede, Francesca

Dionisio, Giuseppe

Dionysopoulos, Dimitrios

Dippong, Thomas

Direito, Rosa

Diring, Stephane

Dissanayaka, Waruna

Divan, Ralu

DiVerdi, Joseph

Dixon, David

Dixon, David A.

Dixon, Nicholas

Djavaheri-Mergny, Mojgan

Djuran, Miloš I.

Dludla, Phiwayinkosi V.

Długaszewska, Jolanta

Dłuska, Ewa

Dmochowski, Ivan

Do Carmo, Sergio J. C.

Dobchev, Dimitar A.

Dobosz, Robert

Dobrikova, Anelia G.

Dobrovolskaia, Marina

Dobrowolski, Piotr

Dobrzynski, Piotr

Dobslaw, Daniel

Docimo, Teresa

Doda, Sai Reddy

Dodero, Veronica

Dodi, Gianina

Dogra, Pranay

Doh, Kyung-Oh

Dohanosova, Jana

Dohi, Toshifumi

Dolashka, Pavlina

Dolegowska, Barbara

Dolezal, Martin

Dolganov, Pavel Vladimirovich

Doligalska, Maria

Dołowy, Małgorzata

Dolzhenko, Anton V.

Dołżonek, Joanna

Domagała, Małgorzata

Domb, Avi

Dombrowski, Yvonne
Doménech-Carbó, Antonio

Domin, Helena

Domingues, Fernanda Da Conceição

Domingues, Sergio H.

Domingues, Valentina F.

Domínguez-Álvarez, Enrique

Domínguez-Perles, Raúl

Domínguez-Ramírez, Julio Lenin

Dominguez-Robles, Juan

Dominici, Franco

Dominikowska, Justyna

Domitrović, Robert

Donadel, Giulia

Donadu, Matthew Gavino

Donaire, Livia

Donaldson, William

Donato, Paola

Donato, Rosario

Donato, Rosario Francesco

Dong, Alideertu

Dong, Bo

Dong, Guangneng

Dong, Hai

Dong, Qiuchen

Dong, Renhao

Dong, Xinglong

Dong, Yi

Dong, Yuesheng

Donizetti, Aldo

Donnelly, Sheila

Donninger, Howard

Donnini, Sandra

Donohoe, Bryon S.

Donzelli, Elisabetta

Doody, Karen M.

Dorado, Ruben

Dormán, György

Dorotkiewicz-Jach, Agata

Dos Reis, Paulo Nobre Balbis

Dos Santos Lima, Marcos

Dos Santos, António Moreira

Dos Santos, Catarina

Dos Santos, Demetrio Jackson

Dos Santos, José Cleiton Sousa

Dos Santos, Lucilene Delazari

Dosche, Carsten

Doseff, Andrea I.

DOSOKY, NOURA

Dotsikas, Yannis

Doucet, Jean

Doudin, Khalid 
Douglas, Timothy

Douroumis, Dennis

Doušová, Barbora

Dovinova, Ima

Dowling, Jennifer

Drabik, Anna

Drabińska, Natalia

Drabowicz, Jozef

Dračínský, Martin

Dracopoulos, Vassilios

Dragos, Horvath

Dragostin, Oana Maria

Dragsted, Lars

Drasar, Pavel B.

Drescher, Simon

Dreschers, Stephan

Dressel, Martin

Drevinskas, Tomas

Drew, Michael

Drewniak, Sabina

Drgan, Viktor

Drijfhout, Falko

Drillet, Jean-François

Drochioiu, Gabi

Drolet, Marc

Drouet, Christian

Drougard, Anne

Drozd, Marek

Druzbicki, Kacper

Drużyńska, Beata

Drzeżdżon, Joanna

Du Plessis, Heinrich W.

$\mathrm{Du}$, Chengran

$\mathrm{Du}$, Deguo

$\mathrm{Du}$, Guodong

Du, Ming

Du, Zhen-Xia

$\mathrm{Du}$, Zhifeng

Duarte, Ana Rita C.

Duarte, Fátima

Duarte, Filomena L.

Duarte, Iola F.

Duarte, Maria Cristina

Duarte, Noelia

Duatti, Adriano

Dubiel, Wolfgang

Dubrova, Yuri

Duburs, Gunars

Duchet-Rumeau, Jannick

Ducho, Christian

Duda, Marcel Matei
Dudás, Zoltán

Dudek, Katarzyna

Dudev, Todor

Dudhipala, Narendar

Dudina, Dina

Dudyński, Marek

Duefer, Martina

Duggan, Brendan M.

Dulama, Ioana-Daniela

Dulf, Francisc

Duliu, Octavian

Dulova, Niina

Duma, Luminita

Dumas, Jerald

Dumbrava, Anca

Dumitriu, Raluca-Petronela

Dumitru, Gabriela

Dumont, Joseph

Dunderdale, Gary J.

Dunphy, Michael J.

Duodu, Kwaku G.

Duplay, Joëlle

Dupureur, Cynthia M.

Durán, Alexandra García

Duran, Cristhian

Durán-Peña, María Jesús

Duranti, Andrea

Durazzo, Alessandra

Durazzo, Marilena

Durgo, Ksenija

Đurović, Saša

Dusny, Christian

Dussault, Patrick

Dutartre, Patrick

Dutot, Mélody

Dutta, Aditya

Dutta, Kingshuk

Dutu, Ligia

Duval, Raphaël E.

Duval, Romain

Dux, Mária

Duzgunes, Nejat

Dvoranova, Dana

Dwamena, Amos

Dworatzek, Elke

Dwyer-Nield, Lori D.

Dydio, Paweł

Dymińska, Lucyna

Dyszkiewicz-Konwińska, Marta

Dzade, Nelson

Džeja, Petras 
Dziadas, Mariusz Tomasz

Dziegiel, Piotr

Dziekońska-Kubczak, Urszula

Dziendzikowska, Katarzyna

Dzierwa, Andrzej

Dziki, Dariusz

Dzikuć, Maciej

Dzimira, Stanisław

Dziurka, Michal

Dzolic, Zoran

Dzuba, Sergei Andreevich

Dżugan, Małgorzata

Dzyuba, Sergei

Ebani, Valentina Virginia

Ebrahim, Weaam

Ebrahimi Warkiani, Majid

Ebrahimi, Amin

Echevarria, Aurea

Echeverria, Fernando

Eckl, Katja

Eda, Shigetoshi

Eddy, Matthew T.

Ederson Rossi, Abaide

Edin, Hans

Edirisinghe, Mohan

Edreira, Martin M.

Edwards, Charles

Edwards, Christine

Edwards, Dylan R.

Edwards, Robert

Effantin, Grégory

Efremenko, Elena

Egami, Hiromichi

Egawa, Yuya

Eggleston, Ian

Egito, Eryvaldo Socrates Tabosa Do

Egorin, Andrei

Egorova, Ksenia

Ehlers, Peter

Ehm, Christian

Ehrlich, Hermann

Ehrmann, Andrea

Ehtezazi, Touraj

Eid, Nabil

Eidenberger, Thomas

Eiroa, José Luis

Eisenreich, Wolfgang

Ejidike, Ikechukwu P.

Ekaterina, Bartashevich

Ekielski, Adam

Ekino, Keisuke
Eklund, Patrik C.

El Badawy, Amro M.

El Ghoch, Marwan

El Khalloufi, Fatima

El Rawas, Rana

El-Aarag, Bishoy Y.A.

Elaissari, Abdelhamid

El-Amri, Chahrazade

Elansary, Hosam O.

El-Bacha, Tatiana

El-Dars, Farida

Eldeeb, Mohamed

Eldridge, Sandy

Eleftheriadis, Theodoros

Eleftheriou, Eleftherios P.

Elena-Herrmann, Bénédicte

Elfalleh, Walid

Eliav, Ephraim

Eliseeva, Svetlana V.

Elizalde, Luis E.

Eljaafari, Assia

Elkabets, Moshe

Eller, Fred

Ellett, Felix

Elliott, Paul I.P.

Ellison, Michael

El-Moghazy, Ahmed

Elmquist, Randolph E.

El-Nabulsi, Rami Ahmad

El-Safty, Sherif

Elsebai, Mahmoud

El-Seedi, Hesham

Elshafie, Hazem S.

Elshamy, Abdelsamed

Elsner, Matthias

Emberson, David

Emwas, Abdul-Hamid

Enache, Mădălin-Iancu

Enchev, Venelin

Encinar, Jorge Ruiz

Endo, Tatsuro

Endoh, Tamaki

Enfedaque, Alejandro

Engel, W. Dieter

Engelmann, Peter

Enko, Dietmar

Enkvist, Erki

Enman, Josefine

Enyedy, Éva Anna

Eo, Soo Hyung

Eparvier, Véronique 
Eppard, Elisabeth

Era, Benedetta

Eras, Jordi

Erb, William

Erben, Mauricio

Eremeyev, Victor

Eri, S. Srivatsan

Erickson, Jeffrey S.

Erickson, Timothy

Eriksson, Kristina

Eritja, Ramon

Erjavec, Gordana Nedic

Ernesto, Cortés-Castell

Ernesto-Casanova, Oscar

Errandonea, Daniel

Ersilia, Alexa

Esatbeyolgu, Tuba

Escalante, Jaime

Escames, Germaine

Escárcega Bobadilla, Martha Verónica

Escargueil, Alexandre

Escario, José

Escobedo, Galileo

Escola, José María

Escudé, Christophe

Escudero, Carlos

Escudero, Daniel

Escudero, Olga

Eseev, Marat

Eser, Bekir Engin

Eskin, Michael

Esmaeely Neisiany, Rasoul

Espada-Bellido, Estrella

Espartero, José L.

Espiña, Begoña

Espinach, Francesc Xavier

Espindola, Laila Salmen

Espínola, Francisco

Espinós, Carmen

Espinosa, Eduardo

Espita, Paula

Esposito, Emilio Xavier

Esposito, Germana

Esposito, Veronica

Espuelas, Socorro

Esquifino, Ana Isabel

Esquivel, Baldomero

Esquivel, Karen

Estay, Humberto

Estelrich, Joan

Esteve-Romero, Josep
Esteves, Eduardo

Estévez Cabanas, Ramón J.

Estevez, Ana

Estévez, Jorge

Estevinho, Leticia M.

Etienne, Thibaud

Etinski, Mihajlo

Eto, Bruno

Ettayapuram Ramaprasad, Azhagiya Singam

Eu, Peter

Eudald, Casals

Eugenio, Aprea

Eugster, Philippe

Evanno, Laurent

Evans, Christopher

Evans, Stephen

Evans, William

Evaristo, Jaivime

Evergren, Emma

Ewald, Andrea

Eyer, Luděk

Eyres, Graham

Ezekiel, Uthayashanker

Ezhov, Marat

Ezquerra-Brauer, Josafat-Marina

F. G. Dias, Fernanda

Fábián, István

Fabian, Zsolt

Fabiani, Roberto

Fabianowski, Wojciech

Fabio, Marmottini

Fabiszewska, Agata

Fabroni, Simona

Facchetti, Giorgio

Faccio, Greta

Fadda, Angela

Fages, Jacques

Faia, Arlete Mendes

Fairfull-Smith, Kathryn

Faissner, Simon

Fakis, Mihalis

Falcão, Soraia I.

Falciani, Chiara

Falcieri, Elisabetta

Falcone, Pasquale Massimiliano

Falconer, Ian R.

Falconer, Robert

Faleiro, Maria Leonor

Falqué López, Elena

Falsini, Sara

Fan, Deliang 
Fan, Jizhou

Fan, Liang

Fan, Qiaohui

Fan, Xuge

Fan, Zhijin

Fanali, Salvatore

Fang, Guor-Cheng

Fang, Liang

Fang, Marong

Fang, Mingxi

Fang, Qingming

Fang, Ronnie $\mathrm{H}$.

Fang, $Y i$

Fanizzi, Francesco Paolo

Fantacuzzi, Marialuigia

Fapyane, Deby

Farabegoli, Fulvia

Farantos, Stavros C.

Faraone Mennella, Maria Rosaria

Farcas, Anca Corina

Farcasanu, Ileana C.

Farci, Domenica

Fare, Silvia

Farias-Pereira, Renalison

Fariba, Fathi

Farina, Nicholas

Farinha, Carlos

Farjas, Jordi

Farkas, Attila

Farkas, Etelka

Farkaš, Pavol

Farmer, Patrick

Farris, Stefano

Farshbaf, Sepideh

Farzaneh, Vahid

Fasciana, Teresa

Fatás, Enrique

Fatima, Tahira

Fattuoni, Claudia

Fauconnier, Marie-Laure

Faustino, M. Amparo F.

Fausto, Rui

Favero, Lucilla

Favreau, Peter F.

Favre-Réguillon, Alain

Fazio, Alessia

Fazio, Barbara

Fazio, Enza

Fazzi, Daniele

Feás, Xesús

Fecka, Izabela
Feczkó, Tivadar

Federico, Stephanie

Fedin, Vladimir

Fedoreyev, Sergey

Fedosov, Sergey

Fedotov, Petr S.

Fedriani, Luis Esquivias

Feintuch, Akiva

Feis, Alessandro

Fekete, Agnes

Felczak, Krzysztof Z.

Feldblyum, Jeremy I.

Feldman, Steve

Felgueiras, Helena

Felipe-Sotelo, Monica

Feliu, Lidia

Felix, Anderson André

Félix, Luis

Félix, Luís M.

Feliz, Marta

Felpin, François-Xavier

Feng, Chaohui

Feng, Chao-Hui

Feng, Chia-Hsien

Feng, Lijuan

Feng, Sheng-Wei

Feng, Shuanglong

Feng, Wei-sheng

Feng, Xiaming

Feng, Yong-Lai

Fenning, David

Fenyvesi, Eva

Ferbinteanu, Marilena

Fercher, Christian

Ferdov, Stanislav

Feregrino-Pérez, Ana A.

Ferenc, Pajor

Feresin, Gabriela

Feresin, Rafaela G.

Ferey, Ludivine

Fermo, Paola

Fernadez-Bolanos, José G.

Fernandes, Alexandra

Fernandes, Antony

Fernandes, Carla Sofia Garcia

Fernandes, Elizabeth S.

Fernandes, Jolyn

Fernandes, José Oliveira

Fernandes, Maria Helena

Fernandes, Rosa Cristina Simoes

Fernandes, Tiago A. 
Fernández Barbero, Gerardo

Fernández Ramos, María Dolores

Fernandez, Antonio

Fernandez, Belen

Fernandez, Carlos

Fernández, Inmaculada

Fernández, Juan A.

Fernández, María Jesús

Fernandez, Maria Luz

Fernandez, Mercedes

Fernandez, Pablo Sebastian

Fernandez, Wadie Mahauad

Fernández-Arévalo, Mercedes

Fernandez-Garcia, Javier

Fernández-Gutiérrez, Mar

Fernandez-Lafuente, Roberto

Fernández-Lázaro, Fernando

Fernández-Lucas, Jesús

Fernández-Moreira, Vanesa

Fernandez-Salas, Jose A.

Fernández-Soto, Pedro

Fernando, Binosha

Feroci, Marta

Ferraboschi, Patrizia

Ferramosca, Alessandra

Ferrandez-Villena, Manuel

Ferrante, Claudio

Ferrante, Francesco

Ferrante, Maria Immacolata

Ferrantelli, Vincenzo

Ferraris, Dana

Ferraro, Vincenza

Ferreira, Ana M.

Ferreira, António

Ferreira, Guilherme

Ferreira, JOANA

Ferreira, Leonardo G.

Ferreira, Leonardo M.R.

Ferreira, Luisa Maria

Ferreira, Marcelo

Ferreira, Nelson

Ferreira, Olga

Ferreira, Paula M.

Ferreira, Ricardo J.

Ferreira, Sandra Regina Salvador

Ferreira, Susana

Ferreiro, Marta

Ferreiro-González, Marta

Ferrentino, Giovanna

Ferreon, Josephine

Ferrer, Belen
Ferrer, Emilia

Ferrer, Gerardo

Ferrer-Gallego, Raul

Ferreri, Carla

Ferri, Nicola

Ferrieres, Vincent

Ferrini, Francesco

Ferrini, Silvano

Ferro, Yvelise

Fershtat, Leonid

Festa, Carmen

Ficai, Anton

Fidalgo, Alexandra

Fidelibus, Corrado

Fideline, Tchuenbou-Magaia

Fiedler, Holger

Fiedot-Toboła, Marta

Fierascu, Irina

Fierro, José Fernando

Fierro, Vanessa

Fifere, Adrian

Figaj, Donata

Figueira, Flavio

Figueiral, Maria Helena

Figueiras, Ana

Figuereido Neto, Antonio

Figueroa Cardenas, Juan De Dios

Fijałkowski, Karol

Fik-Jaskółka, Marta A.

Filannino, Pasquale

Filarowski, Aleksander

Filella, Xavier

Filice, Simona

Filip, Adriana

Filip, Jaroslav

Filip, Lucian Dragos

Filipeanu, Catalin

Filipova, Inese

Filippi, Luca

Filippou, Panagiota S.

Filippov, Andrei

Filippov, Oleg A.

Fimognari, Carmela

Finelli, Carmine

Fini, Paola

Fiocco, Daniela

Fiore, Carlos E.

Fioretti, Bernard

Fiorica, Calogero

Fiorillo, Luca

Fiorin, Giacomo 
Fiorucci, Sebastien

Firlak, Melike

Fischer, Hartmut R.

Fischer, Henrike

Fischer, Holger

Fischer, Roland

Fischer, Wolfgang

Fischer-Fodor, Eva

Fisher, Carl

Fisher, Jonathan S.

Fisicaro, Emilia

Fisslthaler, Beate

Fiszer, Agnieszka

Fitta, Magdalena

Fitter, Jörg

Fitzpatrick, Anthony

Fiumara, Giacomo

Fizeșan, Ionel

Flamini, Guido

Flanagan-Steet, Heather

Fleet, George

Fletcher, Mary

Fleurat-Lessard, Paul

Flieger, Jolanta

Floerke, Ulrich

Florán, Benjamín

Flores Johnson, Emmanuel Alejandro

Flores, Ana Isabel

Flores-Morales, Virginia

Flórez-Fernández, Noelia

Florian, Hübner

Floris, Barbara

Florowska, Anna

Flythe, Michael

Focaroli, Stefano

Foley, Ann C.

Follink, Bart

Fologea, Daniel

Fonseca, Filomena

Font, Joaquim

Fontaine, Véronique

Fontana, Antonella

Fontana, Ariel

Fontana, Federico

Fontana, Juan

Fontana, Simona

Fontanals, Núria

Fontanay, Stéphane

Fontanini, Debora

Fonte, Pedro

Fontés, Michel
Forbey, Jennifer S.

Forero-Quintero, Linda S.

Formato, Marilena

Formisano, Pietro

Fornai, Francesco

Fornarini, Simonetta

Fornstedt, Torgny

Foroozandeh, Mohammadali

Forte, Claudia

Fortenberry, Ryan

Forti, Luca

Forzato, Cristina

Fossa, Paola

Foster, Leonard J.

Foster, Toshana Lauria

Foti, Claudia

Foubelo, Francisco

Fouillaud, Mireille

Fourmentin, Sophie

Fournier, Bertrand

Fousteris, Manolis

Fracassetti, Daniela

Fracasso, Giulio

Fracchiolla, Nicola Stefano

Fraga, Hugo

Fragai, Marco

Fraga-López, Francisco

Fragopoulou, Elizabeth

Fraguas-Sánchez, Ana Isabel

Fraile, Alberto

Franca, Eduardo F.

Franceschelli, Sara

Franceschi, Piero

Francesconi, M. Grazia

Francés-Monerris, A.

Francés-Soriano, Laura

Franchina, Flavio Antonio

Francioso, Antonio

Francisco, Ana Paula

Frančišković-Bilinski, Stanislav

Franckhauser, Sylvie

Franco, Camilo Andres

Franco, Carlos M.

Franco, Chris

Francolini, Iolanda

Frank, Craig L.

Frank, Eva

Frank, Jan

Frank, Luiza Abrahão

Frank, René

Frankowski, Marcin 
Frański, Rafał

Franssen, Maurice C. R.

Frantziskonis, George N.

Franzyk, Henrik

Frapiccini, Emanuela

Frasca, Loredana

Fratantonio, Deborah

Fratini, Filippo

Fratti, Rutilio A.

Frazier, Charles

Freakley, Simon

Frecer, Vladimir

Frédérick, Raphaël

Freitag, Marina

Freitas De Freitas, Lucas

Freitas, Vanessa Morais

Freitas, Vera L. S.

Frelon, Sandrine

Frenking, Gernot

Frense, Dieter

Frerich, Sulamith C.

Fresco-Cala, Beatriz María

Freudenthal, Bret D.

Frey, Kathleen

Freyria, Francesca Stefania

Frezza, Claudio

Frezza, Elisa

Frič, Jan

Friedrich, Holger B.

Friesen, J. Brent

Frija, Luís

Frippiat, Jean-Pol

Frisch, Benoît

Frisch, Kim

Frittitta, Lucia

Frizzo, Clarissa P.

Frkanec, Ruža

Fröde, Tânia Silvia

Froeyen, Mathy

Frogley, Mark

Fröhlich, Eleonore

Froldi, Guglielmina

Frolov, Andrej

Frolova, Liliya

Frolova, Tatiana S.

Frontera, Antonio

Frontera, Patrizia

Fruchon, Séverine

Fruit, Corinne

Fruk, Goran

Fry, Andrew
Frye, Stephen V.

$\mathrm{Fu}$, Liye

$\mathrm{Fu}$, Peng

$\mathrm{Fu}$, Tengfei

$\mathrm{Fu}$, Xiaoting

$\mathrm{Fu}, \mathrm{Xu}$

Fucikova, Anna

Fuentes-Rivas, Rosa María

Fuglsang, Anja Thoe

Fujihara, Hisako

Fujihara, Tetsuaki

Fujii, Hideaki

Fujii, Yoshiharu

Fujimori, Ko

Fujimura, Yoshinori

Fujioka-Kobayashi, Masako

Fujisawa, Kiyoshi

Fujisawa, Tomotsumi

Fujita, Ken-ichi

Fujita, Masaki

Fujita, Mikako

Fujita, Mitsugu

Fujita, Morifumi

Fujita, Wakako

Fujiwara, Akihiko

Fujiwara, Tomoya

Fukase, Koichi

Fukin, Georgy K.

Fuliaş, Adriana Violeta

Fulignati, Sara

Fuller, Amelia

Fulvio, Pasquale Fernando

Fumagalli, Laura

Fun, Lim Yee

Funar-Timofei, Simona

Funazukuri, Toshitaka

Funes-Ardoiz, Ignacio

Furdui, Bianca

Furlan, Alessandro

Furlani, Franco

Furlani, Maurizio

Furse, Samuel

Furuishi, Takayuki

Furukawa, Tatsuhiko

Furusawa, Hiroyuki

Furuta, Toshiaki

Furutani, Yutaka

Fusco, Roberta

Fuse, Shinichiro

Fuso, Francesco

Fuu, Sheu 
Fuwa, Haruiko

Fuxreiter, Monika

Gaan, Sabyasachi

Gabano, Elisabetta

Gabbar, Hossam A.

Gabbia, Daniela

Gabr, Moustafa

Gabriele, Bartolo

Gabrielli, Paolo

Gacesa, Ranko

Gaczynska, Maria

Gad, Annica K. B.

Gadipelli, Srinivas

Gadjanski, Ivana

Gadomski, Adam

Gadzała-Kopciuch, Renata

Gadžurić, Slobodan

Gagaoua, Mohammed

Gagat, Przemyslaw

Gago, Custódia

Gai, Chiara

Gaidau, Carmen

Gailer, Jürgen

Gain, Asit Kumar

Găină, Luiza Ioana

Gaisberger, Martin

Gajc-Wolska, Janina

Gajdács, Márió

Gajewicz-Skretna, Agnieszka

Gal, Adrian Florin

Gal, Emese

Gala, Rikhav P

Galabov, Angel

Galamba Correia, João Domingos

Galamboš, Michal

Galán, Miguel Ladero

Galanakis, Charis

Galanakis, Charis M.

Galanis, Alexis

Galasiti Kankanamalage, Anushka

Galasso, Giovanni

Gałązka-Czarnecka, Ilona

Galbács, Gábor

Galderisi, Umberto

Galeazzi, Roberta

Gales, Luís

Galhano, Alexandra

Galic, Ante

Galicia-García, Tomás

Galina, Gencheva

Galinetto, Pietro
Galione, Antony

Gałkowska, Dorota

Gall Troselj, Koraljka

Gallagher, John F.

Gallardo, Eugénia

Gallardo, M. Esther

Gallastegui, Gorka

Galletti, Camilla

Gallo, Monica

Galloni, Pierluca

Gallorini, Marialucia

Galperin, Michael Y.

Galstyan, Anzhela

Galus, Sabina

Galuska, Sebastian P.

Galuska, Sebastian Peter

Galvão, Adelino M.

Gálvez Buerba, Eva M.

Galvez, Jorge

Gálvez, Julio

Galvosas, Petrik

Galzitskaya, Oxana V.

Gambardella, Alessandro

Gambari, Roberto

Gamba-Sánchez, Diego

Gambino, Giorgio

Gambuti, Angelita

Gameiro, Paula

Gamelas, José

Gamella Carballo, Maria

Gamiño-Arroyo, Zeferino

Gan, Renyou

Gan, Ren-You

Gan, Samuel

Ganatsios, Vassilios

Ganazzoli, Fabio

Gancarz, Roman

Gandiglio, Marta

Gandomkar, Somayyeh

Ganesan, A.

Ganesan, Palanivel

Gang, Wei

Gangoli, Varun Shenoy

Ganguly, Avishek

Ganguly, Pritam

Ganguly, Samit

Ganji, Rakesh

Ganser, Christian

Gao, Bei

Gao, Boyan

Gao, Chengde 
Gao, Gary

Gao, Huile

Gao, Jin

Gao, Peiyuan

Gao, Yongfeng

Gao, Zhe

Garapati, Nagasree

Garbar, Christian

Garbayo, Inés

Garbers, Christoph

García Fernández, José Manuel

García Gómez, Sergio

Garcia Ispierto, Irina

Garcia, Antonio

Garcia, Coralia V.

Garcia, Jordi

Garcia, María Dolores

García, Marta Mesías

Garcia, Rosario

García-Alonso, Francisco Javier

García-Arredondo, Alejandro

Garcia-Benett, Alfonso

Garcia-Bernabé, Abel

Garcia-Bosch, Isaac

García-Conesa, María-Teresa

Garcia-Deibe, Ana

García-Estañ, Joaquín

Garcia-Fandino, Rebeca

García-Fresnadillo, David

Garcia-Gallego, Sandra

García-García, Rosa María

García-González, Víctor

García-Martínez, Jesús-María

García-Mena, Jaime

Garcia-Mina, Jose Maria

Garcia-Pardo, Javier

García-Ramos, Juan Carlos

Garcia-Reyes, Refugio B.

Garcia-Sosa, Alfonso

Garcia-Vallve, Santiago

Garcia-Verdugo, Ignacio

García-Viguera, Cristina

Gardikis, Konstantinos

Gargiulo, Valentina

Garncarek, Zbigniew

Garner, Charles

Garoli, Denis

Garrett Da Costa, Rafael

Garrido, Narciso M.

Garrido-Fernández, António

Garroni, Sebastiano
Garrosa, Manuel

Garstka, Malgorzata Anna

Garzoli, Stefania

Gasbarri, Carla

Gašić, Uroš

Gaspar, Cristina

Gaspar, Maria Manuela

Gáspári, Zoltán

Gasparini, Pierluigi

Gasset, Maria

Gatchell, Michael

Gatin, Andrey

Gatti, Antonietta

Gatti, Carlo

Gatto, Francesca

Gaudin, Karen

Gaudin, Valérie

Gaul, David

Gautam, Mukesh

Gautam, Rekha

Gautam, Uma

Gavara, Laurent

Gavenonis, Jason

Gaviña, Pablo

Gavira, José

Gavrila, Adina Ionuta

Gawel, Kinga

Gaweł-Bęben, Katarzyna

Gawlik-Dziki, Urszula

Gawronska-Kozak, Barbara

Gazdzicki, Pawel

Gaze, Mark N.

Gazerani, Parisa

Gazzano, Massimo

$\mathrm{Ge}$, Xin

Geana, Elisabeta-Irina

Gee, William

Geelen, Danny

Geerlof, Arie

Geffroy-Rodier, Claude

Gęgotek, Agnieszka

Gehring, Tito A.

Geisler-Lee, Jane

Gelbrich, Thomas

Gelbstein, Yaniv

Geletii, Yurii

Gellrich, Urs

Gemma, Sandra

Gendaszewska-Darmach, Edyta

Genders, Amanda

Geng, Huiling 
Gentile, Carla

Gentile, Davide

Gentile, Fabrizio

Gentile, Francesco

Gentili, Alessandra

Gentili, Denis

Georgakilas, Vasilios

George, Blassan

George, Graeme

George, Sonia

Georgi, Anett

Georgiades, Savvas

Georgiev, Vasil

Georgiev, Yordan

Georgiev, Yordan Nikolaev

Georgieva, Radostina

Georgopanos, Prokopios

Geraldo Yoneyama, Kelly Aparecida

Gerardino, Annamaria

Gerasimaitè, Rūta

Gerasimchuk, Nikolay

Gerbaux, Pascal

Gerber, Cobus

Gerbi, Vincenzo

Gerding, Heinrich

Gerdol, Marco

Gere, Attila

Geremia, Silvano

Gerislioglu, Burak

Gerken, Michael

Gerlitz, Gabi

Germ, Mateja

German, Nadezhda A.

Germanò, Maria Paola

Geronikaki, Athina

Gerosa, Gino

Gerothanassis, Ioannis P.

Gervasi, Teresa

Gervasoni, Jacopo

Gesslbauer, Bernd

Gestal, Monica

Geszke-Moritz, Małgorzata

Gewirtz, David

Ghaani, Masoud

Ghaani, Mohammad Reza

Ghalem, Bachir Raho

Ghanbarian, Behzad

Ghandi, Khashayar

Ghanotakis, Demetrios Demetrios

Ghantasala, Muralidhar

Ghasemi, Rasta
Ghavami, Saeid

Ghazzal, Mohamed Nawfal

Ghiasvand, Alireza

Ghica, Mihaela-Violeta

Ghidini, Michele

Ghilardi Lago, João Henrique

Ghirga, Francesca

Ghiviriga, Ion

Gholami, Fatemeh

Ghomi, Mahmoud

Ghosh, Arijit

Ghosh, Shobha

Ghosh, Soham

Ghosh, Soumita

Ghosh, Subrata

Giacomazza, Daniela

Giacomelli, Chiara

Giacomello, Pierluigi

Giamarchi, Philippe

Giampieri, Francesca

Giampietro, Letizia

Giancane, Gabriele

Gianetti, Thomas

Giannakas, Aris

Giannakourou, Maria C.

Giannopoulos, Georgios

Giannuzzi, Leda

Gianoncelli, Alessandra

Gianpiero, Cera

Giansanti, Francesco

Giárd, Jean Christophe

Giaretta, Elisa

Giaveno, Alejandra

Gibson, Christopher

Giebultowicz, Joanna

Giełdoń, Artur

Gieleciak, Rafal

Gierszewska, Magdalena

Gigliotti, Casimiro Luca

Gikas, Evagelos

Gil, Barbara

Gil, Cristiane Damas

Gil, Javier

Gilch, Peter

Giles, Corey

Giles, Wayne

Giliopoulos, Dimitrios

Gilje, John W.

Gillan, Edward G.

Gillespie, James W.

Gillich, Gilbert-Rainer 
Gillings, Nic M.

Gil-Martín, Emilio

Gil-Ramirez, Guzman

Giménez Bastida, Juan Antonio

Gimeno, Concepción

Gimpel, Matthias

Gimple, Ryan C.

Ginaldi, Lia

Gindl-Altmutter, Wolfgang

Giner-Casares, Juan J.

Gini, Giuseppina

Gioele, Pagot

Gioia, Claudio

Gioiello, Antimo

Gionfriddo, Emanuela

Giordano, Antonio

Giorgetti, Alejandro

Giorgi, Giorgio

Giorgi, Mauro

Giorgini, Elisabetta

Giosafatto, Concetta Valeria Lucia

Giovagnoli, Stefano

Giovannetti, Rita

Giovannini, Tommaso

Giovannucci, David R.

Giovarelli, Matteo

Giraldo, Liliana

Girault, Alban

Giri, Banabihari

Girolami, Flavia

Girolami, Marco

Girometta, Carolina Elena

Girotti, Albert W.

Gismondi, Angelo

Gitsov, Ivan

Giuberti, Gianluca

Giuffrè, Angelo Maria

Giuffre, Ottavia

Giuffrida, Daniele

Giuliani, Michele

Giuliano, Michela

Giunchedi, Paolo

Giuseppe, Paladini

Giuseppina, Basini

Giustiniano, Mariateresa

Gładkowski, Witold

Glasmacher, Birgit

Glass, Richard

Glasser, Wolfgang G.

Glattauer, Veronica

Glažar, Irena
Gleńsk, Michał

Glibowski, Paweł

Gligor, Felicia

Glinkowski, Wojciech

Glinton, Kevin

Glišić, Biljana

Gloaguen, Vincent

Głogowski, Robert

Glomm, Wilhelm R.

Glover, Stephen A.

Glukhov, Evgenia

Glukhova, Olga E.

Glushkov, Dmitrii O.

Głuszyńska, Agata

Gnat, Sebastian

Gniadek, Marianna

Gniewosz, Małgorzata

Göbel, Michael

Góbi, Sándor

Gobin, Ivana

Goclon, Jakub

Godin, Biana

Godinho, Maria Helena

Godlewska-Żyłkiewicz, Beata

Godyń, Justyna

Goff, Gaëlle Le

Goh, Guodong

Gohain, Priyakshee Borpatra

Gohda, Eiichi

Goi, Anna

Gojo, Satoshi

Golba, Sylwia

Goldeman, Waldemar

Golewski, Przemysław

Golic-Grdadolnik, Simona

Golovko, Mikhail

Goltsov, Alexey

Goltz, Douglas M.

Golub, Eyal

Golub, Igor

Gomes Da Cruz, Adriano

Gomes, Ana T. P. C.

Gomes, Carlos Martins

Gomes, Clara S. B.

Gomes, Joao F

Gomes, Marcelo Gomes De

Gomes, Nelson

Gomes, Paula A. C.

Gomes-da-Silva, Lígia C.

Gómez Barrios, Xiomar

Gómez Castellà, Cristina 
Gómez De Cedrón, Marta

Gómez García, Carlos J.

Gómez Ocampo, Iván

Gómez, Ana María

Gómez, Jose Lorenzo

Gomez, Nidia

Gómez-Ariza, José Luis

Gómez-Bengoa, Enrique

Gómez-Cámer, Juan Luis

Gómez-Cápiro, Oscar

Gomez-Pilar, Javier

Gomez-Ruiz, Santiago

Gomez-Sainero, Luisa Maria

Gomha, Sobhi

Goncalves, Alexandre A.S.

Gonçalves, Ana M. M.

Gonçalves, Bruno

Gonçalves, M. Sameiro T.

Gonçalves, Virginia

Goncharuk, Olena

Gonda, Sandor

Gondova, Tatana

Gonec, Tomas

Goněc, Tomáš

Gong, Max

Gong, Xingchu

Goni, Felix

Gonzales, Cara B.

González Domínguez, Raúl

González Laredo, Rubén F.

Gonzalez Viejo, Claudia

González, Concepción C.

González, Florenci V.

Gonzalez, Juan M.

González, M. Carmen

González, María De Las Nieves

González, Marta

Gonzalez, Oskar

Gonzalez, Victor

Gonzalez, Zoilo

Gonzalez, Zoraida

González-De-Peredo, Ana V.

González-García, Jorge

González-Laredo, Rubén Francisco

Gonzalez-Lezcano, Roberto Alonso

González-Marrero, Joaquin

González-Maya, Leticia

González-Muñiz, Rosario

González-Pérez, José A.

González-Sálamo, Javier

Gonzalez-Stuart, Armando Enrique
González-Vallinas, Margarita

González-Vergara, Enrique

Gonzalez-Vicente, Agustin

González-Zamora, Eduardo

Goormaghtigh, Erik

Gopalakrishnan, Gopakumar

Gopalakrishnan, Ranga

Góra, Artur

Gorbacheva, Liubov

Gorczynski, Adam

Goretti, Enzo

Gorgan, Lucian

Gorgieva, Selestina

Gori, Antonella

Gorinstein, Shela

Gorkunov, Maxim V.

Gornas, Pawel

Gornitzka, Heinz

Gornostaev, Leonid M.

Gornowicz, Agnieszka

Gornowicz-Porowska, Justyna

Goroncy, Alexander

Gorpas, Dimitris

Gorski, Łukasz

Gorynski, Krzysztof

Gościańska, Joanna

Goseki, Raita

Gosetti, Fabio

Gośliński, Tomasz

Gospodarek, Janina

Gossage, R. A.

Goto, Masuo

Goto, Motonobu

Goto-Inoue, Naoko

Gotor, Alicia Ayerdi

Gotor-Fernández, Vicente

Gotsbacher, Michael

Gottwald, Eric

Goua, Marie

Goudoulas, Thomas

Goula, Maria A.

Goulas, Vlasios

Gourlay, Louise J.

Gouveia, Rubia Figueredo

Gouws, Chrisna

Govan, Joseph

Gowda, Krishne

Goyanes, Alvaro

Gozin, Michael

Grabarczyk, Małgorzata

Grabarek, Beniamin Oskar 
Grabchev, Ivo

Grabek-Lejko, Dorota

Grabner, Daniel S.

Grabowski, Slawomir

Gracin, Davor

Graczyk-Jarzynka, Agnieszka

Gradoni, Luigi

Gradov, Oleg V.

Gradzielski, Michael

Graebin, Cedric

Graham, David

Gramignoli, Roberto

Gramza-Michałowska, Anna

Granados-Principal, Sergio M.

Granato, Daniel

Granchi, Carlotta

Grande Tovar, Carlos David

Grandemange, Stéphanie

Grandjean, Cyrille

Granica, Sebastian

Granja, Tiago

Grant, George

Grases, Felix

Grässel, Susanne

Grassi, Silvia

Grasso, Giuseppe

Grasso, Simona

Grattoni, Alessandro

Grause, Guido

Gray, Christophert

Graziano, Guella

Graziano, Sossio Fabio

Greatrex, Ben

Grebenc, Tine

Greco, Todd

Green, Ivan

Green, Michael

Greenberg, Robert M.

Greenhalgh, David

Greenlief, C. Michael

Greenspan, Phillip

Greenwood, Michael T.

Greer, Edyta

Greetham, Darren

Gregor, Ingo

Gregori, Alberto

Grembecka, Małgorzata

Gressent, Frédéric

Gribble, Gordon

Gribskov, Michael

Grice, Jeffrey E.
Grider, Arthur

Griesbeck, Axel G.

Griffini, Gianmarco

Griffiths, William

Grigoraš, Anca Giorgiana

Grilc, Miha

Grill, Magdalena

Grillo, Federico

Grillo, Giorgio

Grimaldi, Annalisa

Grimaldi, Giovanna

Grimm, Marcus O. W.

Grishin, Maxim

Grishko, Victoria

Grisoni, Francesca

Gritsan, Nina P.

Grivas, Ioannis

Grob, Koni

Grochowski, Cezary

Gröger, Thomas

Gronwald, Wofram

Gross, Andrew J.

Groß, Harald

Gross, Joshua B.

Grossi, Giancarlo

Grosso, Clara

Grosso, Giuseppe

Grotli, Morten

Groundwater, Paul

Grubeša, Ivanka Netinger

Grudnik, Przemyslaw

Grul'ová, Daniela

Grumet, Rebecca

Gruszczynska-Biegala, Joanna

Gruzdev, Matvey

Gryaznevich, Mikhail P.

Grygier, Anna

Gryn'ova, Ganna

Grynkiewicz, Grzegorz

Gryszkin, Artur

Grzegorczyk, Izabela

Grzyb, Joanna

$\mathrm{Gu}$, Zhen

Guadagni, Fiorella

Gualerzi, Alice

Guamis, Buenaventura

Guangjiu, Zhao

Guazzelli, Lorenzo

Gubatan, John

Gubitosa, Jennifer

Guda, Alexander A. 
Gudiminchi, Rama

Gudiña, Eduardo

Gudiukaité, Renata

Gudkov, Sergey V

Gudmundsdottir, Anna D.

Gudmundsson, Jon Tomas

Gudoshnikov, Sergey

Guedes, Rita C.

Guedes-Alonso, Rayco

Guerre, Philippe

Guerrero, José Alejandro Heredia

Guerrieri, Nicoletta

Guerrini, Alessandra

Guerrini, Gabriella

Guerrini, Marco

Guescini, Michele

Guglielmi, Paolo

Guglielmotti, Manfredi

Guha, Prasun

Guhathakurta, Subhrangshu

Gui, Rijun

Guibal, Eric

Guichard, Elisabeth

Guidi, Patrizia

Guidotti, Matteo

Guijun, Xian

Guillena, Gabriela

Guillén-Bejarano, Rafael

Guillén-Sánchez, Dominico A.

Guillet-Nicolas, Remy

Guillou, Claude G.

Guiné, Raquel

Guiot, Caterina

Guitton, Jérôme

Güldiken, Nurdan

Gulea, Mihaela

Gulic, Tamara

Gulicovski, Jelena

Gulino, Antonino

Gullo, Maurizio

Gullon, Beatriz

Gullon, Betatriz

Gulyaeva, Lyudmila

Gumieniczek, Anna

Gumienna, Małgorzata

Gumul, Dorota

Gun'ko, Vladimir M

Gun'ko, Vladimir M.

Gunaje, Jayarama

Gunasekara, Chamila

Guncheva, Maya
Gunda, Venugopal

Gundamaraju, Rohit

Gunia-Krzyżak, Agnieszka

Guńka, Piotr

Günther, Stefan

Günther, Ulrich

Guo, Chih-Hung

Guo, Dandan

Guo, Fenghai

Guo, Hao-Bo

Guo, Jingshu

Guo, Ju-Tao

Guo, Ming-quan

Guo, $\mathrm{Na}$

Guo, Ting

Guo, Weimin

Guo, Xiaoyu

Guo, Xinbo

Guo, Xingzhong

Guo, Yong

Guo, Zhan-Hu

Guo, Zhefeng

Guo, Zheng

Guo, Zong Sheng

Guochen, Bao

Gupta, Kajal

Gupta, Sanjay

Gupta, Sushim

Gurevich, Vsevolod V.

Gurikov, Pavel

Gurina, Darya

Gurrapu, Sreeharsha

Gus'kov, Vladimir

Gusarov, Sergey I.

Guselnikova, Olga Andreevna

Gushchin, Artem L.

Gushina, Irina

Gusiatin, Zygmunt Mariusz

Guskos, Niko

Guskova, Olga

Gust, Ronald

Gustafson, Kirk

Gutberlet, Thomas

Guterman, Ryan

Gutierrez Ramirez, Manuel

Gutiérrez Ramírez, Manuel

Gutiérrez, Laura

Gutierrez, Leopoldo

Gutiérrez, Margarita

Gutierrez, Osvaldo

Gutierrez-Aguilar, Manuel 
Gutiérrez-Arzaluz, Mirella

Gutiérrez-Gallego, Ricardo

Gutiérrez-Gamboa, Gastón

Gutierrez-Merino, Carlos

Gutiérrez-Venegas, Gloria

Guzik, Maciej

Guzman-Puyol, Susana

Gwalani, Bharat

Gwiazdowska, Daniela

Gyarmati, Benjámin

Gyémánt, Gyöngyi

Gyepes, Róbert

Gyöngyösi, Mariann

H. Gonzalez, Daniel

H. Moreno, Paulo Roberto

HA, Hyung-Ho

Ha, Jung-Heun

$\mathrm{Ha}$, Kwon-Soo

Habala, Ladislav

Habas, Khaled

Habata, Yoichi

Habaue, Shigeki

Habermehl-Cwirzen, Karin

Habuda-Stanić, Mirna

Hackl, Thomas

Hada, Masahiko

Hadden, Kyle

Hadian, Kamyar

Hadjikakou, Sotiris

Hadjipavlou-Litina, Dimitra

Ha-Duong, Nguyet-Thanh

Hadwiger, Jeffrey A.

Haendler, Bernard

Haertle, Thomas

Hafiane, Anouar

Hagar, Mohamed

Hagberg, Carolina

Hagelueken, Gregor

Hageman, Jos

Hahn, Friedrich

Haidar, Samer

Haidar, Ziyad S.

Haider, Norbert

Hajizadeh, Solmaz

Halász, Katalin

Halcovitch, Nathan R

Halder, Amit Kumar

Halder, Avik

Halet, Jean-François

Haliński, Łukasz P.

Hallam, Toby
Hallmann, Ewelina

Ham, Young Wan

Hamano, Yoshimitsu

Hamasaki, Yoshifumi

Hamashima, Yoshitaka

Hamba, Hidenori

Hamdan, Nasser

Hameed, Ahsan

Hamilton, Chris

Hamman, Joias

Hammarstrom, Jane

Hammer, Martin

Hammerl, Jens Andre

Hamon, Jean-René

Hampp, Norbert

Hampp, Norbert A.

Han, Bingnan

Han, Dong

Han, DongWoon

Han, Felicity

Han, Heyou

Han, Jaehong

Han, Jianlin

Han, Jinsong

Han, Jong Won

Han, Qian

Han, Quan-Bin

Han, Se Jong

Han, Soo Deok

Han, Sung Gu

Han, Xiuguo

Han, You

Han, Zhenlin

Hanai, Toshihiko

Hanania, Michel

Hanaoka, Toshiaki

Hanawa, Takehisa

Hancu, Gabriel

Handa, Akihiro

Handa, Makoto

Handzlik, Jadwiga

Hanefeld, Ulf

Haneke, Eckart

Hanemann, Thomas

Hang, Taijun

Hanganu, Daniela

Hanifehpour, Younes

Hannedouche, Jerome

Hano, Christophe

Hansen, Espen

Hansen, Niels 
Hansford, Karl

Hanton, Lyall

Hao, Jinsong

Hao, Zhao

Haouas, Mohamed

Haponiuk, Józef

Haque, Ariful

Hara, Kenji

Haraldsen, Jason T.

Harashima, Nanae

Harasym, Joanna

Harbertson, James

Hardacre, Allan

Harijan, Rajesh K

Harijith, Anantha

Harms, Amy

Harnisz, Monika

Haroun, Ricardo

Harp, Joel M.

Harper, James

Harrington, Peter De Boves

Harris, Chris

Harris, Edward N.

Harris, Trevor

Harrison, Joe

Harrison, Patrick

Harrison, Paul H. M.

Harrison, Sabine

Harrison-Bernard, Lisa

Harscoat-Schiavo, Christelle

Hart, Peter C.

Hartenbach, Ingo

Hartl, Frantisek

Hartman, Mariusz

Hartman, Matthew C. T.

Hartung, Jens

Haruki, Mitsuru

Harvey, Peta

Has, Cristina

Hasan, Abshar

Hasan, Mehedi

Hasan, Murtaza

Hasan, Syed Saif

Hasanuzzaman, Mirza

Hasegawa, Makoto

Hasegawa, Morifumi

Hashem, Abul

Hashemi, Mohtadin

Hashidzume, Akihito

Hashimoto, Makoto

Hashimoto, Masanori
Hashimoto, Masaru

Hashimoto, Takeshi

Hashimoto, Tomoko

Hashimoto, Yoshitaka

Hasiuk, Franciszek J.

Haskó, György

Hassan, Ahmed

Hassan, Errol

Hassan, Mohamed

Hassan, Sherif T. S.

Hasumi, Keiji

Hatada, Ruriko

Hatae, Noriyuki

Hatano, Akihiko

Hatano, Tsutomu

Hatano, Yuichiro

Hathaway, Nathaniel

Hati, Santanu

Hatzakis, Emmanuel

Hatzakis, Nikos

Hatzdimitriou, Effie

Hatzilazarou, Stefanos

Hatzpolous, Polydefkis

Haudecoeur, Romain

Haufe, Günter

Haugen, Håvard J.

Haupt, Michael

Haurie, Laia

Hauser, Peter

Hausmann, Michael

Havelek, Radim

Havlik, Jaroslav

Havran, Ludek

Hawash, Zafer

Hawes, Chris S.

Hawryl, Anna M.

Hawrył, Mirosław

Hay, Sam

Hayashi, Mariko Kato

Hayashi, Noriko

Hayashi, Yoshihito

Hayes, Maria

Hayes, Sidney J.

Haynes, Richard

Hayward, Steven

Hazai, László

He, Chengwei

He, Dongfeng

$\mathrm{He}$, Guanjie

He, Hailun

$\mathrm{He}$, Huijun 
He, Lixia

He, Puming

He, Wangxiao

$\mathrm{He}$, Xin

He, Yong

He, Zhiguo

Heavey, Susan

Heber, Dieter

Heberden, Christine

Hebrant, Marc

Heck, Jürgen

Hedeland, Mikael

Hedhammar, My

Hedstrom, Lizbeth

Hee, Ay Ching

Heere, Michael

Heffeter, Petra

Hegarty, Matthew

Hegde, Vijay

Hegedűs, László

Hegedűsová, Alžbeta

Heinrich, Benoît

Hejna, Aleksander

Helesbeux, Jean-Jacques

Hellweg, Thomas

Hellwig, Michael

Heltai, Gyorgy

Hémadi, Miryana

Hendriks, Hans H.

Henen, Morkos A.

Heng, Nicholas C. K.

Hengesbach, Martin

Henke, Andreas

Hennig, Sven

Henrich, Curtis J.

Henrique, Coutinho

Henriques, Bárbara J.

Henriques, Marta H. F.

Hensley, Alyssa

Heras, Francisco

Herbert, John

Herchel, Radovan

Herckes, Pierre

Herczegh, Pál

Herdewijn, Piet

Hermann, Gerrit

Hermann, Petr

Hermanowicz, Justyna

Hermenean, Anca

Hernadi, Klara

Hernández Saz, Jesús
Hernandez, Agustin

Hernández, Eliud Avier

Hernández, Francisca

Hernandez, Jesus

Hernandez, Reinier

Hernández-Barajas, José

Hernández-Carlos, Beatriz

Hernandez-Escobedo, Quetzalcoatl

Hernández-Hernández, Oswaldo

Hernández-López, Hiram

Hernández-Mesa, Maykel

Hernández-Montelongo, Jacobo

Hernández-Núñez, Emanuel

Herold, Nikolas

Herráez-Hernández, Rosa

Herranz, Fernando

Herranz, Maria Angeles

Herrebout, Wouter

Herrera Zaldivar, Manuel

Herrera, Raquel

Herrera-Herrera, Antonio V.

Herrero-Martínez, José Manuel

Herres-Pawlis, Sonja

Hertz, Daniel L.

Hetényi, Csaba

Heuer-Jungemann, Amelie

Heusch, Gerd

Hibbitts, Alan

Hibi, Takao

Hickey, Neal

Hidalgo Herrador, José Miguel

Hiejima, Yusuke

Hierro, Isabel Del

Higashi, Kyohei

Higashi, Taishi

Higashino, Tomohiro

Higgs, David

HIjikata, Atsushi

Hikawa, Hidemasa

Hikichi, Shiro

Hildreth, Eason

Hildt, Eberhard

Hill, Grant

Hill, Jonathan

Hill, Stephen

Hillard, Elizabeth

Hinds, Terry D.

Hines, Rochelle M.

Hingorani, Dina

Hinman, Samuel

Hintzen, Hubertus 
Hirahara, Masanari

Hirano, Koji

Hirano, Masafumi

Hirashita, Tsunehisa

Hirata, Tetsuya

Hirata, Yoko

Hirata-Tsuchiya, Shizu

Hiroaki, Hidekazu

Hirota, Kiichi

Hirsch, Thomas

Hirva, Pipsa

Hiscock, Jennifer

Hix, Gary

Hlaváč, Viktor

Ho, Chi-Tang

Ho, Junming

Ho, Mengfei

Ho, Wing Shing

Hocek, Michal

Hochlaf, Majdi

Hockova, Dana

Hodac, Ladislav

Hodges, H. Courtney

Hodgkinson, James

Hodgson, David R. W.

Hofbauer, Stefan

Hofer, Anders

Hoff, Bård Helge

Hoffman, Rhonda

Hofmann, Ute

Hogwood, John

Hojo, Fuhito

Holak, Tad A.

Holb, Imre

Holder, Alvin

Holec, Josef

Holen, Elisabeth

Holien, Jessica

Hollenstein, Marcel

Hollmann, Axel

Hollmann, Eric

Holmberg, Krister

Holmes, Neil

Holsinger, Damain

Holt, Roberta

Holt, Stephen A.

Holtmann, Dirk

Holynska, Malgorzata

Homaeigohar, Shahin

Homem-de-Mello, Mauricio

Homonnay, Zoltan
Honcharenko, Malgorzata

Honcová, Pavla

Hondal, Robert

Honermeier, Bernd

Hong, Ic-Pyo

Hong, Jongin

Hong, Jun

Hong, Kui

Hong, Mee Young

Hong, Yuning

Honório, Káthia

Hooda, Jagmohan

Hook, Ingrid

Hopkins, Benjamin D.

Hopkinson, Richard

Horacek, Micha

Horecka, Beata

Horhat, Florin George

Hori, Akiko

Horino, Yoshikazu

Hornak, Jaroslav

Hornak, Joseph P.

Hornemann, Thorsten

Hörner, Gerald

Horrocks, Richard

Horti, Andrew

Hortobagyi, Tibor

Horvath, Anelia

Horvath, Balazs

Horvath, Dragos

Horváth, Judit

Horzmann, Katharine

Hosein, Ian Dean

Hošek, Jan

Hoshino, Norihisa

Hosmane, Narayan

Hosokawa, Yoichiro

Hosomi, Ryota

Hosório, Hugo

Hossain, A. S. M. Monjur Al

Hossain, Ekram

Hossain, Maruf

Hossain, Md Ashraf

Hossain, Mohammad Zakir

Hoštálková, Anna

Hosur, Vishnu

Hosztafi, Sandor

Hou, Bo

Hou, Duen-Ren

Hou, Qidong

Hou, Xianghui 
Hou, Zhaosheng

Houen, Gunnar

Houjou, Hirohiko

Houng, Jer-Yiing

Houry, Walid A.

Housecroft, Catherine

Houweling, Peter

Houzé, Pascal

Howell, Bob

Howells, Lynne

Howes, Philip

Hoyos, Pilar

Hrabal, Richard

Hrckova, Gabriela

Hrenar, Tomica

Hroboňová, Katarína

Hsia, Shih-Min

Hsieh, Chang-Wei

Hsieh, Kuangwen

Hsieh, Lu-Sheng

Hsieh, Ming-Ju

Hsieh, Tusty-Juan

Hsieh, Yi-Hsien

Hsieh, Yves

Hsin, Kun-Yi

Hsu, Chih-Chieh

Hsu, Chin-Lin

Hsu, Day-Shin

Hsu, Hao-Jen

Hsu, I-Jui

Hsu, Ming-Hua

Hsu, Todd

Hsu, Yu-Kuei

Hsueh, Yi-Huang

$\mathrm{Hu}$, Changling

$\mathrm{Hu}$, Chenlin

$\mathrm{Hu}$, Fangzhong

$\mathrm{Hu}$, Fuliang

$\mathrm{Hu}$, Gaizun

$\mathrm{Hu}$, Guang

$\mathrm{Hu}, \mathrm{Guoku}$

$\mathrm{Hu}$, Guoli

$\mathrm{Hu}$, Hao

$\mathrm{Hu}$, Jiafen

$\mathrm{Hu}$, Jinming

$\mathrm{Hu}$, Min

$\mathrm{Hu}$, Qinhong

$\mathrm{Hu}$, Shang-Hsiu

$\mathrm{Hu}$, Wanhe

$\mathrm{Hu}$, Wei-Ping

$\mathrm{Hu}$, Xiang-Ping
$\mathrm{Hu}$, Xiaoqiang

$\mathrm{Hu}, \mathrm{Xiaoyu}$

$\mathrm{Hu}$, Yanmei

Huang, Anpeng

Huang, Cheng-Liang

Huang, Chia-Hua

Huang, Chi-Chang

Huang, Chih-Chia

Huang, Chih-Feng

Huang, Faqing

Huang, Genin Gary

Huang, Haw-Ming

Huang, Hui-Chi

Huang, HungJi

Huang, Jiann-Jyh

Huang, Jui-Hsien

Huang, Meng-Yuan

Huang, Peng

Huang, Rena

Huang, Riming

Huang, Timothy

Huang, Tse-Hung

Huang, Weimin

Huang, Weixin

Huang, Wen-Chung

Huang, Xiaodan

Huang, Xuefeng

Huáng, Xuri

Huang, Ya-ling

Huang, Yeou-Lih

Huang, Yifeng

Huang, Yu-Chun

Huang, Yu-Fang

Hubbe, Martin

Hubbe, Martin A.

Huber, Gaspard

Huber, Roland G.

Hubicka, Urszula

Hübner, Florian

Huc, Vincent

Huczyński, Adam

Hudalla, Gregory A.

Hudson, Robert

Hudson, Samuel

Huebschle, Christian Bertram

Huefner, Antje

Huerta, Miguel

Huertas, Jesús R.

Huh, Joo Young

Huh, Seong

Huisman, Marc 
Hułas-Stasiak, Monika

Humar, Miha

Hung, Andrew

Hung, Hsin-Yi

Hung, Shih-Ya

Hungerford, James

Hungerford, Natasha

Hunter, Luke

Hunter, Robert P.

Huntosova, Veronika

Huq, Md. Amdadul

Hurren, Christopher

Hurtado Oliva, Miguel Ángel

Husnjak, Koraljka

Hussain, Hidayat

Hussein, Ahmed

Hussein, Waleed

Husson, Jerome

Hvattum Divon, Hege

Hwang, Bang Yeon

Hwang, Eun Sook

Hwang, In Koo

Hwang, Jiun-Ren

Hwang, Kwon Tack

Hwang, Long-Chih

Hwangbo, Cheol

$\mathrm{Hwu}$, Jih Ru

Hyeyoung, Seo

Hyun, Sang-Hwan

Iacumin, Paola

Iadarola, Paolo

Iammarino, Marco

Ianchis, Raluca

Iatrou, Hermis

Iatsunskyi, Igor

Ibáñez Escribano, Alexandra

Ibáñez, Pablo Rafael Pardo

Ibrahim, Mohamed Ali

Ibrahim, Salam

Ichiishi, Naoko

Ichikawa, Satoshi

Idowu, Temilolu

Ielciu, Irina

Ientile, Riccardo

Igea, Ana

Iglesias, Alberto Á.

Iglesias, Emilia

Ignat, Maria

Ignatov, Alexander

Igne, Benoît

Ignés-Mullol, Jordi
Ignjatovic, Nenad

Iizuka, Katsumi

Ikechukwu, Ejidike

Ikeda, Masato

Ikeda, Masayuki

Ikegami, Takahisa

Ikonomopoulou, Maria

Ikura, Teikichi

Ikuta, Naoko

Il'yushin, Mikhail A.

Ilaš, Janez

Ilia, Gheorghe

Ilic, Nebojsa

Ilic-Stojanovic, Snezana

Ilinskiy, Yuriy

Ilisz, Istvan

Ilisz, István

Illa, Ona

Illescas, Javier

Im, Hyungsoon

Imai, Hiroo

Imai, Jun

Imam, Syed

Imamoto, Yasushi

Imamura, Masahiro

Imberti, Cinzia

Imhof, Petra

Imlach, Wendy L.

Imm, Jee-Young

Imoto, Kenta

Imperatore, Concetta

Improta, Roberto

Imre, Silvia

Inaba, Hiroshi

Inagaki, Hidetoshi

Inchingolo, Francesco

Ingallina, Cinzia

Ingelman-Sundberg, Magnus

Inglese, Paolo

Ingram, Conrad

Inguimbert, Nicolas

Inokuchi, Tsutomu

Inokuma, Tsubasa

Inomata, Hiroshi

Inomata, Katsuhiro

Inoue, Atsuko

Inoue, Hirofumi

Inoue, Keiichi

Inui, Masayuki

Inuzuka, Toshiyasu

Inyushin, Mikhail Y. 


\begin{tabular}{|c|c|}
\hline Ioannides, Theophilos & Ivančić Šantek, Mirela \\
\hline Ion, Rodica-Mariana & Ivanov, Sergey \\
\hline Ionescu, Elena Rodica & Ivanov, Yuri D. \\
\hline Ionescu, Mihaela Ileana & Ivanova, Alla V. \\
\hline Ionete, Roxana Elena & Ivanova, Donika G. \\
\hline Ionita, Petre & Ivanova, Svetlana \\
\hline Ionut, Ioana A. & Ivashchenko, Olena \\
\hline Iordache, Florin & Iwai, Atsushi \\
\hline Iorizzi, Maria & Iwaoka, Michio \\
\hline Iorizzo, Massimo & Iwata, Yukiyoshi \\
\hline Ippoliti, Thomas & Izaguirre, Gonzalo \\
\hline Iqbal, Hafiz M. N. & Izumi, Kouji \\
\hline Iram, Surtaj & Izumi, Yoshihiro \\
\hline Irazoqui, Javier & Izzo, Irene \\
\hline Iriepa Canalda, Isabel & J Edagwa, Benson \\
\hline Irina, Carlescu & Jabin, Ivan \\
\hline Iriondo, Amaia & Jablan, Jasna \\
\hline Irwin, David C. & Jablonska, Karolina \\
\hline Isakova-Sivak, Irina & Jabłońska-Trypuć, Agata \\
\hline Isaza Martínez, José Hipólito & Jablonski, Miroslaw \\
\hline Iseppi, Ramona & Jabraoui, Hicham \\
\hline Ishihara, Kazuaki & Jacak, Jaroslaw \\
\hline Ishihara, Osamu & Jacevic, Vesna \\
\hline Ishii, Daisuke & Jacinto, Estela \\
\hline Ishii, Takahiro & Jackai, Louis E. N. \\
\hline Ishikawa, Ryuta & Jackowski, Karol \\
\hline Ishimizu, Takeshi & Jackson, Robert A. \\
\hline Ishitsuka, Yoichi & Jackson, William F. \\
\hline Ishizuka, Takumi & Jacob, Christophe \\
\hline Islam, Md Soriful & Jacob, Gopas \\
\hline Islam, Zohorul & Jacob, Paul \\
\hline Islas, Leon & Jacobo-Velazquez, Daniel \\
\hline Islas-Jácome, Alejandro & Jacobsen, Elisabeth \\
\hline Ismael, Maria Isabel & Jacomin, Anne-Claire \\
\hline Isman, Murray B. & Jacquemin, Denis \\
\hline Isoda, Kyosuke & Jadeja, Ravirajsinh \\
\hline Isono, Takuya & Jaffrezic-Renault, Nicole \\
\hline Isvoran, Adriana & Jaganjac, Morana \\
\hline Itakura, Yoko & Jager, Martine J \\
\hline Itatani, Yoshiro & Jagerovic, Nadine \\
\hline Iticescu, Catalina & Jagminas, Arunas \\
\hline Ito, Michiho & Jain, Ankit \\
\hline Ito, Naoki & Jain, Gaurav \\
\hline Ito, Shosuke & Jaiswal, Jagdish Kumar \\
\hline Ito, Takuya & Jaja, Ishmael Festus \\
\hline Ito, Yoichiro & Jakas, Andreja \\
\hline Itoh, Toshiyuki & Jaki, Birgit \\
\hline Itoh, Yoshifumi & Jakić, Miće \\
\hline Itsuno, Shinichi & Jakimovski, Dejan \\
\hline Iturriaga-Vasquez, Patricio & Jakobek, Lidija \\
\hline Iván, Béla & Jakobusic Brala, Cvijeta \\
\hline
\end{tabular}

Ioannides, Theophilos

Ion, Rodica-Mariana

Ionescu, Elena Rodica

Ionescu, Mihaela Ileana

Ionete, Roxana Elena

Ionita, Petre

Ionut, Ioana A.

Iordache, Florin

orizzi, Maria

Ippoliti, Thomas

Iqbal, Hafiz M. N.

Irazoqui, Javier

Iriepa Canalda, Isabel

Irina, Carlescu

Iriondo, Amaia

Irwin, David C.

Isakova-Sivak, Irina

Isaza Martínez, José Hipólito

Iseppi, Ramona

Ishihara, Kazuaki

Ishihara, Osamu

Ishii, Daisuke

Ishii, Takahiro

Ishikawa, Ryuta

Ishimizu, Takeshi

Ishitsuka, Yoichi

Ishizuka, Takumi

Islam, Md Sorifu

Islas-Jácome, Alejandro

Ismael, Maria Isabe

Isman, Murray B.

Isoda, Kyosuke

Isono, Takuya

Isvoran, Adriana

Itakura, Yoko

Itatani, Yoshiro

Iticescu, Catalina

Ito, Michiho

Ito, Naoki

Ito, Shosuke

Ito, Takuya

Itoh, Yoshifumi

Itsuno, Shinich

Iván, Béla
Ivančić Šantek, Mirela

Ivanov, Sergey

Ivanov, Yuri D.

Ivanova, Alla V.

Ivanova, Donika G.

Ivanova, Svetlana

Ivashchenko, Olena

Iwai, Atsushi

Iwaoka, Michio

Iwata, Yukiyoshi

Izumi, Kouji

mi, Yoshihiro

Jabin, Ivan

Jablan, Jasna

Jablonska, Karolina

Jabłońska-Trypuć, Agata

Jablonski, Miroslaw

Jabraoui, Hicham

Jacak, Jaroslaw

Jacevic, Vesna

Jacinto, Estela

Jackai, Louis E. N.

Jackowski, Karol

Jackson, Robert A.

Jackson, William F.

Jacob, Gopas

, Pau

Jacobsen, Elisabeth

Jacomin, Anne-Claire

Jacquemin, Denis

Jadeja, Ravirajsinh

Jaffrezic-Renault, Nicole

Jaganjac, Morana

Jager, Martine J

Jagerovic, Nadine

Jagminas, Arunas

Jain, Ankit

Jain, Gaurav

Jaiswal, Jagdish Kumar

Jaja, Ishmael Festus

Jakas, Andreja

Jaki, Birgit

Jakić, Miće

Jakobek, Lidija

Jakobusic Brala, Cvijeta 


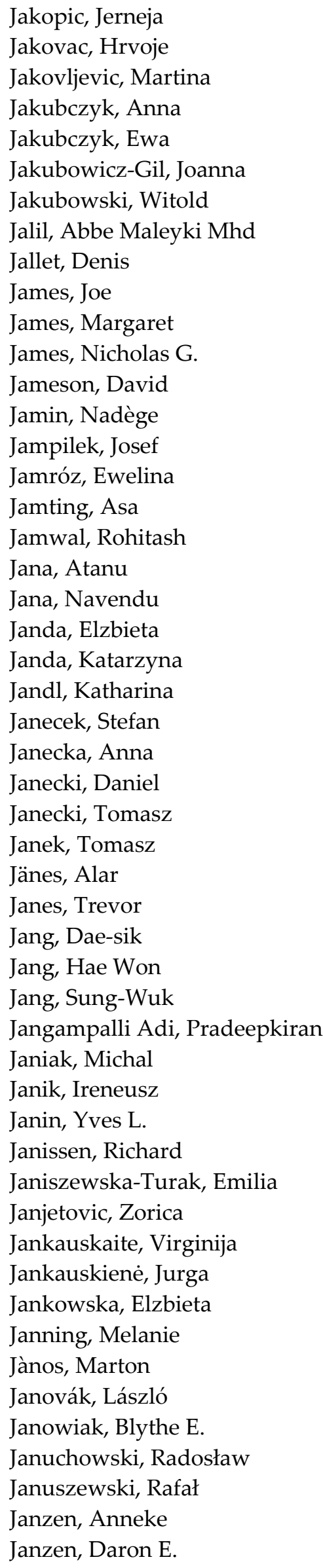

Japaridze, Aleksandre Jara Palacios, María José Jara, Paul Jaradat, Nidal Jarak, Ivana Jarczewski, Sebastian Jaren, Carmen Jarocka-Karpowicz, Iwona Jarończyk, Małgorzata Jaros, Doris Jarosz-Wilkołazka, Anna Jarzabek, Dariusz Jarzebski, Maciej Jasinski, Marcin Jasinski, Radomir Jasinski, Radoslaw Jasiurkowska-Delaporte, Małgorzata Jaśkowska, Jolanta Jastrzab, Renata Jastrzebska, Beata Jastrzebska, Izabella Jasutiene, Ina Jatkowska, Natalia Jaudzems, Kristaps Jaumot, Joaquim Jay, Steven Jayaprakash, Priyamvada Jazvinšćak Jembrek, Maja Jaźwiński, Jarosław Jean, Ludovic Jeandet, Philippe Jedelská, Jarmila Jedrejek, Dariusz Jędrzejczyk, Roman J. Jedziniak, Piotr Jee, JunGoo Jee, Jun-Pil Jeffrey, Kieft Jekle, Mario Jeldres, Ricardo I. Jeleń, Henryk Jeleń, Henryk H. Jeleń, Małgorzata Jelińska, Anna Jelliss, Paul Jelokhani-Niaraki, Masoud Jelonek, Katarzyna Jelsch, Christian Jena, Himanshu Sekhar Jenett-Siems, Kristina Jenkins, Ian 
Jenny, Titus A.

Jensen, Frank

Jensen, Lars R

Jensen, Mikael

Jensen, Svend Borup

Jenssen, Håvard

Jeon, Jongho

Jeon, Oju

Jeon, Sung Ho

Jeong, Euikyoung

Jeong, Jae Min

Jeong, Keun-Yeong

Jeong, Lak Shin

Jeong, Nak Cheon

Jeong, Seong Hoon

Jeong, Yeon-jun

Jeremić, Sanja

Jerez, Nancy

Jerković, Igor

Jerkowić, Igor

Jermakowicz-Bartkowiak, Dorota

Jeromel, Ana

Jerzykiewicz, Lucjan

Jeschek, Markus

Jesionek, Marcin

Jesson, David

Jeucken, Aike

Jezierska-Mazzarello, Aneta

Ji, Guozhao

Ji, Peng

Ji, Pengfei

Ji, Shaofei

Ji, Tengfei

Ji, Tianjiao

Ji, Xiangling

Jia, Li

Jia, Shang

Jia, Tony

Jia, Yanwei

Jia, Zhe

Jiang, Fuyi

Jiang, Jianxiong

Jiang, Jing

Jiang, Jiuxing

Jiang, Nan

Jiang, Xuefeng

Jiang, Xuntian

Jiang, Zaixing

Jiang, Zhen

Jiang, Ziwen

Jiao, Kui
Jibran, Rubina

Jijie, Roxana

Jimbo, Mitsuru

Jiménez Arellanes, Adelina

Jiménez Carvelo, Ana María

Jimenez Migallón, Alfonso

Jiménez, Jordi Juárez

Jiménez-Araujo, Ana

Jiménez-Aspee, Felipe

Jiménez-Barbero, Jesus

Jiménez-Jiménez, Félix Javier

Jiménez-Morillo, Nicasio Tomás

Jiménez-Tenorio, Manuel

Jimeno, Ciril

Jimi, Eijiro

Jin Cheol, Kim

Jin, Congrui

Jin, Fang

Jin, Guo-Xin

Jin, Jiefu

Jin, Min

Jin, Ning-de

Jin-chul, Kim

Jindřich, Jindřich

Jing, Hui

Jios, Jorge L.

Jiri, Pospisil

Jirkovsky, Eduard

Jishi, Radi

Jo, Hyunil

Jobe, Timothy

Jobic, Stephane

Jochum, Christoph

Jodłowski, Grzegorz

Johannissen, Linus $\mathrm{O}$.

John Jervis, Peter

John, Łukasz

John, Rohan

Johnson, Aaron

Johnson, Christopher

Johnson, Elizabeth

Johnson, Joshua

Johnson, Samuel A.

Johnson, Shelby L.

Johnsson, Mats

Johnston, Linda J.

Johnston, Michael J. W.

Johnstone, Jeanette

Jokić, Stela

Jonathan B., Cooper

Jones, Christopher E. 
Jones, Marjorie

Jonnalagadda, Sreekanth

Joó, Ferenc

Joo, Soo-Hyun

Joos, Jonas

Jordá, José Luis

Jordão, António Manuel

Jordheim, Monica

Jørgensen, Bodil

Jørgensen, Kåre B.

Jorrin-Novo, Jesus V.

Jose, Jiney

Joshi, Pushpa Raj

Joshi-Navare, Kasturi

Jovanović, Aleksandar

Jovanovic, Zivko

Joven, Jorge

Joyce, Paul

Jozic, Ivan

Jóźwicki, Wojciech

Juan, Maria Emilia

Juárez-Maldonado, Antonio

Judeh, Zaher

Judeinstein, Patrick

Juhasz, Bela

Julander, Justin G.

Julian, Fernando

Julien, Christian M.

Julien, Pierre

Jung De Andrade, Mônica

Jung, Byung Jun

Jung, Ho-Sup

Jung, Hye Jin

Jung, Hyunsook

Jung, Ji Hoon

Jung, Joohee

Jung, Min-Cherl

Jung, Seunho

Jung, Sokhee Philemon

Jung, Tae Sung

Jung, Woo-Jin

Jung, Yong Chae

Jung, Young Sung

Jungblut, Peter R.

Junichi, Kitanaka

Junior, Edeildo

Junkar, Ita

Juodkazis, Saulius

Juranović Cindrić, Iva

Jurasekova, Zuzana

Jurca, Titel
Jurczak, Janusz

Jurić, Slaven

Jurij, Trontelj

Jurikova, Tunde

Jurkonienè, Sigita

Jurkowska, Halina

Jurman, Giuseppe

Justicia, José

Juszczak, Lesław

Jyothi, Rajesh Kumar

K. Friestad, Gregory

K. Karabagias, Ioannis

Kaas, Quentin

Kabelác, Martin

Kabir, Abuzar

Kačániová, Miroslava

Kachrimanis, Kyriakos

Kącka-Zych, Agnieszka

Kaczmarek, Beata

Kaczmarek, Mariusz

Kaczmarek-Kędziera, Anna

Kaczor, Agnieszka A.

Kaddour, Hussein

Kadela-Tomanek, Monika

Kaden, Marika

Kadlubowski, Slawomir

Kadokawa, Jun-ichi

Kadota, Isao

Kafarski, Pawel

Kafouris, Demetris

Kageyama, Hakuto

Kagota, Satomi

Kahlert, Stefan

Kairys, Visvaldas

Kairytè, Agnè

Kaisarly, Dalia

Kaiser, Annette

Kaiser, Fabian

Kaji, Hiroshi

Kajimoto, Shinji

Kajitani, Takashi

Kajiwara, Takashi

Kajiyama, Satoshi

Kálai, Tamas

Kalantar-zadeh, Kourosh

Kalas, Wojciech

Kalayda, Ganna

Kalhapure, Rahul S.

Kalíková, Květa

Kalina, Jan

Kalinich, John 
Kalinin, Vladimir

Kalinowska Lis, Urszula

Kalinowska, Monika

Kalinski, Jarmo-Charles J.

Kalisz, Stanisław

Kaliva, Maria

Kaliyan, Nalladurai

Kalkum, Markus

Kallithraka, Stamatina

Kallitsis, Joannis K.

Kalló, Gergő

Kallscheuer, Nicolai

Kalluru, Poliraju

Kalmár, József

Kalogianni, Despina

Kalogiouri, Natasa

Kalogirou, Andreas

Kaloyanova, Dora V.

Kalušević, Ana

Kalwat, Michael A.

Kamal, Fadia Ali

Kamali, Saeed

Kamanina, Natalia

Kamath, Divya

Kamenov, George

Kameo, Hajime

Kamgang Nzekoue, Franks

Kamiloglu, Senem

Kaminska, Bozena

Kamińska, Joanna

Kaminski, Daniel M.

Kaminski, Kamil

Kamiński, Krzysztof

Kaminsky, Werner

Kamm, Birgit

Kamysz, Elżbieta

Kan, Chi-wai

Kanada, Masamitsu

Kanai, Masashi

Kananovich, Dzmitry G.

Kanaori, Kenji

Kanapitsas, Athanasios

Kanda, Naoko

Kandathil, Shaun M.

Kandil, Sahar

Kandylis, Panagiotis

Kaneko, Gen

Kanematsu, Hideyuki

Kang, Chen

Kang, Chulhun

Kang, Hee Eun
Kang, Jianming

Kang, Jun-Gill

Kang, Ju-Seop

Kang, Kyo Bin

Kang, Kyungsu

Kang, Kyungtae

Kang, Minjee

Kang, Nam Sook

Kang, Sang Sun

Kang, Sun Chul

Kang, Wonku

Kang, Yang Jun

Kang, Young-Hee

Kang, Yunqing

Kangas, Heli

Kangas, Teija

Kani, Kian

Kania, Henryk

Kano, Naoki

Kant, Sashi

Kantminienè, Kristina

Kantzas, Apostolos

Kao, Chai-Lin

Kao, Cheng-Yen

Kao, Peter

Kao, Shao-Hsuan

Kaplan, Barbara

Kappel, Noemi

Kappenstein, Charles

Kapusta, Ireneusz

Kar, Supratik

Karabagias, Ioannis

Karabaliev, Miroslav

Karagiannis, Tom

Karahan, H. Enis

Karakashev, Stoyan

Karakoti, Ajay

Karam, Sherif

Karaman, Maja

Karaman, Rafik

Karamanoli, Aikaterini

Karamaouna, Filitsa

Karasali, Helen

Karbownik, Agnieszka

Karcz, Dariusz

Karczmarzyk, Zbigniew

Kardassis, Dimitris

Kareiva, Aivaras

Karelson, Mati

Karikas, George-Albert

Karim, Nasiara 
Kariyat, Rupesh

Kårlund, Anna

Karnati, Konda Reddy

Karolewicz, Bożena

Károly, Lázár

Karousou, Eugenia

Karpe, Avinash V.

Karpichev, Yevgen

Karpinski, Tomasz M.

Karra, Laila

Karvelas, Evangelos

Karwowska, Małgorzata

Karwowski, Boleslaw

Kasapidou, Eleni

Kaseem, Mosab

Kaserer, Teresa

Kashchenko, Nina I.

Kashif, Muhammad

Kashman, Yoel

Kasiotis, Kostas

Kaškonienè, Vilma

Kasnatscheew, Johannes

Kašparovský, Tomáš

Kasperkiewicz, Paulina

Kasprowicz, Dobrosława

Kasprzak, Artur

Kaszowska, Marta

Kataev, Evgeny

Katahira, Rui

Katalinić, Maja

Katanić, Zorana

Kataoka, Hiroyuki

Kataoka, Naoyuki

Katarzyna, Malarz

Katayama, Takeshi

Kathuria, Himanshu

Katikou, Panagiota

Katin, Konstantin

Kato, Eisuke

Kato, Fumihiro

Kato, Keisuke

Kato, Koichi

Kato, Takamitsu

Katsafadou, Angeliki

Katselis, George

Katsila, Theodora

Katsuyama, Yohei

Kattamuri, Padmanabha

Katunin, Andrzej

Kauppinen, Anu

Kauppinen, Esko I.
Kaur, Amandeep

Kaur, Gunveen

Kaushik, Nagendra Kumar

Kavan, Ladislav

Kavita, Kumari

Kawabata, Kyuichi

Kawaguchi, Seigou

Kawaguchi, Shin-ichi

Kawai, Kiyohiko

Kawai, Takayuki

Kawakami, Akinori

Kawakami, Jun

Kawakami, Susumu

Kawakami, Yuki

Kawakita, Hidetaka

Kawamura, Fumio

Kawamura, Izuru

Kawanabe, Hiroshi

Kawano, Daniel Fábio

Kawato, Takayuki

Kawiak, Anna

Kayaki, Yoshihito

Kayano, Shin-ichi

Kazakova, Oxana B.

Kazimierski, Radosław

Kazokaite, Justina

Kazuhiro, Shikinaka

Kebukawa, Yoko

Keenan, Joanne

Keglevich, György

Kehr, Nermin Seda

Kelarakis, Antonios

Kele, Peter

Kellenberger, Stephan

Keller, Lena

Kellner, Stefanie

Kello, Martin

Kellogg, Joshua J.

Kem, William R.

Kenry, K.

Kerch, Garry

Kerman, Kagan

Kerr, William

Kerrigan, Nessan J.

Kerth, Andreas

Kertmen, Ahmet

Kerwin, Sean M.

Kesari, Kavindra Kumar

Kesharwani, Siddharth

Keshavarz, Meysam

Kęska, Paulina 
Ketkar, Amit

Kettler, Richard

Keyzers, Rob

Keyzers, Robert

Khaddaj Mallat, Rayan

Khalifa, Shaden

Khalili-Araghi, Fatemeh

Khallouki, Farid

Khalymbadzha, Igor A.

Khambu, Bilon

Khan, Abdul

Khan, Dr. Md. Shakhaoath

Khan, Hamidullah

Khan, Meraj Alam

Khan, Merajuddin

Khan, Mohammad Imran

Khan, Mohammad Sharif

Khan, Mujeeb

Khan, Omar F.

Khan, Sajid

Khan, Zafar K.

Khang, Gilson

Kharlampieva, Eugenia

Khattab, Tawfik

Khaw, Kooi Yeong

Khaw, Kooi Yepng

Khdair, Adnan

Khivantsev, Konstantin

Khlebnikov, Andrei I.

Khmaladze, Alexander T.

Khmelinskii, Igor

Khodadadi, Jay M.

Khoder, Mouhamad

Kholmukhamedov, Andaleb

Khong, Anthony

Khoshnood, Abbas

Khotimchenko, Maxim Y.

Khozin-Goldberg, Inna

Khung, Yit Lung

Khurana, Namrata

Khushwant Singh, Bhullar

Kia, Reza

Kicel, Agnieszka

Kichler, Antoine

Kiciński, Wojciech

Kiec-Kononowicz, Katarzyna

Kiel, Jaap

Kieliszek, Marek

Kierys, Agnieszka

Kierzek, Krzysztof

Kiesewetter, Dale O.
Kijewska, Monika

Kikuchi, Haruhisa

Kikuchi, Hidehiko

Kikuchi, Takeshi

Kikuta, Shingo

Kil, Kun-Eek

Kilbourn, Michael R.

Kilcawley, Kieran

Kilian, Krzysztof

Kilk, Kalle

Kim, Bonglee

Kim, Byoung Suhk

Kim, Choon Young

Kim, Chuk Young

Kim, Dong Seon

Kim, Dukmin

Kim, Gue-Hyun

Kim, Ha-Neui

Kim, Hee-Joon

Kim, Hoe Joon

Kim, Hojoong

Kim, Hong-Duck

Kim, Hoon

Kim, Hye Kyong

Kim, Hyonchol

Kim, Hyun Joon

Kim, Hyun Jung

Kim, Hyungsik

Kim, Hyun-Jung

Kim, Hyun-Soon

Kim, InKyeom

Kim, Jaehan

Kim, Jaehwan

Kim, Jaheon

Kim, Jangho

Kim, Jeong-Chul

Kim, Jeong-Hwan

Kim, Jeung Gon

Kim, Jihoon

Kim, Jinho

Kim, Jinseok

Kim, Jong-Ki

Kim, Jung-Hoon

Kim, Jungkwun

Kim, Ki Hyun

Kim, Kwangho

Kim, Kyoung Sub

Kim, Kyung Bo

Kim, Kyung Ho

Kim, Kyung-Min

Kim, Man-Hoe 


\begin{tabular}{|c|c|}
\hline Kim, Mi-hyun & Kishimoto, Shinji \\
\hline Kim, Min & Kishimura, Hideki \\
\hline Kim, Min Jung & Kiskin, Mikhail \\
\hline Kim, Min-Cheol & Kisková, Terézia \\
\hline Kim, Min-Soo & Kiss, Eva \\
\hline Kim, Pyung-Hwan & Kiss, Rita \\
\hline Kim, Sang Wan & Kitagawa, Seiichi \\
\hline Kim, Sanghoon & Kitagawa, Yuichi \\
\hline Kim, Seok-Ho & Kitagishi, Hiroaki \\
\hline Kim, Seung-Hyun & Kitajou, Ayuko \\
\hline Kim, Seungju & Kitamura, Akira \\
\hline Kim, Seyun & Kitamura, Yoshiaki \\
\hline Kim, Sok & Kitano, Yoshikazu \\
\hline Kim, Soo Un & Kitney, Stuart \\
\hline Kim, Soo-Kyung & Kiuru, Paula \\
\hline Kim, Sung-Hoon & Kiyama, Ryoiti \\
\hline Kim, Sung-Kun & Kiyokawa, Etsuko \\
\hline Kim, Tae Hwan & Kjaergaard, Magnus \\
\hline Kim, Tae Kon & Klajnert-Maculewicz, Barbara \\
\hline Kim, Woojin & Klar, Agnes \\
\hline Kim, Yang & Klaus, Anita \\
\hline Kim, Yongae & Kleczkowska, Patrycja \\
\hline Kim, Youngmee & Kleier, Daniel A. \\
\hline Kim, Young-Suk & Klein, Axel \\
\hline Kim, Yu-Jin & Klein, Mark A. \\
\hline Kimbaris, Athanasios & Klein, Susanne \\
\hline Kimura, Ken-ichi & Klejdus, Borivoj \\
\hline Kimura, Kiminori & Klem, Karel \\
\hline Kimura, Masanari & Klempová, Tatiana \\
\hline Kimura, Tetsunari & Klepac, Damir \\
\hline Kinder, David & Kleszczyński, Konrad \\
\hline Kindl, Marija & Klimek-Ochab, Magdalena \\
\hline King, Joan M. & Kline, Paul \\
\hline King, Neil P. & Klingeler, Rudiger \\
\hline Kingery, William L. & Kljusurić, Jasenka Gajdoš \\
\hline Kinzhalov, Mikhail A. & Klochkov, Sergey G. \\
\hline Kiokias, Sotirios N. & Klochkov, Vladimir V. \\
\hline Kippes, Nestor & Klokov, Dmitry \\
\hline Kirby, Christopher W. & Kloskowski, Tomasz \\
\hline Kirchmair, Johannes & Klouček, Pavel \\
\hline Kirin, Srecko & Kluczyk, Alicja \\
\hline Kirsch, Gilbert H. & Kluczyk-Korch, Katarzyna \\
\hline Kirsch, Stefan F. & Kmita, Angelika \\
\hline Kirubakaran, Palani & Knapp, Michael \\
\hline Kirwan, Jennifer & Knapp, Spencer \\
\hline Kiryukhantsev-Korneev, Ph V. & Knauth, Philippe \\
\hline Kiselev, Anton & Kňazovická, Vladimíra \\
\hline Kiselev, Vitaly & Knez, Damijan \\
\hline Kishida, Masao & Knežević, Anamarija \\
\hline Kishigami, Satoshi & Knezevic, Jelena \\
\hline Kishimoto, Jiro & Kniazeff, Julie \\
\hline
\end{tabular}


Knight, Christopher

Knittelfelder, Oskar

Knobloch, Thomas J.

Knölker, Hans-Joachim

Knudsen, Gabriel

Knupp, Gerd

Knuschke, Torben

Knutsen, Erik

Ko, Horng-Huey

Ko, Sung-kyun

Ko, Younhee

Kobayashi, Motoyoshi

Kobayashi, Rika

Kobayashi, Yayoi

Kobayashi, Yuichiro

Kobeissy, Firas

Kober, Kord

Kobrak, Mark N.

Kobus-Cisowska, Joanna

Kochmańska, Agnieszka

Kocira, Sławomir

Kockmann, Norbert

Kodama, Shintaro

Kodani, Sean D.

Koech, Phillip

Koel, Mihkel

Koenig, Mary Dawn

Koff, Andrew

Koga, Yoshikatsu

Koh, Eunmi

Koh, Gar Yee

Kohanbash, Gary

Köhl, Gudrun

Koikawa, Masayuki

Koike, Haruki

Koike, Mari

Koivisto, Janne

Koizume, Shiro

Kojdecki, Marek Andrzej

Kojima-Yuasa, Akiko

Kok, Victor C.

Kokado, Kenta

Kokina, Tatyana E.

Kokkarachedu, Varaprasad

Kokkinakis, Emmanouil

Kokkola, Tarja

Kokoska, Ladislav

Kokotos, George

Kolackova, Ivana

Kolakowski, Zbigniew

Kolar, Mitja
Kolaric, Branko

Kolesińka, Beata

Kolesnikova, Sophia A.

Kolev, Svetoslav

Kolling, Stefan

Kolmas, Joanna

Kolniak-Ostek, Joanna

Kolobov, Alexandr Alexandrovich

Kolocouris, Antonios

Kolodziejczyk, Joanna

Kołodziejczyk, Krzysztof

Kolodziejski, Pawel Antoni

Kolot, Mikhail N.

Kolukisaoglu, Üner

Komarnicka, Urszula K.

Komatsu, Satoshi

Komives, Tamas

Komorowska, Beata

Konar, Arkaprabha

Konar, Sumit

Konc, Janez

Kończak, Magdalena

Kondo, Jiro

Kondo, Mizuho

Kondo, Shin-ichi

Konev, Alexander

Kong, De-Ming

Kong, De-Xin

Kong, Duanyang

Kong, Fangong

Kong, L.X.

Kong, Lingyan

Kong, Won-Sik

Kong, Xian

Kong, Xiangrui

Konger, Raymond L.

Konieczny, Piotr

König, Gerhard

Königs, Rene M.

Konigsberg, William $\mathrm{H}$

Konishi, Hiroaki

Konishi, Masaaki

Konjevoda, Paško

Konno, Hiroyuki

Konno, Katsuhiro

Konno, Tomohiro

Könönen, Eija

Kononikhin, Alexey S.

Konopka, Iwona

Konrad, Futyma

Konrad, Lutz 
Konstantinos, Litinas

Konstantinov, Spiro M.

Kontek, Bogdan

Kontogiannidou, Eleni

Kontogiorgis, Christos

Kontominas, Michael G.

Kontoni, Denise-Penelope N.

Kontoravdi, Cleo

Kooistra, Albert

Kopaczyńska, Marta

Kopeć, Aneta

Kopecký, Vladimír

Kopel, Pavel

Kopjar, Mirela

Kopjar, Nevenka

Kopke De Aguiar, Jair Adriano

Kopra, Kari

Kopsahelis, Nikolaos

Kopustinskiene, Dalia M.

Korach-André, Marion

Korb, Marcus

Korbecki, Jan

Korchowiec, Beata

Kordyukova, Larisa

Kordyum, Elizabeth

Kore, Rajkumar

Korlyukov, Alexander A.

Kormos, Atilla

Kornhuber, Malte.

Korolkov, Ilya V.

Korolkova, Yuliya

Koronkiewicz, Stanisława

Koroteev, Pavel S.

Korshun, Vladimir A.

Korte, Carsten

Kortholt, Arjan

Koseva, Neli

Kosik-Bogacka, Danuta

Kosior, Dominik

Kosma, Paul

Kosmala, Monika

Košmerl, Tatjana

Košmrlj, Janez

Kosmulski, Marek

Košnář, Zdeněk

Kosobucki, Przemysław

Kosova, Nina

Kostakis, George

Kostakis, Ioannis

Kostareva, Anna

Kostecka, Malgorzata
Kostecka-Gugala, Anna

Kostecki, Marek

Kostevšek, Nina

Kostić, Aleksandar Ž.

Kostikov, Alexey P.

Kostoulias, Xenia

Kosyakov, Dmitry S.

Koszegi, Tamas

Kőszegi, Tamás

Koszelewski, Dominik

Koszelnik, Piotr

Kotek, Jan

Kotlyar, Alexander B.

Kotlyar, Margarita V.

Kotora, Martin

Kotra, Lakshmi

Kotra, Lakshmi P.

Kotsiantis, Sotiris

Kotsuchibashi, Yohei

Koubogiannis, Dimitrios G.

Koudounas, Konstantinos

Koufaki, Maria

Koumoulos, Elias P.

Kouretas, Dimitrios

Koutahzadeh, Negin

Koutelidakis, Antonios E.

Koutentis, Panayiotis A.

Koutsogiannis, Ioannis

Kouznetsova, Valentina

Kovačević Ganić, Karin

Kovačević, Borislav

Kovačević, Davor

Kovačević, Strahinja

Kovacheva, Daniela

Kovacs, Lajos

Kovacs, Richard

Kovács, Zoltán

Koval, Alexey

Kovalenko, Sergey

Kovalevsky, Andrei V.

Kovar, Petr

Kováts, Éva

Kovbasnjuk, Olga

Kowal, Krzysztof

Kowalczewski, Przemysław

Kowalczuk, Dorota

Kowalczyk, Aleksandra

Kowalczyk, Dariusz

Kowalczyk, Iwona

Kowalczyk, Mariusz

Kowalczyk, Tim 
Kowalczyk, Tomasz

Kowalska, Ewa

Kowalska, Iwona

Kowalska, Joanna

Kowalska, Karolina

Kowalska, Katarzyna

Kowalski, Konrad

Kowalski, Radosław

Kowol, Christian R.

Kowolik, Claudia

Koyack, Marc

Koyioni, Maria

Kozak, Yoram

Kozanecki, Marcin

Kozempel, Ján

Koziak, Katarzyna

Kozieł, Edmund

Koziel, Jacek

Koziol, Mateusz

Koziróg, Anna

Koźlecki, Tomasz

Kozlevčar, Bojan

Kozliak, Evguenii I.

Kozlík, Petr

Kozlov, Mikhail V.

Kozlov, Sergey

Kozlova, Ekaterina

Kozlova, Elena

Kozlowska, Justyna

Kozłowska, Mariola

Koźmiński, Wiktor

Kragović, Milan

Krajka-Kuźniak, Violetta

Krakhalev, Mikhail N.

Krakowiak, Agnieszka

Krakowiak, Joanna

Královec, Karel

Kranz, Mathias

Krasavin, Mikhail

Krasikova, Raisa N.

Krasnoslobodtsev, Alexey V.

Kratky, Lukas

Krátký, Martin

Kratochvilova, Irena

Kraus, Christoph

Krause, Frank

Krause, Günter

Krawczyk, Joanna

Krchnak, Viktor

Kreis, Wolfgang

Krela-Kaźmierczak, Iwona
Křen, Vladimir

Krenn, Liselotte

Kressler, Jörg

Kreuter, Justin

Krga, Irena

Kriegsmann, Jörg

Krikstolaityte, Vida

Krisch, Judit

Krishna, Gokul

Krishnakumar, Shyam

Krishnan, Krish

Krissinel, Eugene

Kristensen, Tor Erik

Krisyuk, Vladislav

Kritikos, Georgios

Kritsi, Eftichia

Krivoruchko, Anastasiia

Kroeker, S.

Krokidis, Marios

Krokosz, Anita

Król, Ewelina

Królicka, Agnieszka

Kronekova, Zuzana

Kručaitè, Gintarè

Krueger, Joanna K.

Kruger, Cherie

Kruger, Jacob S.

Krukiewicz, Katarzyna

Krumkacheva, Olesya A.

Krummenacher, Claude

Krumova, Sashka

Kruse, Martin

Kruszynski, Rafal

Krysinski, Pawel

Kryštof, Vladimír

Kryštofová, Svetlana

Krystyna, Skalicka-Woźniak

Krzeminski, Marek

Krzywiecki, Maciej

Krzyżak, Artur Tadeusz

Ksenofontov, Alexander

Kshirsagar, Prakash G.

$\mathrm{Ku}$, Kang-Mo

$\mathrm{Ku}$, Seockmo

Kuan, Yu-Hsiang

Kubala, Lukáš

Kubatka, Peter

Kubatzky, Katharina

Kubica, Paweł

Kubicek, Christian Peter

Kubíček, Vojtěch 
Kubíčková, Anna

Kubina, Robert

Kubinová, Renata

Kubinyi, Miklós

Kubissa, Wojciech

Kubo, Anna-Liisa

Kubo, Atsushi

Kubo, Masataka

Kubo, Miwa

Kubo, Takanori

Kubo, Yoshinao

Kuca, Kamil

Kuča, Kamil

Kučera, Lukáš

Kučera, Tomáš

Kučerik, Jiří

Kucerova, Marta

Kucharska, Karolina

Kucinska, Malgorzata

Kuckling, Dirk

Kućko, Agata

Kucuk, Israfil

Kuczera, Krzysztof

Kudasik, Mateusz

Kuddannaya, Shreyas

Kúdela, Jozef

Kuder, Tomasz

Kudłak, Błażej

Kudo, Fumitaka

Kudo, Yuta

Kudr, Jiri

Kudryavtsev, Denis S.

Kuehne, Irina

Kueppers, Stephan

Kues, Wilfried A.

Kühl, Anja A.

Kühn, Oliver

Kujawska, Małgorzata

Kujawski, Jacek

Kujawski, Wojciech

Kukli, Kaupo

Kukovec, Boris-Marko

Kukula-Koch, Wirginia

Kula, Karolina

Kula, Sławomir

Kula-Pacurar, Anna

Kulbacka, Julita

Kulczyński, Bartosz

Kulda, Vlastimil

Kulichikhin, Valery

Kulkarni, Jayesh A.
Kulkarni, Shashank

Kulma, Magdalena

Kulnitskiy, Boris A.

Kulozik, Ulrich

Kulp, Samuel

Kumagai, Yuya

Kumal, Raju

Kumamoto, Eiichi

Kumar Patel, Sravan

Kumar, Anuj

Kumar, Dhiraj

Kumar, Dushyant

Kumar, Hirdesh

Kumar, Keshav Senthil

Kumar, Krishan

Kumar, Majeti N.V. Ravi

Kumar, Manu

Kumar, Naresh

Kumar, Nirbhay

Kumar, Pankaj

Kumar, Penmetcha

Kumar, Pradeep

Kumar, Ravi

Kumar, Santosh

Kumar, Shailabh

Kumar, Shailesh

Kumar, Sujeet

Kumar, Suneel

Kumar, Vijay

Kumi-Diaka, James

Kun, Li

Kuncewicz, Joanna

Kung, Chung-Wei

Kung, Woon-Man

Kunji, Edmund R.S.

Kunsági-Máté, Sándor

Kunz, Horst

Kunz, Wolfram

Kuo, Chia-Hung

Kuo, Dong-Hau

Kuo, Meng I.

Kuo, Ping-Chung

Kuo, Shiaowei

Kuo, Tsung-Rong

Kuo, Wen-Ten

Kuo, Yao-Haur

Kupka, Teobald

Kuppusamy, Senthil Kumar

Kurańska, Maria

Kurasaki, Masaaki

Kurc, Beata 
Kurczab, Rafał

Kurdziel, Magdalena

Kurien, Biji T.

Kurihara, Hideyuki

Kuroboshi, Manabu

Kuroda, Daisuke

Kuroda, Takayoshi

Kuroiwa, Keita

Kurreck, Prof Jens

Kursula, Petri

Kurtán, Tibor

Kurtenbach, Stefan

Kurzatkowska, Katarzyna

Kurzawa, Marzanna

Kusaczuk, Magdalena

Kustov, Leonid

Kustrin, Snezana

Kustrowski, Piotr

Kusukawa, Takahiro

Kutner, Wlodzimierz

Kuttel, Michelle

Kuttner, Christian

Kuwabara, Takuya

Kuwajima, Kunihiro

Kuwatani, Tatsu

Kuzhir, Polina

Kuzmin, Alexei

Kuznetsov, Maxim L.

Kuznetsov, Nikita

Kuźnik, Nikodem

Kuzuhara, Daiki

Kuzuya, Akinori

Kvasnica, Miroslav

Kwak, Jong Hwan

Kwak, Seonyeong

Kwan, Hiu-yee

Kwaśniewska, Anita

Kwasniewski, Misha T.

Kwiecień, Iwona

Kwok, Hang Fai

Kwok, Henry Hang Fai

Kwon, Hyuk-Jun

Kwon, Hyung Joo

Kwon, Jaeyul

Kwon, Jung-Hwan

Kwon, Seong Jung

Kwon, So Hee

Kwon, Soon-Hong

Kwon, Sung Won

Kwon, Sunoh

Kwon, Yongchan
Kwon, Young M.

Kwon, Youngjoo

Kwon, Young-Wan

Kyoungsik, Chang

Kyriakopoulou, Konstantina

Kyzas, George

Kyzas, George Z.

Kyzioł, Agnieszka

L. Pey, Angel

L. Waters, Marcey

L'homme, Laurent

La Clair, James

La Flor, Silvia De

La Foresta, Fabio

La Motta, Concettina

La Penna, Giovanni

La Regina, Giuseppe

La Rosa, Carmelo

La Spina, Martina

Labbé, Simon

Labbozzetta, Manuela

Labrou, Nikolaos

Lacaille-Dubois, Marie-Aleth

Lacal, Juan Carlos

Lachenmeier, Dirk

Lachman, Jaromir

Lachowicz, Sabina

Lachter, Elizabeth R.

Lackie, Peter M.

Lacko, Andras

Lackovic, Igor

Lacomme, Christophe

Łączkowski, Krzysztof Z.

Ladavos, Athanasios K.

Lade, Harshad

Lafond, Mickaël

Lafont, René

Lafrenie, Robert M.

Laganà, Antonio Simone

Laghi, Luca

Lago, Ana B.

Lago, João Henrique Ghilardi

Lagoa, Ricardo

Lagoumintzis, George

Lagravère, Manuel $\mathrm{O}$.

Lai, Chian-Hui

Laitinen, Riikka

Laitinen, Risto

Lakatos, Boris

Lakowski, Ted

Lakshmanan, Imayavaramban 
Lalas, Stavros

Lalatsa, Aikaterini

Lam, Kevin

Lam, Sio-Hong

Lama, Adriano

Lamac, Martin

Lamari, Fotini N.

Lamas, Alexandre

Lambert, Joerg

Lambert, Karen

Lambertucci, Catia

Lambri, Osvaldo Agustín

Lambrinidis, George

Lambruschini, Chiara

Lamers, Christina

Lamichhane, Rajan

Lamouroux, Emmanuel

Lamponi, Stefania

Lamprou, Dimitrios A.

Lamuta, Caterina

Lamy De La Chapelle, Marc

Lamy, Elsa

Lamy, Evelyn

Lan, Yucheng

Lanças, Fernando

Lanceros-Mendez, Senentxu

Landon, Chelsea Dawn

Lange, Heiko

Langelaan, David N.

Langendorf, Christopher

Langer, Thierry

Langhansova, Lenka

Laparra, Jose Moisés

Lapcík, Oldrich

Łapiński, Andrzej

Lapteva, Maria

Laraia, Luca

Lara-Sanchez, Agustin

Laratta, Bruna

Laricchiuta, Annarita

Larin, Sergey

LaRocque, Jan

Laronze-Cochard, Marie

Larraneta, Eneko

Larson, Åsa

Larson, Nicholas B.

Lartigue, Lenaic

Lasfar, Ahmed

Lash, Timothy D.

Laskowska, Magdalena

Laskowski, Łukasz
Lasseuguette, Elsa

Lassi, Ulla

Latek, Dorota

Latella, Giovanni

Łatka, Leszek

Latorre-Moratalla, Mariluz Luz

Latowski, Dariusz

Lattorff, H. Michael G.

Lattuada, Marco

Lau, Chungho

Laube, Markus

Lauberts, Maris

Läuchli, Severin

Laudadio, Emiliano

Lauersen, Kyle

Laughton, Charles A.

Lauren E., Brown

Laurenti, Enzo

Laurini, Erik

Lavela, Pedro

Lavilla, Rodolfo

Lavine, Barry

Lavoie, Serge

Law, Ruby

Ławniczak, Łukasz

Laws, Edward A.

Lay, Horng-Liang

Lazarides, Theodore

Lazzara, Giuseppe

Lazzarato, Loretta

Lazzari, Massimo

Lazzaroni, Roberto

Le Borgne, Marc

Le Gall, Gwenaelle

Le Grognec, Erwan

Le Ouay, Benjamin

Le Pogam, Pierre

Le Quéré, Jean-Luc

Le Questel, Jean-yves

Le Roes-Hill, Marilize

Le Thanh-Blicharz, Joanna

Le, T. T. Yen

Leabu, Mircea

Leadbeater, Nicholas

Leahu, Ana

Leal, Ana Mendes

Leao, José

Leardini, Fabrice

Lea-Smith, David

Leavesley, David

Lebeau, Bénédicte 
Lebedeva, Olga E.

Lebedyeva, Iryna

Leber, Bettina

Lebreton, Jacques

Lebron, José Antonio

Lebrun, Vincent

Lecamp, Laurence

Lech, Krzystof

Lech, Krzysztof

Lechel, Andre

Lecian, Orchidea Maria

Leclercq, Loic

Lecomte, Philippe

Lederkremer, Gerardo Z.

Ledesma, Ana Estela

Ledo, Ana

Lee, Chang Hoon

Lee, Changgu

Lee, Che-Hsin

Lee, Cheng-I

Lee, Chia-Hung

Lee, Chiang-Wen

Lee, Chien-Hsing

Lee, Ching-Kuo

Lee, Chong

Lee, Choong Hwan

Lee, Chun-Lin

Lee, Dae Young

Lee, Dae-Hee

Lee, Do Yup

Lee, Donghyun

Lee, Dong-ki

Lee, Dongkyu

Lee, Dong-Un

Lee, Han Eol

Lee, Hee Jae

Lee, Hon Man

Lee, Hong-Ki

Lee, Huei-Jane

Lee, Hwa-Jin

Lee, Hye Suk

Lee, Hyun

Lee, Ik Jae

Lee, I-Ta

Lee, Jaehwi

Lee, Jeongmi

Lee, Jetty Chung-Yung

Lee, Jihyun

Lee, Jin Hyung

Lee, Jin-Koo

Lee, Jin-Kyun
Lee, Jiunn Haw

Lee, Ji-Woong

Lee, Jong-Dae

Lee, Joon Kyu

Lee, Jue-Yeon

Lee, Jun Sik

Lee, Jung Ro

Lee, Jung Seok

Lee, Junseong

Lee, Ki-Teak

Lee, Kun-Mu

Lee, Kyungmin

Lee, Li-Ting

Lee, Meng-shiou

Lee, Min Wook

Lee, Mina

Lee, Sang Gil

Lee, Sang Kook

Lee, Sang-Han

Lee, Sang-hun

Lee, Sejoon

Lee, Seung Jae

Lee, Seunggu

Lee, Sheng-Long

Lee, Shiow-Ju

Lee, Shyi-Long

Lee, Sukmook

Lee, Sung Keun

Lee, Sungyul

Lee, Tae Jin

Lee, Tse-Min

Lee, Tzu-Tai

Lee, Victor

Lee, Woo-Kyung

Lee, Yeon-Ju

Lee, Yongbok

Lee, Yu-Hsuan

Lee, Yun-Sang

Lee-Ruff, Edward

Lees, Justin

Leeth, Caroline

Lefèvre, Guillaume

Lefèvre, Thierry

Leffler, Hakon

Legaz González, María Estrella

Léger, Bastien

Legon, Anthony

Legrand, François-Xavier

Legrand, Vincent

Legros, Julien

Leherte, Laurence 
Lehnherr, Dan

Lehocky, Marian

Lehtonen, Ari

Lei, Ting

Lei, Zhentian

Leidenheimer, Nancy

Leisz, Sandra

Leitão, Jorge H.

Leitao, Ruben Elvas

Leitinger, Birgit

Lekka, Malgorzata

Lelešius, Raimundas

Lelli, Moreno

Lemaire, Martin

Lemanowicz, Marcin

Lemercier, Gilles

Lemli, Beata

Lemordant, Daniel

Len, Christophe

Lenaic, Couedel

Lenardao, Eder

Lenaz, Giorgio

Lenci, Elena

Lenhard, Justin R.

Lennartz, Michelle

Lentini, Giovanni

Lentz, Christian

Lenucci, Marcello Salvatore

Lenz, Christof

Lenzi, Marcelo

Leon Serrano, Javier

Leon, Francisco

León, Josefa

Léonard, Estelle

Leonard, Stephen

Leondiadis, Leondios

Leonelli, Francesca

Leong, David T.

Leong, Max K.

Leoni, Alberto

Leonidas, Demetres D.

Leonidas, Palilis

Leonowicz, Zbigniew

León-Rivera, Ismael

Leontopoulos, Stefanos

Leopold, Loredana

Leow, Melvin Khee-Shing

Lepareur, Nicolas

Lephart, Edwin D.

Leporatti, Stefano

Lepore, Maria
Leporini, Mariarosaria

Leprince-Wang, Yamin

Lequin, Olivier

Les, Francisco

Lesgards, Jean-François

Leshchenko, Elena V.

Leśniewicz, Anna

Lešnik, Samo

Lesnikowski, Zbigniew J.

Lesov, Ivan

Lessard, Benoit

Lesyk, Roman B.

Lete, Esther

Letek, Michal

Leung, Chung-Hang

Leung, Ivanhoe

Leung, KaHo

Leutou, Alain Simplice

Levchenko, Igor

Leveneur, Jerome

Levente Nagy, Csaba

Lević, Steva

Levina, Aviva

Levine, Mindy

Levy, Mel

Levy-Ontman, Oshrat

Lewandowicz, Grażyna

Lewandowska, Katarzyna

Lewandowski, Wiktor

Lewin, Matt

Lewinska, Anna

Lewis, Phillip

Lewkowicz, Aneta

Leyva-Jimenez, Francisco Javier

Lezot, Frederic

Lezsovits, Ferenc

Lhiaubet, Virginie Lyria

Li, Aifeng

Li, Bing-Yan

Li, Chengxi

Li, Dong

Li, Dongmei

Li, Feng

Li, Fenghua

$\mathrm{Li}, \mathrm{Fu}$

Li, Fu-shuang

Li, Gang

Li, Guangming

Li, Guanna

Li, Guohui

Li, Haolong 


\begin{tabular}{|c|c|}
\hline $\mathrm{Li}, \mathrm{He}$ & Li, Xunde \\
\hline Li, Hongda & Li, Yan \\
\hline Li, Hou-Jin & Li, Yang \\
\hline Li, Hsing-Hui & Li, Yaozong \\
\hline $\mathrm{Li}, \mathrm{Hu}$ & Li, Ying \\
\hline Li, Hua & Li, Yiwen \\
\hline Li, Huabin & Li, Yuanzuo \\
\hline Li, Hui & Li, Yubin \\
\hline Li, Jianfeng & Li, Yunguo \\
\hline Li, Jianmei & Li, Yuxin \\
\hline Li, Jiao Jiao & Li, Zhaohui \\
\hline Li, Jingan & Li, Zhengqiang \\
\hline Li, Jingpeng & Li, Zhi \\
\hline Li, Jinpeng & Li, Zhi-Minhg \\
\hline Li, Jinyu & Li, Zhiyu \\
\hline Li, Junjie & Liagre, Bertrand \\
\hline Li, Junrong & Liakos, Ioannis \\
\hline Li, Kefeng & Liang, Chen \\
\hline Li, Kuo-Tseng & Liang, Hao \\
\hline Li, Lan & Liang, Haozhao \\
\hline $\mathrm{Li}, \mathrm{Li}$ & Liang, Pi-Hui \\
\hline Li, Lianbo & Liang, Po-Huang \\
\hline Li, Liya & Liang, Wenyan \\
\hline Li, Mengxing & Liang, Xianrui \\
\hline Li, Ming-Yu & Liang, Yong \\
\hline Li, Mingzhong & Liang, Yuerong \\
\hline Li, Po-Hsien & Liang, Zhibin \\
\hline Li, Qian-Sheng & Liao, Hsiao-Wei \\
\hline Li, Qun & Liao, Jiunn-Der \\
\hline Li, Rachel & Liao, Jyh-fei \\
\hline Li, Ronghui & Liao, Ling-Hsiu \\
\hline Li, Ruei-Nian & Liao, Pan \\
\hline Li, Siheng & Liao, Peng \\
\hline Li, TaoTao & Liao, Shu-Chuan \\
\hline Li, Tiangang & Liao, Wupeng \\
\hline Li, Tianhu & Liao, Yi-Hung \\
\hline Li, Wei & Liao, Ying-Chih \\
\hline Li, Wen-Wu & Liao, Yonghong \\
\hline Li, Wenyi & Liaudanskas, Mindaugas \\
\hline Li, Wenyue & Liaw, Chih-Chuang \\
\hline Li, Xian & Liberto, Erica \\
\hline Li, Xiang-Guo & Licata, Patrizia \\
\hline Li, Xianglin & Licea, Alexei \\
\hline Li, Xiangmei & Licini, Caterina \\
\hline Li, Xiangnan & Lickiss, Paul \\
\hline Li, Xiao & Lidon, Fernando \\
\hline Li, Xiaojun & Liebman, Joel \\
\hline Li, Xiaotao & Lieder, Barbara \\
\hline Li, Xinmin & Liefeith, Klaus \\
\hline Li, Xinming & Ligaray, Mayzonee \\
\hline Li, Xue & Ligat, Gaetan \\
\hline
\end{tabular}


Ligor, Magdalena

Liguori, Angelo

Likhacheva, Anna Yu

Likodimos, Vlassios

Liktor-Busa, Erika

Lim, Eun-Kyung

Lim, Jason

Lim, Ji-Hong

Lim, Jiseok

Lim, Ki-Taek

Lim, Kyu

Lim, Lee Wei

Lim, Lina H. K.

Lim, Sang Moo

Lim, Soyeon

Lima Do Monte-Neto, Rubens

Lima Ribeiro, Marcelo

Lima, Beatriz

Lima, Dênis

Lima, Raquel T.

Lima, Rui A.

Lima, Sofia

Limauro, Danila

Limbeck, Andreas

Limongi, Tania

Lin, Cheng-Wen

Lin, Cherng-Yuan

Lin, Chih-Hsun

Lin, Guiting

Lin, Haishu

Lin, Ho

Lin, Hung-Yin

Lin, Jer-An

Lin, Kuo-Shyan

Lin, Ligen

Lin, Long-Liu

Lin, Mei-Hsiang

Lin, Meng

Lin, Po-Heng

Lin, Qishan

Lin, Ruibiao

Lin, Ruwei

Lin, Shih-Yi

Lin, Shuo

Lin, Tung-Yi

Lin, Tzer-Bin

Lin, Weifeng

Lin, Yangju

Lin, Yang-wei

Lin, Yibin

Lin, Yingbo
Lin, Ying-Chi

Lin, Zheshuai

Lin, Zhiwei

Lin, Zhoumeng

Lin, Zongtao

Linares, Jose

Linares, María

Linares-Pastén, Javier A.

Lincoln, Richard

Lindahl, Ulf

Lindberg, Gerrick

Linder, Markus

Lindner, Martina

Lindquist, Nathan

Linert, Wolfgang

Link, Andreas

Linko, Veikko

Linseman, Daniel

Linul, Emanoil

Lionetti, Vincenzo

Lionetto, Maria Giulia

Liou, Gunn-Guang

Liou, Ying-ming

Lipert, Anna

Lipiński, Piotr F. J.

Lipparini, Filippo

Lippens, Guy

Lips, Katrin

Lipscomb, Michael W.

Lis Arias, Manuel J.

Liscano, Yamil

Lisec, Jan

Lisi, Lucia

Liška, Marek

Lisuzzo, Lorenzo

Litvić, Mladen

Liu, Bing-Lan

Liu, Bo

Liu, Chang

Liu, Chao-Lin

Liu, Chaoxing

Liu, Chengmei

Liu, Chen-Guang

Liu, Cheuk Lun

Liu, Chung-Chiun

Liu, Chunming

Liu, Dongxia

Liu, Dongxu

Liu, Fufeng

Liu, Fuguo

Liu, Gang 
Liu, Hai-yang

Liu, Hongbing

Liu, Hongde

Liu, Hongyu

Liu, Hongzhi

Liu, Ji

Liu, Jingjing

Liu, Jinming

Liu, Juewen

Liu, Jun

Liu, Jun-Jen

Liu, Kun

Liu, Liangliang

Liu, Maning

Liu, Miaomiao

Liu, Minmin

Liu, Pengda

Liu, Pengzhan

Liu, Shih-Jen

Liu, Shing-Hwa

Liu, Shiuh-Tzung

Liu, Shi-Xia

Liu, Shuang

Liu, Shyh-Jiun

Liu, Taihong

Liu, Tao

Liu, Xianhu

Liu, Xin

Liu, Xin-Yong

Liu, Xuqing

Liu, Yang

Liu, Yangyang

Liu, Yanrong

Liu, Yi-Hung

Liu, Ying

Liu, Yingjun

Liu, Yongliang

Liu, Yongqiang

Liu, Youzhong

Liu, Yu

Liu, Yue

Liu, Yung-Chuan

Liu, Yu-Peng

Liu, Zhaomin

Liu, Zhaopeng

Liu, Zhenqiu

Liu, Zhi

Liu, Zhiqian

Liu, Zitong

Livaniou, Evangelia

Liwinienko, Grzegorz
Liwo, Adam

Lizardi-Mendoza, Jaime

Lizcano, Fernando

Ljubenkov, Ivica

Llanos, Jaime

Llata, Marta Ruiz

Llop, Jordi

Llorca, Jordi

Lloveras, Vega

Lluna, Amparo Gamero

Lo Faro, Maria Josè

Lo Giudice, Angelina

Lo Sardo, Federica

Lo Scalzo, Roberto

Lo, Huang-Mu

Lo, Szecheng

Lo, Yi-Chen

Løbner-Olesen, Anders

Lobo, Glenn P.

Lobón, Natividad Chaves

Locatelli, Marcello

Lockridge, Oksana

Lodi, Alessia

Łodyga-Chruścińska, Elżbieta

Lodygin, Evgeny

Loginov, Dmitriy

Lognay, Georges

Lognay, Georges C.

Lohning, Anna

Loira, Iris

Loiseau, Philippe

Loizzo, Monica R.

Loizzo, Monica Rosa

Lojou, Elisabeth

Lok, Chun Nam

Lollo, Giovanna

Lomberget, Thierry

Lomonaco, Diego

Lomonaco, Tommaso

Lončar, Mirela Baus

Lončar, Nikola

Lončarević-Vasiljković, Nataša

Londergan, Casey

Londoño López, Martha Elena

Long, Brian

Long, Yuchen

Longhi, Giovanna

Longo, Angela

Longo, Maria A.

Longo, Pasquale

Longo, Sandro 
Longo, Valentina

Lönnberg, Harri

Loontjens, Ton J. A.

Lopes Teixeira, Gerson

Lopes, Adelino V.

Lopes, Ana

Lopes, Francisca

Lopes, Graciliana

López Nicolás, Jose Manuel

Lopez Rodilla, Jesus M.

López Romero, Juan M.

López, Concepción

Lopez, Jose Cristobal

López, Kelly Johana Figueroa

López, Lluvia

Lopez, Victor

Lopez-Camarillo, Cesar

López-Cornejo, Pilar

Lopez-Jaramillo, Francisco Javier

Lopez-Linares, Francisco

López-Linares, Juan Carlos

López-López, José A.

López-Lorente, Ángela Inmaculada

López-Salas, Nieves

Lopez-Serrano, Joaquin

Lopez-Viñas, Eduardo

Loppinet, Benoit

Łopusiewicz, Łukasz

Lordan, Ronan

Lorenc-Koci, Elżbieta

Lorentz, Axel

Lorenzetti, Stefano

Lorenzo, Jose M.

Lorenzo, Julia

Lorenzo-Morales, Jacob

Loret, Erwann P.

Loretz, Brigitta

Lorite, Pedro

Lorkiewicz, Pawel K.

Łoś, Szymon

Losada-Perez, Patricia

Losantos, Raúl

Loschenov, Victor B.

Losurdo, Giuseppe

Lotina-Hennsen, Blas

Lou, Chenguang

Lou, Hongxiang

Louarn, Essyllt

Louda, Petr

Louie, Stacey

Lourenço, Ana
Lourenço, Anália

Lourenço, Gustavo Jacob

Louvaris, Zafeiris

Love, Jason

Loveridge, Joel

Low, Nicholas

Lowell, Andrew

Lowery, Jonathan

Loza-Coll, Mariano Andres

Loza-Mejía, Marco A.

Loza-Mejía, Marco Antonio

Lozano, Jose Manuel

Lozano-Gorrin, Antonio D.

Lozinsky, Vladimir

$\mathrm{Lu}$, Chih-Hao

Lu, Chi-Yu

Lu, Chung-kuang

Lu, Jianqin

Lu, Jingzhi

Lu, Mei-Chin

$\mathrm{Lu}$, Meng

Lu, Pengtao

Lu, Qingyi

Lu, Quanlong

$\mathrm{Lu}$, Shaoyong

Lu, Shuiyu

Lu, Wenchao

Lu, Xiao

Lu, Xuejun

$\mathrm{Lu}, \mathrm{Yi}$

Lu, Yujiao

$\mathrm{Lu}$, Yuyun

Lu, Zhan-Hui

Lubawy, Jan

Lubin-Germain, Nadège

Lubowiecka, Izabela

Lucaciu, Constantin Mihai

Lucarini, Simone

Lucas, Andrew T.

Lucas, Guilherme

Lucas, Heather R.

Lucena, Rafael

Lucenti, Elena

Luchini, Claudio

Lucía Z., Flores-López

Lučić, Bono

Łuczaj, Wojciech

Luddi, Alice

Ludwiczuk, Agnieszka

Lugnier, Claire

Lugo, Jesus Eduardo 
Lui, Edmund Man King

Luís, Ângelo

Luis, Santiago V.

Luisetto, Roberto

Luisi, Renzo

Luiz Dotto, Guilherme

Lujanienè, Galina

Lukáč, Miloš

Lukasik, Rafal

Łukasz, Marczak

Lukaszewicz, Marcin

Lukaszkowicz, Krzysztof

Luke, Whiley

Lukić, Igor

Lukinac Čačić, Jasmina

Lukinac, Jasmina

Lukinavicius, Grazvydas

Lukšič, Miha

Lukyanov, Pavel A.

Luliński, Sergiusz

Luna Maldonado, Aurelio

Luna Moreno, Donato

Luna, Diego

Lundin, Jeffrey G.

Lung, Shiu Cheung

Lungu, Cristian

Lunov, Oleg

Lunter, Dominique

Luo, Danmeng

Luo, Hongliang

Luo, Jin

Luo, Kui

Luo, Rongcong

Luo, Wentai

Luo, Xiaomei

Luo, Yanhua

Luo, Zisheng

Lupan, Alexandru

Luppi, Barbara

Lupu, Stelian

Lusczek, Elizabeth R.

Lushchekina, Sofya

Łuszczki, Jarogniew

Lutfy, Kabirullah

Lutter, Erika

Lutz, Martin

Luu, Hung N.

Luz, Rita De Cássia Silva

Luzi, Francesca

Luzina, Olga

Luzzio, Fred
Lv, Kangle

Lvova, Larisa

Lyashchenko, Serge

Łyczakowski, Jan

Łyczko, Jacek

Lykidis, Charalampos

Lyon, R. E.

Łyszczek, Renata

Lyu, Hailong

Lyukmanova, Ekaterina N.

M. Harandi, Vahid

M. Vorob'ev, Mikhail

Ma, Chih-Ming

Ma, Cong

Ma, Junfeng

Ma, Junying

Ma, Liang

Ma, Long

Ma, Qianli

Ma, Qingyong

Ma, Xiaoli

Ma, Xueying

Ma, Yong

Ma, Yufei

Ma, Yuping

Ma, Yurong

Ma, Zhen

Ma, Zhengxin

Maász, Gábor

Macaluso, Roberto

Maccagno, Massimo

Maccallini, Cristina

Macchi, Beatrice

Macdonald, Thomas

MacGillavry, Harold D.

Mach, Mojmir

Machado, Diana

Machado, Fabricio

Machado, Michel Mansur

Machado, Raul

Machetti, Fabrizio

Machrafi, Hatim

Macías, Ramón

Maciej, Strzemski

Maciejewska, Dorota

Maciel, Maria Inês Sucupira

Maciuk, Alexandre

Mack, John

Mackereth, Cameron

MacKinnon, Neil

Maçôas, Ermelinda 
Macone, Alberto

Macreadie, Ian

Mączka, Mirosław

Mączka, Wanda

Mączyński, Marcin

Madala, Hanumantha Rao

Madej, Katarzyna

Madhukar, Burra

Madhyastha, Radha

Madine, Jill

Madka, Venkateshwar

Madonov, Pavel

Madrid, Alejandro

Madsen, Steen J.

Madureira, Patrícia A.

Madurga, Sergio

Mady, Carlos Eduardo Keutenedjian

Mady, Mohamed F.

Madzhidov, Timur

Maeda, Hayato

Maehara, Shoji

Maeng, Han-Joo

Maeno, Zen

Maes, Evelyne

Maestri, Elena

Maestro, Miguel

Maffei, Francesca

Maffei, Gianluca

Maffia, Michele

Mafu, Sibongile

Maga, Giovanni

Magalhães, Geraldo S.

Magalhães, Solange

Magdalena, Woźniczka

MaGee, David

Maggi, Filippo

Magiatis, Prokopios

Magkoev, Tamerlan T.

Magni, Mirko

Magoulas, George

Magrassi, Lorenzo

Magrioti, Victoria

Magrone, Thea

Mague, Joel T.

Magyari, Klara

Mahapatra, Ajit K.

Mahar, Rohit

Mahasenan, Kiran

Mahata, Manoj Kumar

Mahato, Neelima

Mahdavi, Mahboobe
Mahdavian, Elahe

Maher, Pamela

Mahmudov, Kamran T.

Maia, Cláudio

Maicas, Sergi

Maidaniuc, Andreea

Maidannyk, Valentyn

Maier, Camelia

Maietta, Saverio

Maietti, Annalisa

Mainardi, Marco

Maioli, Silvia

Mair, Lamar O.

Maireles Torres, Pedro Jesus

Maisetta, Giuseppantonio

Maixent, Jean Michel

Maj, Malgorzata

Majcher, Malgorzata A.

Majdan, Marek

Majewski, Michał

Majewski, Pawel

Majewski, Sebastian

Majireck, Max

Majkowska-Pilip, Agnieszka

Majolino, Domenico

Majtan, Juraj

Majumder, Avisek

Majumder, Kaustav

Makabe, Hidehumi

Makarevich, Pavel

Makareviciene, Violeta

Makarov, Igor

Makarova, Katerina

Makarska-Bialokoz, Magdalena

Makaryan, Taron

Maker, Garth

Maki, Yasuyuki

Makino, Toshiaki

Makishima, Makoto

Makky, Ali

Makosza, Mieczyslaw

Makowski, Mariusz

Maksimchuk, Nataliya

Maksimov, Anton Lvovich

Maksimowski, Paweł

Makunga, Nokwanda P.

Makvandi, Pooyan

Malaguarnera, Mariano

Malaguarnera, Michele

Malakhov, Dmitri

Malandrino, Graziella 
Malapelle, Umberto

Malawska, Barbara

Maldonado Hodar, Francisco José

Maldonado, José-Luis

Małecka, Magdalena

Malek, Kamilla

Malemud, Charles J.

Malenović, Anđelija

Males, Zeljan

Malfa, Giuseppe Antonio

Malfeito-Ferreira, Manuel

Mali, Gregor

Malik-Gajewska, Magdalena

Malińska, Dominika

Malitesta, Cosimino

Malkin, Alexander

Mallah, Talal

Mallamace, Domenico

Mallat, Ariane

Malliavin, Thérèse

Mallipeddi, Prema Latha

Mallouchos, Athanasios

Mallow, Ole

Malmström, Lars

Malucelli, Giulio

Malvano, Francesca

Malwal, Satish R.

Maly, Pavel

Malzert-Fréon, Aurélie

Mamat, Constantin

Mamba, Bhekie

Mamidi, Narmidi

Mammeri, Fayna

Manabe, Kei

Manabe, Shino

Manabe, Yuki

Managò, Stefano

Manakhov, Anton

Manca, Maria Letizia

Mancianti, Francesca

Mancini, Ines

Manco, Giuseppe

Mancuso, Raffaella

Mandard, Stéphane

Mandati, Vinay

Manders, Peggy

Manderville, Richard A.

Mandoli, Alessandro

Mandrioli, Roberto

Manera, Clementina

Manes, Jordi
Manetti, Dina

Manetti, Mirko

Manfredini, Stefano

Mangani, Stefano

Maniam, Subashani

Manian, Avinash P.

Manicardi, Alex

Manini, Paola

Manivannan, Nadarajah

Mannari, Vijay

Mannello, Ferdinando

Manner, Virginia W.

Mannina, Luisa

Mannino, Giuseppe

Mannino, Saverio

MANNO, Daniela Erminia

Manocha, Gunjan

Manos, Manolis

Manouras, Theodoros

Manoury, Eric

Manousi, Natalia

Mansell, Peter

Mansi, Rosalba

Mansikkamäki, Akseli

Mansuri, Shahid

Mantell, Casimiro

Mantell, Lin

Manthy, John

Mantione, Daniele

Mäntylä, Elina

Mantzioris, Evangeline

Mantzourani, Ioanna

Mantzouridou, Fani

Manyes, Lara

Manzano, Blanca R.

Manzano, Raquel

Manzhos, Sergei

Manzotti, Gloria

Mao, Yuanbing

Mao, Zongwan

Marakos, Panagiotis

Maráková, Katarína

Maramai, Samuele

Marangon, Matteo

Marangoni, Alejandro

Maranzana, Andrea

Marasco, Daniela

Marat-Mendes, Rosa

Maraveas, Chrysanthos

Marbacher, Serge

Marc, Gabriel 
Marcal, Helder

Marceau, François

Marchetti, Alessandra

Marchetti, Fabio

Marchetti, Mario

Marchetti, Marta

Marchetti, Nicola

Marchev, Andrey

Marcinčáková, Dana

Marciniec, Krzysztof

Marcinkowski, Łukasz

Marco, Francisco

Marco-Contelles, Jose Luis

Marcos, Marcos

Marcos, Paula M.

Marcu, Ioan Cezar

Maréchal, Jean-Didier

Marek, Kieliszek

Marengo, Barbara

Marengo, Mauro

Mareș, Mihai

Maresca, Marc

Marfin, Yuriy

Margarucci, Sabrina

Margheritis, Eleonora

Mărghitaş, Liviu A.

Margiolaki, Irene

Marhuenda-Egea, Frutos Carlos

María A., Perillo

Maria Teresa, Frangipane

Maria, Teresa M. R.

Marian, Brigitte

Mariani, Alessandro

Mariani, Stefano

Mariani-Costantini, Renato

Mariappan, Kadarkaraisamy

Marić, Milan

Marichev, Kostiantyn

Marin, Jose J. G.

Marin, Luminita

Marin, Ricardo Mallavia

Marina, María Luisa

Marinescu, Maria

Marini, Elisabetta

Marini, Federico

Marini, Herbert Ryan

Marino, Angela

Marinova, Vera

Marius, Bodor

Mark S., Johnson

Markiewicz, Wojciech T.
Markopoulou, Olga

Markou, Giorgos

Markova, Svetlana

Markova-Car, Elitza P.

Marković, Ksenija

Markovitsi, Dimitra

Markowicz-Piasecka, Magdalena

Markt, Rudolf

Markuszewski, Michel

Marlin, Nathalie

Marminon, Christelle

Marmiroli, Marta

Marocco, Antonello

Marote, Ana

Marpu, Sreekar Babu

Marques Da Silva, Dorinda

Marques, Alexandra P.

Marques, Ana Clara

Marques, M. Matilde

Marquez, Edgar

Marr, Andrew C.

Marra, Alberto

Marra, Fabrizio

Marrazzo, Agostino

Marrazzo, Pasquale

Marrocchi, Assunta

Marrone, Rafaelle

Marrs, James A.

Marrugo-Negrete, José Luis

Marsche, Gunther

Marshall, Paul

Marsillach, Judit

Marsol, Alexis

Marta, Plonska-Brzezinska

Martano, Giuseppe

Martella, Giuseppina

Martelli, Fausto

Martí, Eva

Marti, Guillaume

Martí, Sergio

Martin Lawson, Ata

Martin, Christopher B.

Martin, Francisco

Martin, Franck

Martin, Ian

Martin, James D.

Martin, Jan M. L.

Martín, Margarita

Martín, María Elena

Martin, Olivier

Martin, Rachel 
Martin, Rainer

Martinek, Tamás A.

Martínek, Václav

Martinez Alonso, Marta

Martínez Ávila, Guillermo Cristian

Guadalupe

Martinez De Lizarrondo, Sara

Martinez Hernandez, Elias

Martínez López, José Israel

Martinez Martinez, Virginia

Martínez Rodrigo, Javier

Martínez Urreaga, Joaquín

Martínez, Alberto

Martínez, Ana

Martínez, Constantino

Martínez, Cristina

Martínez, Sidonia

Martinez, Vicente

Martínez-Cifuentes, Maximiliano

Martínez-Espinosa, Rosa María

Martínez-García, Marcos

Martínez-Gómez, Pedro

Martínez-Lillo, José

Martinez-Mayorga, Karina

Martínez-Monteagudo, Sergio I.

Martinez-Moreno, Carlos

Martinez-Navarrete, Gema

Martínez-Palou, Rafael

Martínez-Rodríguez, Sergio

Martínez-Sánchez, Noelia

Martínez-Tomé, María José

Martínez-Tong, Daniel E.

Martinez-Valladares, Maria

Martinez-Villaluenga, Cristina

Martín-Gil, Jesus

Martinho, José Manuel Gaspar

Martini, Daniela

Martini, Petra

Martini, Silvana

Martínková, Ludmila

Martín-Molina, Alberto

Martinotti, Simona

Martín-Ramos, Pablo

Martín-Rapún, Rafael

Martins, Alice

Martins, Ian

Martins, José A.

Martins, Luisa

Martins, M. Rosario

Martins, Mónia A. R.

Martins, Natalia
Martins, Rosário

Martins, Sonia

Martins, Verónica C.

Martins-Bessa, Ana

Marto, Joana

Martos, Perry

Martos, Vanessa

Martra, Gianmario

Martucci, Annalisa

Martucciello, Nadia

Marty, Jean-Daniel

Martz, Francoise

Maruca, Annalisa

Marucci, Gabriella

Maruyama, Ichiro

Maruyama, Rika

Marverti, Gaetano

Marzaro, Giovanni

Marzec, Anna

Marzo, Leyre

Marzocco, Stefania

Marzorati, Stefania

Marzullo, Vincenzo Manuel

Masaki, Takayuki

Masaoka, Tatsuhiro

Mascia, Leno

Masetti, Antonio

Mashima, Ryuichi

Masi, Marco

Maslak, Veselin R.

Masłyk, Maciej

Mason, Nigel

Maspoch, Santiago

Massa, Antonio

Massadi, Omar Al

Massari, Serena

Masselli, Claudia

Massiani, Pascale

Massimiliano, Cordaro

Massiot, Georges

Massotte, Dominique

Massoud, Salah

Mastanjević, Kristina

Masternak, Joanna

Mastinu, Andrea

Mastrogianni, Orthodoxia

Mastrotto, Francesca

Masu, Hyuma

Masuda, Hideki

Masullo, Mariorosario 
Mata, Ricardo

Matanović, Ivana

Matassa, Silvio

Matczak, Piotr

Matczak-Jon, Ewa

Mateescu, Mircea Alexandru

Matei, Ioana

Mateo, Cesar

Mateo, Diego

Mateo, Reyes

Materazzi, Stefano

Materska, Małgorzata

Matesanz, Ana I.

Matesic, Lidia

Mateus, Pedro

Mathe, Ewy

Mathew, Kattathu

Mathieu, Didier

Mathieu, Marchivie

Mathivathanan, Logesh

Matiadis, Dimitris

Matichenkov, Vladimir

Matikonda, Siddharth

Matkowski, Adam

Matos, Inês

Matos, Manuel

Matosiuk, Dariusz

Matouskova, Petra

Mátravölgyi, Béla

Matsidik, Rukiya

Matsoukas, Minos

Matsubara, Kouki

Matsuda, Akio

Matsuda, Tomoko

Matsuda, Yoko

Matsuda, Yu

Matsuda, Yudai

Matsugi, Masato

Matsugo, Seiichi

Matsumoto, Kazuya

Matsumoto, Ken'ichiro

Matsumoto, Shoji

Matsumoto, Takayuki

Matsumoto, Yasuhiko

Matsunaga, Kazuhisa

Matsunami, Katsuyoshi

Matsuo, Hiroshi

Matsuo, Tatsuhito

Matsuo, Yukiko

Matsuura, Bunzo

Matsuura, Kazunori
Matsuya, Yuji

Matta, Cherif

Mattarelli, Maurizio

Matthews, Michael A.

Matthews, Peter

Matthews, Susan

Matthies, Michael

Mattioli, Anna Vittoria

Mattivi, Fulvio

Mattoo, Autar K.

Mattos, Carla

Matuszek, Karolina

Matwijczuk, Arkadiusz

Matyáš, Robert

Matysiak, Joanna

Mauk, Michael G.

Maurer, Andreas

Mauri, Pierluigi

Maurício Alves, Da Motta Sobrinho

Mauriz, Elba

Maurizi, Antonio

Mauro, Matteo

Maury, Olivier

Mautner, Franz

Maveyraud, Laurent

Mavri, Janez

Mavrogonatou, Eleni

Mavromoustakos, Thomas

Mavumengwana, Vuyo

Mawatari, Yasuteru

May, Andrew

Mayence, Annie

Mayer, Dirk

Mayer, Rupert

Mayer, Thomas

Mayer-Gall, Thomas

Mayorga-Martinez, Carmen C.

Mays, Jacqueline W.

Mayumi, Koichi

Mayyas, Mohannad

Mazaira, Gisela

Mazej, Zoran

Mazerbourg, Sabine

Mazin, Alexander

Mazivila, Sarmento

Mazumder, Kishor

Mazur, Andrzej

Mazur-Bialy, Agnieszka

Mazzafera, Paulo

Mazzanti, Michele

Mazzoccanti, Giulia 
Mazzola, Eugene P.

Mazzone, Gloria

Mazzoni, Rita

Mba Blázquez, Miriam

Mbey, Jean Aime

Mbita, Zukile

McCarthy, Pumtiwitt

McCartney, Fiona

McClure, James

McComb, Jen

McCormick, Laura J.

McDonald, Paul

McDougal, Owen M.

McFeeters, Robert

McGlinchey, Michael J.

McGrattan, Kevin B.

McIntyre, Dustin L.

McKay, Ryan T.

Mckee, Vickie

McLemore, Gabrielle Lynn

McMillin, Kenneth W.

McMillin, Matthew

McMorran, David

McNeil, David

McPhail, Kerry L.

McPhillie, Martin

Mcreynolds, Katherine D.

McSweeney, Matthew B.

Md Badrul, Alam

Md, Shadab

Meagher, Robert

Meana Paneda, Ruben

Means, Robert T.

Meca, Giuseppe

Meccariello, Rosaria

Mecinović, Jasmin

Męczyńska-Wielgosz, Sylwia

Medana, Claudio

Medarevic, Djordje

Medeiros, Adriane Bianchi Pedroni

Meder, Roger

Medici, Serenella

Medina Juarez, Luis Angel

Medina, Scott $\mathrm{H}$.

Medina-Franco, José L.

Medina-González, Yaocihuatl

Medina-Ramirez, Adriana

Medio-Simón, Mercedes

Medová, Michaela

Medunic, Gordana

Medved, Igor
Medvidović-Kosanović, Martina

Meegan, Mary J.

Meerson, Ari

Meeta, Gera

Megiel, Elżbieta

Mehandzhiyski, Aleksandar

Mehariya, Sanjeet

Mehl, Florence

Mehta, Sunali

Mei, Yang

Meier-Menches, Samuel M.

Meijide, Francisco

Meiri, David

Meister, Isabel

Mejía-Salazar, Jorge Ricardo

Melcher, Karsten

Mele, Giuseppe

Meléndez-Alafort, Laura

Melendi-Espina, Sonia

Melguizo-Guijarro, Manuel

Melini, Valentina

Melinte, Violeta

Melko, Joshua

Melkonyan, Ferdinand

Mellano, Maria Gabriella

Melman, Artem

Melo, André

Melo, Maria J.

Melo, Pedro

Melo, Priscilla

Melucci, Dora

Memo, Maurizio

Memon, Tosifa

Men, Yongjun

Mena Rejón, Gonzalo Joaquín

Menasché, Philippe

Mendel, Marta

Mendes, Adriano

Mendiola, José

Mendoza-Nuñez, Victor Manuel

Menegatti, Stefano

Meneghello, Roberto

Meneghetti, Fiorella

Menendez, Esther

Menezes, Helvécio Costa

Menezes, Irwin Rose Alencar

Menezes, José C. J. M. D. S.

Menezes, Prashanth

Meng, Dong

Meng, Ge

Meng, Qingshi 
Meng, Xiangchao

Meng, Xiaoli

Menger, Fredric M.

Menghini, Luigi

Menichetti, Stefano

Menke, André

Menkissoglu-Spiroudi, Urania

Menna, Marialuisa

Menon, Nishanth Venugopal

Mensik, Miroslav

Mequanint, Kibret

Merah, Othmane

Mercader, Josep

Mercandelli, Pierluigi

Mercante, Luiza

Mercuri, Nicola Biagio

Mereiter, Stefan

Mergia, Ayalew

Merighi, Adalberto

Mériguet, Guillaume

Merino Peláez, Gracia

Merino, Gabriel

Merino-Montiel, Penelope

Merlino, Antonello

Mernyák, Erzsébet

Merola, Joseph S.

Mertas, Anna

Merten, Christian

Meshram, Chetan

Mesquita, João

Messa, Piergiorgio

Messinger, Robert

Messyasz, Beata

Mester, Patrick-Julian

Mestre, Ana S.

Methling, Karen

Mewis, Ryan E.

Meyer, Andreas

Mezule, Linda

Mezzetta, Andrea

Mi, Fwu-Long

$\mathrm{Mi}$, Lan

Miao, Lingling

Miao, Qingqing

Micale, Nicola

Miceli, Alessandro

Miceli, Natalizia

Michaelakis, Antonios

Michaelian, Karo

Michaelis, Martin

Michail, Christos
Michalak, Agnieszka

Michalik, Jan

Michalska, Anna

Michaud, Guillaume

Michaud, Philippe

Michaud-Soret, Isabelle

Michel, Magdalena M.

Michel, Piotr

Michelacci, Yara M.

Micheletti, Gabriele

Michelle, Palmieri

Michl, Thomas

Michna, Aneta

Micic, Miodrag

Micillo, Raffaella

Micucci, Matteo

Midgley, Adam

Mieczkowski, Adam

Miecznikowski, John

Miekisch, Wolfram

Miękus, Natalia

Mielańczyk, Anna

Mielczarek, Przemyslaw

Mierzejewska, Jolanta

Migaud, Marie E.

Migliori, Carmela Anna

Miguel, Ángel Aguirre

Miguel, Maria Da Graça Costa

Mihai, Marcela

Mihajlović, Ivana

Mihasan, Marius

Mija, Alice

Mika, Delphine

Mikac, Lara M.

Mikata, Yuji

Mikhelson, Konstantin

Miki, Yasuhiro

Mikolajczak, Przemyslaw

Mikros, Emmanuel

Mikstacka, Renata

Mikulic-Petkovsek, Maja

Mikulski, Dawid

Mikuš, Peter

Milán-Carrillo, Jorge

Milanese, Chiara

Milanesio, Marco

Milardi, Danilo

Milasius, Rimvydas

Milazzo, Maria Francesca

Milczarek, Magdalena

Milczarek, Małgorzata 
Mildren, Rich

Milea, Demetrio

Milella, Luigi

Miletić, Goran I.

Miletín, Miroslav

Milham, Paul

Milia, Egle

Milic, Natasa

Milke, Ralf

Milković, Lidija

Milledge, John

Miller, Justin

Miller, Reinhard

Millet, Maurice

Millet, Óscar

Mills, German

Mills, Ken

Milojević-Rakić, Maja

Miloso, Mariarosaria

Milton, Iñaki

Mimica-Dukic, Neda

Min, Kyeong-Sik

Minakshi, Manickam

Minami, Atsushi

Minami, Ichiro

Minami, Masaaki

Minami, Tsuyoshi

Minami, Yasunori

Minbiole, Kevin P. C.

Mincheva, Rosica

Mindt, Thomas

Minehan, Thomas G.

Mineva, Tzonka

Minguez, Laetitia

Miniero, Daniela Valeria

Minkiewicz, Piotr

Minnaar, Phillip

Minnelli, Cristina

Mino, Lorenzo

Minocha, Subhash

Minofar, Babak

Minorics, Renáta

Minutolo, Filippo

Minutti, Carlos

Miranda, Claudio

Miranda, Cristobal

Miranda, Jose M.

Miranda, Katrina

Miriam Maria, De Resende

Mirkhani, Seyyed Alireza

Mirković, Milica
Mironescu, Monica

Mironova, Maria

Miroslaw, Barbara

Mirza, Muhammad Usman

Mirzaeva, Irina

Misaelides, Panagiotis

Miseki, Yugo

Miseta, Attila

Mishima, Masaki

Mishra, Amit Kumar

Mishra, Nagendra N.

Mishra, Nigam

Mishra, Pawan Kumar

Mishra, Rosalin

Mishra, Yogendra

Mishyna, Maryia

Misicka, Aleksandra

Misra, Rajesh Chandra

Mistrik, Martin

Misul, Daniela

Mita, Giovanni

Mitaine-Offer, Anne-Claire

Mitakou, Sofia

Mitani, Shohei

Mitani, Takakazu

Mitchell, Angela M.

Mitchell, Cassie S.

Mitchell, Scott G.

Mitchell, Todd

Mitjans Arnal, Montserrat

Mitoraj, Mariusz

Mitran, Gheorghiţa

Mitran, Raul-Augustin

Mitsou, Evdokia K.

Mitsudo, Koichi

Mitulovic, Goran

Miyagawa, Hayato

Miyamae, Yusaku

Miyamoto, Koji

Miyanaga, Akimasa

Miyata, Yoshiki

Miyoshi, Daisuke

Miyoshi, Norio

Mizerska, Urszula

Mizerska-Kowalska, Magdalena

Mizobata, Tomohiro

Mizoshiri, Mizue

Mizumoto, Shuji

Mizuno, Cassia S

Mladěnka, Přemysl

Mladenović, Milan 
Mlcek, Jiri

Mleczek, Miroslaw

Mlejnek, Petr

Mlostoń, Grzegorz

Mnif, Wissem

Moad, Graeme

Mobbili, Giovanna

Moccia, Stefania

Mochizuki, Hitoshi

Mochizuki, Shinichi

Močibob, Marko

Mock, Beverly

Modec, Barbara

Modenutti, Carlos

Modica, Maria

Modolo, Luzia Valentina

Modugno, Francesca

Modukuri, Ramkumar

Moein, Mohammad Mahdi

Moghtadernejad, Sara

Mogielnicki, Andrzej

Mohamed, Azza H.

Mohamed, Sharmarke

Mohamed, Tamer

Mohammed, Altaf

Mohammed, Sohaib

Mohankumar, Kumaravel

Mohanty, Joy G.

Mohles, Volker

Moita, Ana Sofia

Mojzych, Mariusz

Mok, Daniel Kam-Wah

Mokshin, Anatolii V.

Moldogazieva, Nurbubu T.

Moldovan, Mirela

Moldovan, Radu-Cristian

Moldoveanu, Serban C.

Molina Salinas, Gloria María

Molina-Cerrillo, Javier

Molina-Espeja, Patricia

Molina-Santiago, Carlos

Molinie, Roland

Moll, Henri C.

Mollace, Vincenzo

Mollica, Adriano

Mollica, Giulia

Molognoni, Luciano

Mołoń, Mateusz

Moloney, Mark G.

Molteni, Giorgio

Momekov, Gregori
Momekova, Denitsa B.

Monai, Matteo

Monari, Antonio

Moncalián, Gabriel

Mondal, Kunal

Mondal, Mukulesh

Mondanelli, Giada

Monge, David

Moni, Lisa

Monica-Maria, Baia

Monika, Waksmundzka-Hajnos

Moniuszko-Szajwaj, Barbara

Moniz, Tânia

Monnerat, Gustavo

Monroe, Mary Beth Browning

Monsalve, Maria

Montagne, Kevin

Montagnon, Tamsyn

Montalvo González, Efigenia

Montalvo-Rodríguez, Rafael

Montanez, Julio Cesar

Montaño, Gabriel A.

Montarolo, Francesca

Montecchio, Daniele

Montecucco, Fabrizio

Monteiro, Carlos

Monteiro-Neto, Valério

Montenarh, Mathias

Montenegro, Iván

Montero, Olimpio

Montes Morán, Miguel A.

Montesano, Camilla

Montesano, Domenico

Montesarchio, Daniela

Montes-García, Verónica

Monti, Daniela

Monti, Marco

Montiel-Smith, Sara

Montoliu-Gaya, Laia

Montone, Carmela Maria

Montoro, Paola

Montouillout, Valérie

Montoya-Castillo, Andrés

Monts, David

Moon, Geon Dae

Moon, Il Soo

Moon, Jong-Seok

Moon, Minho

Moon, Yuseok

Moore, Stuart

Moore, Tara 
Moosmann, Bernd

Mor, Amram

Morabito, Rossana

Moraca, Federica

Moraga, Javier

Morais, Mauricio

Morais, Tânia S.

Morak-Młodawska, Beata

Morales Martín, Ana

Morales Suárez-Varela, María M.

Morales, Javier

Morales, Jose Manuel

Morales, Patricia

Morales, Paula

Morales-de La Peña, Mariana

Morales-González, José Antonio

Morales-Morales, David

Moran, Wesley

Moratti, Stephen C

Moravcová, Dana

Morbidelli, Lucia

Morcillo, Miguel Ángel

Mordalski, Stefan

Moreira Gonçalves, Luís

Moreira, Daniele

Moreira, Ibério De P. R.

Moreira, Irina S.

Moreira, Vânia M.

Morel, Bertrand

Morel, Mauricio

Moreno, Abel

Moreno, Adrian

Moreno, Antonio D.

Moreno, Diego A.

Moreno, Esther

Moreno, Jordi

Moreno, Juan J.

Moreno, Maria João

Moreno, Miguel

Moreno-Piraján, Juan Carlos

Moreno-Rojas, José Manuel

Moretto, Alessandro

Morgan, David

Morgan, Kevin

Morganti, Pierfrancesco

Morgunov, Igor

Mori, Mattia

Mori, Seiji

Mori, Shigeki

Mori, Wasuke

Morianos, Ioannis
Morigi, Rita

Morikawa, Toshio

Morillas, Leandro

Morimoto, Yuma

Morimura, Shigeru

Morini, Luca

Morisseau, Christophe

Morita, Naoki

Morita, Nobuyoshi

Moriuchi-Kawakami, Takayo

Moriwaki, Hiroshi

Moriyama, Katsuhiko

Morjani, Hamid

Morkunas, Iwona

Mormann, Michael

Mornar, Ana

Morocho, Vladimir

Morozov, Vitaly A.

Morrison, Juliet

Morrone, Aldo

Morselli, Davide

Mortara, Lorenzo

Mortier, Jérémie

Morzelle, Maressa Caldeira

Morzycki, Jacek W.

Mosca, Luciana

Moschini, Roberta

Moschopoulou, Ekaterini

Moschopoulou, Georgia

Mosharov, Eugene V.

Moskaleva, Lyudmila

Mosnáček, Jaroslav

Mosqueira, Diogo

Mosqueira, Matias

Mosquera, Marta

Mossine, Andrew V.

Mossine, Valeri V.

Mostafavi, Mehran

Mot, Augustin

Mota, Antonio J.

Mota-Morales, Josué D.

Motoyama, Keiichi

Motrescu, Iuliana

Mou, Yongchao

Mouahid, Adil

Mouga, Teresa

Moujir, Laila M.

Moura, Nuno M. M.

Moura, Rute

Mourão, Paulo A. S.

Mourtzinos, Ioannis 
Mousley, Carl

Moussaoui, Younes

Moustakas, Michael

Moyano, Albert

Moyano-Mendez, Jose Ramon

Moya-Ramírez, Ignacio

Moyseowicz, Adam

Mozaffari, Saeed

Mozejko-Ciesielska, Justyna

Mozgawa, Włodzimierz

Mozhzhukhina, Nataliia

Mozo, Juan Daniel

Mozumder, Md. Salatul Islam

Mphahlele, Malose Jack

Mravec, Jozef

Mráz, Jaroslav

Mrówczyński, Radosław

Mrowiec-Białoń, Julita

Mróz, Zenon

Mshvildadze, Vakhtang

$\mathrm{Mu}$, Qingxin

Muccilli, Vera

Muceniece, Ruta

Mucha, Igor

Muchowicz, Angelika

Muchowska, Kamila B.

Muders, Michael

Mueller, Paul A.

Mueller, Thomas J.J.

Mughal, Amreen

Muhle-Goll, Claudia

Mühlenhoff, Ulrich

Mujtaba, Muhammad

Mukai, Hidehito

Mukai, Rie

Mukhametzyanov, Timur A.

Mukherjee, Shayanti

Mulak, Agata

Mulas, Maurizio

Mulcahy, Ben

Mulherkar, Shalaka

Mullen, Jack

Müller, Carsten

Muller, Christian

Müller, Christoph

Muller, Eric

Müller, Gerhard

Müller, Joachim

Müller, Juliane

Müller, Kajetan

Müller, Karsten
Muller, Patricia A. J.

Müller, Thomas

Müller, Thomas Ernst

Müller, Uwe

Müller, Werner E. G.

Müller-Vahl, Kirsten R.

Mulmudi, Hemant Kumar

Munekata, Paulo E.S.

Muñoz, Asunción

Muñoz, Eduardo

Muñoz, Marcelo Andrés

Muñoz-Castro, Alvaro

Muñoz-Mingarro, Dolores

Muntean, Danina

Muntean, Delia

Munteanu, Florentina-Daniela

Muntimadugu, Eameema

Munusamy, Elango

Mura, Umberto

Murab, Sumit

Murakami, Kazuma

Murakami, Yasufumi

Muramatsu, Atsushi

Muranaka, Atsuya

Murase, Daiki

Murata, Toshihiro

Muratani, Masafumi

Muratova, Anna

Muravyev, Nikita V.

Murdan, Sudax

Muresan, Liana

Muresan, Vlad

Murias, Marek

Murillo, Walter

Murkovic, Michael

Murphy, Caroline S.

Murray, Jane S.

Murray, Keith

Murtinho, Dina

Murugan, N. Arul

Muscara, Marcelo N.

Musco, Giovanna

Musgrave, Ian

Musiani, Francesco

Musilek, Kamil

Musioł, Robert

Musk, Bill

Musso, Ioana

Must, Indrek

Musumeci, Chiara

Musumeci, Domenica 
Mus-Veteau, Isabelle

Muszyńska, Bożena

Muthukumar, Kannan

Muthuramu, Ilayaraja

Mutiga, Samuel K.

Mutlu, Ayse Sena

Muto, Kei

Muxel, Sandra Marcia

Muzafarov, Aziz

Muziol, Tadeusz

Muziol, Tadeusz Mikolaj

Mwanza, Mulunda

Myers, Timothy G.

Mykhaylyk, Oleksandr O.

Myllymäki, Sami

Myong Jae, Yoo

Myongwon Lee, Lucia

Mysliveček, Jaromír

Mysliwiec, Tami

$\mathrm{Na}$, Dokyun

$\mathrm{Na}$, Kun

Nabae, Yuta

Nadăş, George Cosmin

Nadiminty, Nagalakshmi

Nagaoka, Shoji

Nagarajan, Balaji

Nagata, Naoto

Nagatoishi, Satoru

Nagatsu, Akito

Nagatsugi, Fumi

Nagel, Raimund

Nagel, Stefan

Nagulapalli Venkata, Kalyan

Nagy, Corina

Nagy, Csaba Levente

Nagy, Endre

Nagy, László

Nagy, Miklós

Nagy, Tamás

Nagy, Veronika

Nahm, Kee Suk

Nahra, Fady

Naini, Santhosh Reddy

Nair, Anroop B.

Nair, Sandeep Sudhakaran

Nair, Sreenath

Naito, Toshio

Najda, Agnieszka

Najeeb, Shariq

Najlah, Mohammad

Najmanová, Iveta
Nakabayashi, Koji

Nakabayashi, Seiichiro

Nakabayashi, Takakazu

Nakagaito, Antonio Norio

Nakagawa, Atsushi

Nakagawa, Hiroshi

Nakagawa, Tetsuya

Nakagawa, Yoshinao

Nakagawa-Goto, Kyoko

Nakamaru-Ogiso, Eiko

Nakamura, Issei

Nakamura, Mitsutaka

Nakamura, Soichiro

Nakano, Hiroto

Nakano, Shogo

Nakano, Shu-ichi

Nakano, Tamaki

Nakashima, Kenichi

Nakashima, Souichi

Nakata, Norio

Nakata, Satoshi

Nakayama, Masayoshi

Nakayama, Mizuho

Nakayama, Yuushou

Nakazato, Gerson

Nakhjavani, Maryam

Nalewajko-Sieliwoniuk, Edyta

Nam, Il-Woo

Nam, Inho

Nam, Ki Hyun

Nam, Tae Gyu

Namasivayam, Vigneshwaran

Namgaladze, Dmitry

Namihira, Masakazu

Nanda, Satyabrata

Nanduri, Ravikanth

Nandy, Arpita

Nanno, Masanobu

Nano, Adela

Nanoff, Christian

Nantasenamat, Chanin

Napoli, Anna

Napoli, Edoardo Marco

Napolitano, Alessandra

Narayan, Mahesh

Nardecchia, Stefania

Nardi, Monica

Nardi, Serenella

Nardin, Tiziana

Narita, Shin-ichiro

Narkar, Ameya 
Naryzhny, Stanislav

Nasarre, Patrick

Nascimento, Luís Adriano Santos Do

Nascimento, Yuri

Nash, Robert

Nassar, Zeyad D.

Nastasa, Cristina

Nasti, Rita

Nastuta, Andrei Vasile

Natale, Paolo

Natali Sora, Isabella

Natali, Francesca

Natarajan, Arutselvan

Nath, Arijit

Nativi, Cristina

Naumova, Anna V.

Naumovski, Nenad

Naumowicz, Monika

Naushad, M

Navarra, Michele

Navarrete-Vazquez, Gabriel

Navarro, Elena

Navarro, Elisa

Navarro, Rodrigo

Navarro-Hoyos, Mirtha

Navickiene, Sandro

Naviglio, Daniele

Nawaz, Muhammad

Nawirska-Olszańska, Agnieszka

Nawrot, Barbara

Nawrot-Hadzik, Izabela

Nayak, Tapan

Nayak, Usha Y.

Nazarchuk, Evgeny

Nazarkina, Zhanna

Nazarski, Ryszard B.

Nazemi, Ali

Nazir, Rashid

Naznin, Most Tahera

Nazzaro, Filomena

Ndika, Joseph

Nebot, Carolina

Nebra, Noel

Neculita, Carmen Mihaela

Neda, Ion

Nedialkov, Paraskev T.

Nees, Matthias

Negahdar, Leila

Neginskaya, Maria A.

Negrea, Adina

Negrea, Petru
Negri, Giuseppina

Negroni, Luc

Nehela, Yasser

Neilson, Andrew

Neira, José L.

Neiva, Duarte M.

Nelson, Andrew

Nelson, Randy

Nelson, Richard W.

Nelyubina, Yulia

Nemec, Ivan

Németh, Róbert

Nemoto, Kiyomitsu

Nemykin, Victor

Nenadis, Niknen

Nenajdenko, Valentine G.

Neng, Nuno

Neo, Ana G.

Neogi, Ujjwal

Neophytou, Christiana M.

Nesmelova, Irina

Nesterov, Dmytro

Nesterova, Oksana V.

Nestler, Ulf

Neubauer, Heidi

Neubert, Reinhard

Neugebauer, Dorota

Neugebauer, Witold

Neunert, Grazyna

Neureiter, Daniel

Neuwald, Daniel Alexandre

Nevares, Ignacio

Nevárez-Moorillón, Guadalupe Virginia

Neves, Bruno

Neves, João

Neves, Maria Da Graça P. M. S.

Neves, Vera

Newberg, John T.

Neyerlin, Kenneth Charles

$\mathrm{Ng}$, Andy

$\mathrm{Ng}$, Caroline

$\mathrm{Ng}$, Ho Leung

$\mathrm{Ng}$, Tzi Bun

Ngai, To

Nge, Thi Thi

Ngezahayo, Anaclet

Nguyen, Khoi Tan

Nguyen, Minh Tho

Nguyen, Ngoc A.

Nguyen, Nhat Truong

Nguyen, Thanh Binh 
Nguyen, Trieu

Nguyen, Van Khanh

Nguyen, William

$\mathrm{Ni}$, Bukuo

$\mathrm{Ni}$, Junjun

Niaura, Gediminas

Nicasio, Maria Carmen

Nicholas, Darrel

Nichols, Nancy N.

Niclos Gutiérrez, Juan

Nicolas, Cyril

Nicolau, Elodie

Nicoletta, Fiore Pasquale

Nicoletti, Ferdinando

Nicoletti, Rosario

Nicoletti, Vincenzo

Nicolotti, Orazio

Niculau, Edenilson Dos Santos

Niculescu, Loredan

Nie, Chuanxiong

Nie, Guo-Xing

Nie, Liming

Niedziela, Tomasz

Niedzielski, Przemysław

Niedźwiedzka-Rystwej, Paulina

Nielsen, Peter Eigil

Nielsen, Vance G.

Niemczak, Michal

Niemczyk, Agata

Nieminen, Pentti

Niemirowicz-Laskowska, Katarzyna

Nieoczym, Dorota

Nieto, Gema

Nieto, Manuel

Nieto, Marcelo J.

Nievergelt, Adrian Pascal

Nikitin, Kirill

Nikolaev, Eugene

Nikolaev, Viacheslav

Nikolaevskii, Stanislav A.

Nikolaidis, Nikolaos

Nikolaivits, Efstratios

Nikolakaki, Eleni

Nikolakakis, Ioannis

Nikolakopoulou, Angeliki M.

Nikolay V., Sidorov

Nikolić, Dragan

Nikoloudakis, Nikolaos

Nikolova, Rositca

Nima, Zeid A.

Ninfali, Paolino
Ning, Xuhui

Ninomiya, Satoshi

Nishi, Kosuke

Nishi, Mayumi

Nishida, Ritzuo

Nishihara, Yasushi

Nishikimi, Akihiko

Nishimoto, Yoshio

Nishinaga, Tohru

Nishiwaki, Hisashi

Nishiwaki, Nagatoshi

Nishiyama, Yusuke

Nissen, Lorenzo

Nissinen, Maija

Nisticò, Roberto

Nistor, Ileana Denisa

Nita, Loredana E.

Nitiss, John

Nitulescu, George Mihai

Nitulescu, Mihai

Nitz, Mark

Nitzsche, Bianca

Niu, Ran

Niu, Tianhua

Nixon, Gemma

Nizetic, Sandro

Njar, Vincent C. O.

Nkukwana, Thobela

Noble, Benjamin

Nobre, Luís

Nocentini, Sara

Noda, Takahiro

Noei, Heshmat

Noël, Sébastien

Nogales-Bueno, Julio

Nogami, Makoto

Nogara, Pablo

Nogawa, Toshihiko

Noge, Koji

Noguchi, Hiroshi

Nogueira Perez, Juan Jose

Noguera Artiaga, Luis

Noguera, Rosa

Nogues, Isabel

Nomikos, Tzortzis

Nomngongo, Philiswa Nosizo

Nomura, Mikihiro

Nomura, Seitaro

Nora-Krukle, Zaiga

Norman, Trevor

Norton, Michael 
Nose, Takeru

Noseda, Miguel D.

Nothias, Louis-Félix

Noureddine, Achraf

Novak Kujundžić, Renata

Novak, Pavel

Novič, Marjana

Novikov, Alexander S.

Novinec, Marko

Novio, Fernando

Novío, Silvia

Novotna, Vladimira

Nový, Pavel

Nowacka-Jechalke, Natalia

Nowaczyk, Jacek

Nowak, Adriana

Nowak, Agnieszka

Nowak, Krzysztof

Nowak, Pawel

Nowakowska, Justyna

Nowicka, Grażyna

Nowicki, Janusz

Nozaki, Masami

Nsanzabana, Christian

Ntaikou, Ioanna

Ntalii, Nikoletta

Ntalli, Nikoletta G.

Ntie-Kang, Fidele

Numez, Estrella

Nunes, Alexandra

Nunes, Fernando

Nunes, Leonel

Nuñez, María B.

Nunez, Oscar

Nuñez, Rosario

Nunzio, Vicario

Nurieva, Evgeniya

Nussaume, Laurent

Nuță, Diana Camelia

Nuttall, Patricia A.

Nwaiwu, Ogueri

Nycz, Jacek

Nycz, Jacek E.

Nyman, Tuula Anneli

O’Duill, Miriam L.

O'Hare, Tim

Oancea, Florin

Obata, Tohru

Obata, Yasuko

Obaya, Alvaro J.

Oberley, Rebecca
Obeso, Ana

Oborník, Miroslav

Obrador, Elena

Obreza, Aleš

Obuobi, Sybil Akua Okyerewa

Ocampo-García, Blanca Elí

Ochędzan-Siodłak, Wioletta

Ochi, Shinichiro

Ochiai, Bungo

Ochoa-Reparaz, Javier

Ochowiak, Marek

Oćwieja, Magdalena

Oczkowicz, Maria

Oda, Takuji

Oda, Yukari

Odagi, Minami

Odell, Luke R.

Ogale, Amod A.

Ogawa, Kazuma

Ogawa, Mitsutaka

Oghumu, Steve

Ognyanov, Manol

Ogórek, Rafał

Ogra, Yasumitsu

Ogungbe, Ifedayo

Oh, Jae-Min

Oh, Kyungsoo

Oh, Sangphil

Oh, Seung-Won

Ohgane, Kenji

Ohisa, Satoru

Ohmae, Eiji

Ohms, Gisela

Ohnishi, Kohta

Ohnishi, Shiho

Ohno, Osamu

Ohta, Kiminori

Ohta, Nobuo

Ohta, Shinji

Ojeda-May, Pedro

Ojha, Deepak

Ojima-Kato, Teruyo

Ojo, Peter

Ojstršek, Alenka

Ojwang, Dickson

Okada, Yoshiharu

Okamoto, Masami

Okamoto, Takayuki

Okawara, Toru

Okazawa, Atsushi

Oklinski, Michal Krystian Egelund 
Ok-Seon, Kwon

Okuda, Masayuki

Okumura, Masaki

Okunade, Adewole L.

Okuniewski, Andrzej

Oláh, Attila

Oláh, Julianna

Oláh, Viktor

Olar, Rodica

Olaru, Mihaela

Olaru, Octavian Tudorel

Olech, Marta

Olejar, Kenneth J.

Olejnik, Anna

Olennikov, Daniil

Oleszek, Wieslaw

Olewnik-Kruszkowska, Ewa

Olga, Papadopoulou

Oliani, Sonia Maria

Oliva, Alexis

Oliva, José

Oliva, Romina

Oliveira, Alcineia C.

Oliveira, Ana

Oliveira, Andreia

Oliveira, Guedmiller S.

Oliveira, Hugo M.

Oliveira, Joana

Oliveira, José M.

Oliveira, M. Conceição

Oliveira, Maria Conceição

Oliveira, Paula A.

Oliveira, Rui S.

Oliver, William Thomas

Oliveri, Paolo

Olivero-Verbel, Jesus

Oliviero, Francesca

Oliviero, Giorgia

Olivos-Oré, Luis Alcides

Oliwa, Rafał

Ollero, Mario

Ollevier, Thierry

Olmo, Luis Rojo Del

Olmo-García, Lucía

Olofinjana, Ayodele

Olorunniji, Femi J.

Olson, David E.

Olson, Thomas L.

Olubiyi, Olujide O.

Olyaee, Saeed

Omar, Ahmad A.
Omar, Syed Haris

Omichi, Masaaki

Omidian, Kosar

Omura, Junichi

Ončák, Milan

Onchoke, Kefa

Onduka, Toshimitsu

Ong, Eng Shi

Ong, Hwai Chyuan

Oniga, Ilioara

Oniga, Smaranda Dafina

Oniszczuk, Anna

Oniszczuk, Tomasz

Onnis, Valentina

Ono, Kenji

Onyeagucha, Benjamin Chidi

Onyenwoke, Rob Uche

Onys'ko, Petro

Oostenbrink, Chris

Opaliński, Łukasz

Opatz, Till

Opekar, František

Opitz, Jörg

Oppedisano, Francesca

Oprea, Corneliu Ioan

Oprea, Ovidiu

Oprică, Lăcrămioara

Opsenica, Igor

Oracz, Joanna

Orbach, Ron

Orczyk-Pawiłowicz, Magdalena

Ordaz-Ortiz, José Juan

Ordóñez García, Salvador

Ordoudi, Stella

Orellana-Palma, Patricio

Oreshonkov, Aleksandr

Orfila, Caroline

Orian, Laura

Orinska, Zane

Orkusz, Agnieszka

Orlando, Giustino

Orlić, Sandi

Orlova, Anna

Orłowski, Marek

Ormeci, Alim

Orobtchouk, Regis

Orodnez, Mario

Oroian, Mircea Adrian

Orooji, Yasin

Orosa, José A.

Orozco, Jahir 
Orso, Evelyn

Ortega Castro, Joaquín

Ortega, Daniel

Ortega, María Jesús

Ortega, Natividad

Ortega, Paula

Ortega-Alfaro, María Carmen

Orth, James D.

Ortigueira, Joana

Ortiz Martínez, Víctor Manuel

Orts, Julien

Orynycz, Olga

Orzelska-Górka, Jolanta

Osakada, Kohtaro

Osakada, Yasuko

Osaki, Tomohiro

Osborn, Helen

Osborn, Helen M.i.

Osés, Sandra

Oshima, Yusuke

Osma, Johann

Osman Ahmed, Ahmed

Ośmiałowski, Borys

Osório, Wislei R

Ospina-Millán, Claudia A.

Osses, Nelson

Ostacolo, Carmine

Ostadhassan, Mehdi

Ostoa-Saloma, Pedro

Ostojic, Sergej

Ostrauskaite, Jolita

Ostrowska, Kinga

Ostrowski, Maciej

Otero, José A.

Otero, Marta

Otero-Espinar, Francisco Javier

Othman, Eman M.

Otmačić Ćurković, Helena

Otomo, Takanobu

Otřísal, Pavel

Otsuka, Masami

Otsuka, Yuzuru

Otte, Andrew D.

Ottria, Roberta

Ouellet-Plamondon, Claudiane

Ouguerram, Khadija

Ouvrard, Aimeric

Ouyang, Liliang

Ovando Martínez, Maribel

Oven, Primoz

Overgaard, Jacob
Oves-Costales, Daniel

Ovidi, Elisa

Ovissipour, Reza

Owczarz, Piotr

Oyama, Dai

Oyedeji, Adebola

Ozarowski, Marcin

Ozawa, Koichiro

Ozawa, Shogo

Ozawa, Yoshiki

P. Busardó, Francesco

Pacelli, Settimio

Pacetti, Deborah

Pachauri, Vivek

Pacheco, Neith

Pacheco-Catalán, Daniella

Pacheco-Fernández, Idaira

Pacheco-Londoño, Leonardo C.

Paci, Maurizio

Pacia, Marta Z.

Pacifici, Roberta

Pacifico, Severina

Paciorek-Sadowska, Joanna

Pacios, Luis F.

Paczesny, Jan

Paczkowski, Jon

Padilla-Martínez, Itzia I.

Padilla-Zakour, Olga

Padmanabhan, Venkat

Padnya, Pavel

Padrão, Jorge

Padro, Juan M.

Paduch, Roman

Padula, Matthew P.

Paeng, Kijung

Paepegaey, Anne-Cécile

Pagacz, Joanna

Pagani, Stefania

Paganin, Valdecir Antonio

Pagano, Alessandra

Pagano, Bruno

Pagano, Cinzia

Pagliara, Stefano

Pagotto, Sara

Paik, Younkee

Pailleret, Alain

Paim, Leonardo

Paixão Cansado, Isabel Pestana

Paixao Coelho, Jose Augusto

Paizs, Csaba

Pajak, Beata 
Pajchel, Łukasz

Pajeva, Ilza

Pak, Chanho

Pakdel, Farzad

Pakhomova, Svetlana

Pal, Animesh

Pal, Rumpa

Palacio, Edwin

Palacios, Javier

Palacios-Santander, José María

Paladino, Antonella

Palage, Mariana

Palamà, Ilaria

Palanti, Sabrina

Palavai, Sripal Reddy

Palchoudhury, Soubantika

Palcut, Marián

Palenzuela López, José Antonio

Palermo, Amelia

Palese, Luigi Leonardo

Palinko, Istvan

Pall, Emoke

Pallàs Lliberia, Mercè

Palma, Eleonora

Palma, Miguel

Palmer, Alan

Palmieri, Alessandro

Palmieri, Erika

Palmieri, Valentina

Palomba, Letizia

Palomba, Roberto

Palombi, Laura

Paltinean, Ramona

Palumbo, Giuseppe Alberto

Palumbo, Oriele

Paluszczak, Jarosław

Palyi, Gyula

Palyulin, Vladimir

Pan, Jing

Pan, Jinhe

Pan, Po-shen

Pan, Quanwen

Pan, Shanlin

Pan, Yuan Jiang

Panagiotidis, Mihalis I.

Panaro, Maria Antonietta

Panayiotou, Costas

Panchal, Satyam

Panchapakesan, Balaji

Pandele, Madalina

Panderi, Irene
Pandey, Santosh

Pandit, Santosh

Pandíțh, Anúp

Pandya, Pankita H.

Panek, Jaroslaw

Panek, Jarosłąw

Paneth, Agata

Paniagua, Candelas

Panić, Manuela

Panigati, Monica

Pannerselvam, Suresh

Pannkuk, Evan

Pansare, Sunil V.

Pantazis, Dimitrios A.

Pantić, Milica

Panzella, Lucia

Pao, Chun-Wei

Paola, D'Arrigo

Paolella, Andrea

Paolesse, Roberto

Paoli, Paolo

Paolillo, Mayra

Paolino, Marco

Paolmino, Olga

Paone, Alessio

Pap, József

Papaccio, Gianpaolo

Papachristodoulou, Alexandros

Papadakis, Raffaello

Papadimitriou, Vassiliki

Papadopoulos, Antonios N.

Papadopoulos, Christodoulos E.

Papadopoulos, Dimitrios

Papageorgiou, Anastassios C.

Papagiannitsis, Costas C.

Papagiannopoulou, Dionysia

Papaioannou, Dionissios

Papakyriakou, Athanasios

Papamichael, Emmanuel M.

Papanastasiou, Ioannis

Papathanasiou, Fokion

Papatriantafyllopoulou, Constantina

Papetti, Adele

Papi, Alessio

Papież, Monika

Paplinska, Magdalena

Papoti, Vassiliki T.

Pappa, Aglaia

Pappalardo, Daniela

Pappas, Christos

Paquete, Catarina M. 
Parada, Fabián

Paradela, Alberto

Paradowska-Gorycka, Agnieszka

Paramythiotis, Spiros

Pardío-Sedas, Violeta T.

Pardo, Emilio

Paredes, Adrian

Paredes, Alejandro Javier

Paredez, Alexander

Parenti, Carmela

Parfenova, Helena

Parida, Asish K.

Parida, Sheetal

Parikesit, Arli Aditya

Parisi, Filippo

Parizad, Parisa Abbasi

Park, Byung-Wook

Park, Chul-ho

Park, Chulhun

Park, Daeho

Park, Daesung

Park, Deog-Su

Park, Dong Hyuk

Park, Dong Sun

Park, Han-A

Park, Hee-Soo

Park, Hyun-Woo

Park, Il-Kwon

Park, Inkyu

Park, Jae-Hyung

Park, Jeewoong

Park, Jeong-Hun

Park, Jeyoung

Park, Ji-Won

Park, Jongseung

Park, Joon Sik

Park, Ki Tae

Park, Kyoung-Chan

Park, Kyung-Mok

Park, Min

Park, Mira

Park, Myung Hwan

Park, Sang Ki

Park, Sangmoon

Park, Sangwon

Park, Solmoi

Park, Soo Jean

Park, Sungsu

Park, Wansu

Park, Wooram

Park, Yaewon
Park, Yoo Min

Park, Yoonsu

Park, Young-Seo

Park, Young-Tae

Parker, Linda A.

Parlog, Raluca

Parmeggiani, Fabio

Parpinello, Giuseppina

Parra, Margarita

Parra-Saldívar, Roberto

Parrella, Edoardo

Parrino, Barbara

Parrino, Francesco

Parrotta, Elvira

Parrotta, Luigi

Parsaeimehr, Ali

Parsons, Jason

Pârvu, Alina Elena

Pârvu, Marcel

Pasa, Andre

Pasán, Jorge

Pasbakhsh, Pooria

Pascali, Giancarlo

Pasche, Boris

Paseiro-Cerrato, Rafael

Pasetto, Marco

Pasini, Dario

Paskaleva, Albena

Pasquinelli, Gianandrea

Passamonti, Sabina

Passarella, Daniele

Pasternak, Taras

Pašti, Igor A.

Pastor, Victoria

Pastore, Carlo

Pastore, Roberto

Pastuch-Gawolek, Gabriela

Paszkiewicz, Monika

Paszkiewicz, Sandra

Patankar, Jay V.

Patarata, Luís

Pate, F. Donald

Pateiro, Mirian

Patel, Alok

Patel, Ankit B.

Patel, Bhargav

Patel, Nilkanth

Patenge, Nadja

Pateraki, Irini

Paterson, Brett

Pathak, Pawan 
Patience, Gregory

Patra, Jayanta Kumar

Patriarchi, Tommaso

Patruno, Antonia

Pattammattel, Ajith

Pattison, Graham

Pauer, Werner

Paul, Ananya

Paul, Arghya

Paul, Sanjoy

Paul, Titan

Pauleta, Sofia

Pauliukaite, Rasa

Paulo, António

Paulson, Joel

Paulus, Beate

Pau-Roblot, Corinne

Pautz, Andrea

Pavagadhi, Shruti

Pavanello, Chiara

Pavao, Mauro Sergio

Pavel, Octavian Dumitru

Pavela, Roman

Pavelic, Sandra K.

Paventi, Gianluca

Pavic, Valentina

Pavlić, Branimir

Pavlos, Klepetsanis

Pavlov, Georges M.

Pavlov, Youri I

Pavlovic, Radmila

Pawar, Shrikant D.

Pawar, Snehalata

Pawlak, Andrzej

Pawlicki, Miłosz

Pawluć, Piotr

Pawyta, Miroslawa

Payan-Carreira, Rita

Paz, Cristian

Pazdzior, Katarzyna

Paz-Ferreiro, Jorge

Pazourek, Jiří

Peana, Massimiliano F.

Pecina, Petr

Pecinová, Alena

Peczuh, Mark

Pedatella, Silvana

Pedersen, Henrik

Pedraza Chaverri, José

Pedretti, Alessandro

Peelman, Frank
Peeters, Marloes

Peethambaran, Bela

Pei, Yonggang

Pei, Zhiqiang

Pei, Zingway

Peiretti, Pier Giorgio

Peixe, Tiago Severo

Peixoto, Maria João

Peiyan, Shen

Pék, Zoltán

Pekar, Miloslav

Peleteiro, Mercedes

Pelidou, Sygkliti-Henrietta

Pelka, Robert

Pellegrin, Yann

Pellegrini, Carolina

Pellegrini, Marika

Pellegrino, Roberto Maria

Pellegrino, Sara

Pellei, Maura

Pellico, Juan

Pelosi, Giorgio

Peluffo, Hugo

Peluso, Ilaria

Peluso, Paola

Peña Fernández, María Ángeles

Peña-Amaro, José

Peñalver, Rosa

Peña-Rodríguez, Luis Manuel

Penas, Sonia Eiras

Penesova, Adela

Peng, Bingyin

Peng, Robert $\mathrm{Y}$.

Peng, Shu-Fen

Peng, Wen-Huang

Peng, Zhili

Penn, Raymond

Penna, Claudia

Pentzold, Stefan

Pepi, Milva

Peptu, Cristian

Peral, Angel

Perale, Giuseppe

Perálvarez-Marín, Alex

Perbellini, Luigi

Perche, Fédérico

Percino, Judith

Percival, Susan S.

Perdih, Andrej

Perdih, Franc

Perdomo, Jose 
Perego, Carla

Pereira, Carla

Pereira, David

Pereira, Eliana

Pereira, Filipe

Pereira, Florbela

Pereira, Giuliano Elias

Pereira, Jorge

Pereira, José Alberto

Pereira, José Augusto

Pereira, Leonel

Pereira, Leonor

Pereira, Luiz

Pereira, Maria Carmo

Pereira, Maria De Lourdes

Pereira, Maria G.

Pereira, Nelson A. M.

Pereira, Olívia R.

Pereira, Patricia

Pereira, Renato

Pereira, Rui

Pereira-Leite, Catarina

Pereira-Maia, Elene

Perera, Lalith

Perera, Rushika

Peres, António M.

Perestrelo, Rosa

Perestrelo, Rosa Maria De Sá

Perez Castillo, Yunierkis

Pérez De Vega, María Jesús

Perez Del Real, Rafael

Pérez Pérez, María

Pérez Puyana, Víctor Manuel

Perez Reyes, Carolina

Pérez Sestelo, José

Pérez, Ana G.

Pérez, Antonio

Pérez, Elías

Perez, Juan J.

Perez, Laurent

Perez, Raphael

Perez, Serge

Pérez-Álvarez, Jose Angel

Pérez-Casas, Silvia

Pérez-Gálvez, Antonio

Pérez-García, Selene

Pérez-Jiménez, Jara

Pérez-Lozano, Pilar

Pérez-Mendoza, Manuel J.

Perez-Sayans, Mario

Perfetti, Mauro
Pergher, Sibele B. C.

Périgo, Elio Alberto

Perin, Giorgio

Perinelli, Diego Romano

Perioli, Luana

Peris, José-Esteban

Periyasamy, Palsamy

Perkins-Veazie, Penelope

Perkovic, Ivana

Perlepes, Spyros

Perles, Josefina

Perlikowski, Dawid

Perlin, Michael

Permyakov, Eugene

Perna, Filippo

Perna, Giuseppe

Perni, Michele

Pero, Raffaela

Peron, Gregorio

Perova, Tatiana S.

Perreau, Francois

Perret, Alain

Perrin, Charles L.

Perrin, Lara

Perrone, Daniela

Perrucci, Stefania

Perry, Robin

Persuric, Zeljka

Perteghella, Sara

Perugini, Paola

Perut, Francesca

Pes, Giovanni Mario

Pescina, Silvia

Pessela João, Benevides

Pestov, Alexander

Pestryakov, Alexey

Petcu, Cristian

Petelin, Andrej

Peter, Anca

Peter, Francisc

Peter, Karin

Peter, Martin

Peters, Judith

Peters, Verena

Peterson, Eric Charles

Peterson, Julia

Peterson, Larryn

Petit, Christophe

Petit, Patrice X.

Petkoski, Spase

Petkova, Nadezhda 
Petkovic, Hrvoje

Petkovic, Milena

Petkowski, Janusz J.

Petrásková, Lucie

Petriccione, Milena

Petrikaite, Vilma

Petrillo, Giovanni

Petrini, Marino

Petroni, Katia

Petronilho, Ana

Petropoulos, Spyridon

Petrosino, Maria

Petrou, Christos

Petrov, Dmitry

Petrova, Krasimira T.

Petrović, Biljana

Petrović, Marija

Petrucci, Rita

Petrucci, Roberto

Petruczynik, Anna

Petruzzelli, Gianniantonio

Petruzzi, Leonardo

Petti, Carloalberto

Pettinari, Claudio

Petuhov, Oleg

Petzold, Guillermo

Peuronen, Anssi

Peveler, William

Peyrottes, Suzanne

Peyton, David H.

Pezzani, Raffaele

Pezzato, Luca

Pezzella, Alessandro

Pezzella, Cinzia

Pezzuto, Aldo

Pfattner, Raphael

Pfister, Barbara

Phadtare, Varsha

Pham, Thao

Phan, Chin-Soon

Phan, Thi Tuong Vy

Phapale, Prasad B.

Philipp, Manfred

Philippe, Allan

Philips, Neena

Phillips-Jones, Mary K.

Piao, Xijun

Piasecka, Anna

Piątczak, Ewelina

Piatkevich, Kiryl

Piątkowski, Marek
Picariello, Gianluca

Picchio, Rodolfo

Piccialli, Gennaro

Piccionello, Antonio Palumbo

Piccirillo, Clara

Piccolella, Simona

Picerno, Patrizia

Pichini, Simona

Pichler, Verena

Pichon, Céline

Pickering, Andrew M.

Pickford, Russell

Pico, Yolanda

Picone, Delia

Pidot, Sacha J.

Piechowiak, Tomasz

Pieczywek, Piotr M.

Piedade, Ana Paula

Piegat, Agnieszka

Piekoszewski, Wojciech

Piel, Markus

Pielech-Przybylska, Katarzyna

Pielichowski, Krzysztof

Piemonte, Vincenzo

Piemontese, Luca

Pieraccini, Stefano

Pieretti, Stefano

Piergiovanni, Luciano

Pierini, Filippo

Piermatti, Oriana

Pierson-Wickmann, Anne-Catherine

Pierzynowski, Stefan

Pietropolli Charmet, Andrea

Pietrzyk, Bozena

Pietrzyk, Piotr

Pietrzyk-Brzezinska, Agnieszka J.

Pietschnig, Rudolf

Piga, Isabella

Pigge, F. Christopher

Pigliacelli, Claudia

Pignataro, Bruno

Pignitter, Marc

Piiper, Albrecht

Pike, Nicholas A.

Piktel, Ewelina

Pilarska, Agnieszka A.

Pileio, Giuseppe

Pilepić, Viktor

Pilic, Branka

Pilkington, Lisa

Pilla, Viviane 
Pilling, Darrell

Pilo, Maria I.

Pilo-Pais, Mauricio

Piluso, Susanna

Piluzza, Giannella

Pimienta, Rodney Lacret

Pinakoulaki, Eftychia

Pinalli, Roberta

Pinar, Anita

Pindelska, Edyta

Pineda Rodríguez, Teresa

Pinedo-Rivilla, Cristina

Pinela, José

Pinent Armengol, Montserrat

Piñero, Jose Carlos

Piñero, Manuel

Pingarrón, José

Pinheiro, Marina

Pinho, Luís

Pini, Elena

Pini, Valerio

Pinotti, Luciano

Pintea, Adela

Pinti, Marcello

Pinto, Diana Cláudia

Pinto, Ernani

Pinto, Eugénia

Pinto, Javier

Pinto, Sara

Pintor, Ariana

Pintus, Gianfranco

Pinzaru, Iulia

Pinzi, Luca

Piosik, Jacek

Piotrowska, Urszula

Piotrowski, Paulina

Piotrowski, Tomasz

Piotto, Stefano P.

Piovesan, Damiano

Piper, Brian

Pirani, Fernando

Pires, Regina Helena

Piret, Jocelyne

Piriou, Yannick

Pirman, Tatjana

Pisana, Simone

Pisaneschi, Federica

Pisani, Didier F.

Pishgar, Roya

Pisk, Jana

Piska, Kamil
Pisklak, Dariusz Maciej

Pispas, Stergios

Pitsikalis, Marinos

Pitsinos, Emmanuel

Pittelkow, Michael

Pitto-Barry, Anaïs

Pitucha, Monika

Piubelli, Chiara

Pivac, Nela

Pivetta, Tiziana

Piwowarski, Jakub P.

Pizzi, Antonio

Pizzo, Elio

Pizzuti, Lucas

Placzek, William J.

Plagge, Jan

Plapp, Bryce

Plasseraud, Laurent

Plastina, Pierluigi

Platas-Iglesias, Carlos

Platella, Chiara

Platta, Harald

Platts, James

Plavec, Janez

Plaza, Encarna Gomez

Płaza, Grażyna

Plaza-Díaz, Julio

Plazinski, Wojciech

Plech, Tomasz

Plenis, Alina

Pleshko, Nancy

Pletsa, Vassiliki

Pletschke, Brett

Plíhalová, Lucie

Pliss, Artem M.

Plochberger, Birgit

Płotka-Wasylka, Justyna

Plotniece, Aiva

Pluskal, Tomáš

Pluta, Ryszard

Pluth, Michael D.

Plyushch, Artyom

Pobelov, Ilya

Pochard, Isabelle

Pocheć, Ewa

Pochet, Sylvie

Pocurull, Eva

Poddelsky, Andrey

Podkościelna, Beata

Podlewska, Sabina

Podlipnik, Črtomir 
Podolska, Grazyna

Podsedek, Anna

Podsiadły, Radosław

Podvorica, Fetah

Poeggeler, Burkhard

Poetsch, Ansgar

Pogacnik, Lea

Poggi, Alessandro

Poggio, Claudio

Pogorzelec-Glaser, K.

Pohl, Carolina H.

Pohl, Ehmke

Pohl, Pawel

Pohl, Sebastian

Pohl, Sebastian Öther-Gee

Pohlkamp, Theresa

Pohlmann, Adriana

Poiana, Marco

Poiana, Mariana-Atena

Poinsot, Verena

Póka, Róbert

Poklar Ulrih, Nataša

Pokrywka, Andrzej

Polák, Beata

Polak-Berecka, Magdalena

Poláková, Monika

Polakowski, Nicholas J.

Polanski, Marek

Polčic, Peter

Poletto, Matheus

Poli, Alessandro

Politeo, Olivera

Politis, Ioannis

Polito, Laura

Pollari, Francesco

Pollastro, Stefania

Polosa, Riccardo

Polverino, Pierpaolo

Polyak, Boris

Polyakov, Nikolay

Polz-Dacewicz, Małgorzata

Poma, Paola

Pomastowski, Paweł

Pomerantz, William

Pomin, Vitor

Pon Velayutham, Anandh Babu

Ponce-Vargas, Miguel

Ponczek, Michał B.

Ponik, Suzanne

Ponnandy, Prabhu

Pons, Josefina
Ponti, Alessandro

Pontiki, Eleni

Pontrelli, Paola

Ponzoni, Ignacio

Poon, Gregory

Pop, Anca

Pop, Carmen Rodica

Pop, Cristina

Pop, Laurentiu

Pop, Tudor Lucian

Popa, Daniela-Saveta

Popa, Lacramioara

Popa, Marcel

Popa, Mona Elena

Popa-Wagner, Aurel

Popescu, Radian

Popescu, Vasilica

Popgeorgiev, Nikolay

Pop-Georgievski, Ognen

Poplawski, Tomasz

Popov, Alexey A.

Popov, Anton

Popova, Milena P.

Popović Đorđević, Jelena

Popovich, David

Porat, Ze'ev

Porcelli, Fernando

Poręba, Marcin

Pořízka, Pavel

Porro, Chiara

Portilla, Jaime

Portillo-Estrada, Miguel

Porto, André Luiz Meleiro

Portugal, Camila C.

Poŝa, Mihalj

Poso, Antti

Pospisil, Jiri

Pospisil, Tomas

Possik, Elite

Potaczek, Daniel P.

Potaniec, Bartłomiej

Potapov, Andrei

Potgieter, Martin Johannes

Pothupitiya, Jinal

Potopnyk, Mykhaylo

Poudel, Tej Narayan

Pouli, Nicole

Pouranian, M. Reza

Pourbaghi Masouleh, Milad

Pournik, Maysam

Pous Rodríguez, Narcís 
Poveda Larrosa, Jose Antonio

Powers, Evan

Powers, Robert

Poynter, Matthew E.

Pozio, Alfonso

Pozzi, Cecilia

Pozzi, Gianluca

Prabakaran, Mayakrishnan

Prabhu, Vivek

Pracharova, Jitka

Pradhan, Shantanu

PradoMassarioli, Adna

Pramanik, Arindam

Prandi, Cristina

Pranovich, Andrey

Praplan, Arnaud P.

Prasad, Ram

Prashar, Sanjiv

Prassl, Ruth

Prat, Maria

Pratesi, Alessandro

Pratsinis, Harris

Predoi, Daniela

Preiner, Darko

Preite, Marcelo

Prell, James

Premasiri, Ranjith

Preston, Dan

Pretorius, Sakkie

Pretsch, Thorsten

Prévost, Sébastien

Prhashanna, Ammu

Pricl, Sabrina

Prieto Garcia, Jose

Prieto, Elena

Prieto, Isabel

Prieto, Jose

Prieto, Miguel

Prieto, Miguel Ángel

Prieto-Garcia, Jose M.

Prieto-Lloret, Jesus

Prijić, Aneta

Primožič, Ines

Prisic, Sladjana

Prisinzano, Thomas

Pritzker, Kenneth

Privette Vinnedge, Lisa

Prlić Kardum, Jasna

Probert, Chris

Prochazka, Marek

Prochor, Piotr
Procopio, Jesús R.

Prodanovic, Radivoje

Proestos, Charalampos

Profire, Lenuta

Proietti, Noemi

Prokai, Laszlo

Prokop, Izabela

Prokopov, Tsvetko

Prorokova, Natalia Petrovna

Prosa, Mario

Prosen, Helena

Protti, Michele

Proulx, Caroline

Proulx, Stephanie

Provenzani, Alessandro

Provost, Patrick

Prudêncio, Miguel

Pruna, Alina Iuliana

Pruneanu, Stela-Maria

Prusik, Krystian

Pruvost, Alain

Prywer, Jolanta

Przybył, Jarosław

Przybyłek, Maciej

Przybylski, Piotr

Przybylski, Wiesław

Psomas, George

Psurski, Mateusz

Psycharis, Vassilis

Ptaszek, Anna

Ptaszek, Marcin

$\mathrm{Pu}$, Xiaoping

Pucci, Andrea

Pucek, Agata

Puchades-Carrasco, Leonor

Puchalka, Radoslaw

Pucheault, Mathieu

Puchkova, Ludmila V.

Puchta, Ralph

Puertas-Mejía, Miguel A.

Pugajeva, Iveta

Puiatti, Marcelo

Pulido, Olga M

Pulito, Claudio

Pullano, Salvatore A.

Pulvirenti, Alfredo

Pum, Dietmar

Punta, Carlo

Purcell, Anthony

Purgatorio, Rosa

Puri, Anu 
Purrello, Roberto

Purse, Byron W.

Purves, Randy

Pusch, Stefan

Püschel, Gerhard P.

Pushkar, Yulia

Puszka, Andrzej

Puszkarewicz, Alicja

Putnam, William C.

Putrinš, Marta

Puttreddy, Rakesh

Putz, Mihai

Pyka, Alina

Pyka-Pająk, Alina

Pylypchuk, Ievgen V.

Pyne, Stephen

Pyun, Jae-Cheol

Pyzik, Michal

Qazi, Sohail

Qi, Hang

Qi, Ji

Qi, Yanfei

Qi, Zhou

Qian, Jin

Qian, Jin-yuan

Qian, Mingxing

Qian, Zhao

Qiao, Mingyu

Qiao, Qiquan

Qiao, Yu

Qiaobin, $\mathrm{Hu}$

Qin, Dajun

Qin, Donghuan

Qin, Sanbo

Qin, Xiaoli

Qin, Yu

Qin, Zhihui

Qing, Lin-sen

Qiu, Minghua

Qiu, Qiwen

Qu, Lingbo

$\mathrm{Qu}$, Xiaozhong

Quadrelli, Paolo

Quan, Guilan

Quax, Paul

Queiroz, Maria João

Queneau, Yves

Quesada, Ernesto

Quesne, Matthew

Quevedo, Ivan R.

Quevedo, Roberto
Quijada-Garrido, Isabel

Quijada-Maldonado, Esteban

Quiles Morales, Jose L.

Quilez, Jose

Quiliano, Miguel

Quinet, Pascal

Quinn, Mark T.

Quintard, Adrien

Quintás, Guillermo

Quintas, Luis Eduardo M.

Quintieri, Laura

Quinto, Emiliano José

Quinto, Maurizio

Quirino, Joselito P.

Quiroga, Diego

Quodbach, Julian

Qyyum, Muhammad Abdul

R. El-Seedi, Hesham

Raabe, Gabriele

Raak, Norbert

Raasmaja, Atso

Rabanal Ruiz, Yoana

Rabbani, Gulam

Rabilloud, Franck

Rabu, Pierre

Racané, Livio

Race, Marco

Rachek, Lyudmila I.

Rachwalski, Michał

Racoviceanu, Roxana

Rácz, Anita

Radaram, Bhasker

Radchenko, Eugene V.

Radenkovic, Miroslav

Radenković, Slavko

Radhakrishnan, Ammu K.

Radic, Zoran

Radić, Zoran

Radisavljevic, Ziv

Rádis-Baptista, Gandhi

Radko, Lidia

Radošević-Stašić, Biserka

Radu, Gabriel Lucian

Radulescu, Cristiana

Raduly, Lajos Zsolt

Radušienè, Jolita

Radwan, Mohamed

Rae, Colin

Raffa, Demetrio

Rafieenia, Razieh

Rafińska, Katarzyna 
Rafiński, Zbigniew

Ragab, Khaled

Raganati, Francesca

Ragauskas, Arthur

Ragg, Enzio

Raghavan, Shreya

Ragino, Yuliya I.

Ragnini-Wilson, Antonella

Ragno, Gaetano

Ragno, Rino

Ragucci, Sara

Ragusa, Andrea

Ragusa, Salvatore

Raharivelomanana, Phila

Rahier, Hubert

Rahimi, Farid

Rahimi, Parvaneh

Rahimi-Gorji, Mohammad

Rahman, Arman

Rahman, Ashiqur X.

Rahman, Azizur

Rahman, Faiz

Rahman, M. Azizur

Rahman, Md Toufiqur

Rahme, Kamil

Rai, Ankit

Railean-Plugaru, Viorica

Raimondi, Laura

Raimondi, Lavinia

Raimondi, Maria Valeria

Raimondo, Domenico

Raimundo, Joana

Raimundo, Nuno

Raina, Deepak

Raina, Komal

Rainer, Alberto

Rainey, Jan

Rais, Rana

Rajauria, Gaurav

Rajendiran, Karthikraj

Rajić, Zrinka

Rajkovic, Ivan

Rajnak, Michal

Rajnarayanan, Rajendram

Raju, Ritesh

Rakass, Souad

Rakhmatullin, Aydar

Rakitin, Oleg A.

Ralston, Emily

Rama, Ana Rosa

Ramadan, Sherif
Ramadoss, Ananthakumar

Ramaiahgari, Sreenivasa C.

Ramakrishnan, Sayanthan

Ramalho, Maria João

Ramalho-Santos, Joao

Ramalingam, Nagendran

Ramanavicius, Arunas

Ramani, Komal

Ramasamy, Elamparuthi

Ramasamy, Thiruganesh

Ramil, Carlo

Ramírez, David

Ramírez, Martín

Ramirez-Arcos, Sandra

Ramirez-Gonzalez, Gustavo

Ramis, Gianguido

Rammohan, Aluru

Ramont, Laurent

Ramos, Catarina

Ramos, M. Teresa

Ramos, Marco A.

Ramos, Ricardo Martins

Ramos, Rui Miguel

Ramos, Sonia

Ramos-Organillo, Ángel

Ramos-Ortiz, Gabriel

Ramos-Vivas, José

Rampe, ElizabEth

Rampias, Theodoros

Ran, Chongzhao

Ran, Yan

Ranadheera, Senaka

Ranc, Václav

Rancan, Marzio

Randazzo, Giuseppe Marco

Randazzo, Walter

Rando, Daniela

Ranjan, Amaresh

Ranjan, Atul

Ranzato, Elia

Rao, Ashit

Rao, Ashutosh

Rapa, Mattia

Rapeanu, Gabriela

Râpeanu, Gabriela

Rapone, Biagio

Rapposelli, Simona

Raskovalov, Anton

Raspanti, Mario

Rassias, Gerasimos

Rastija, Vesna 
Rastrelli, Luca

Rasulev, Bakhtiyor

Ratajewski, Marcin

Ratcliffe, Norman

Ratcliffe, Richard George

Rateb, Mostafa

Rather, Irfan

Rathinasabapathy, Anandharajan

Rathore, Atul S.

Ratke, Lorenz

Ratnakumar, Bugga V.

Rattenholl, Anke

Raucci, Umberto

Raudino, Antonio

Raugei, Giovanni

Raúl, Avila-Sosa

Rault, Francois

Ravanidis, Stylianos

Ravi, Rama S.

Ravin, Nikolai V.

Ravula, Sudhir

Rawel, Harshadrai

Rawson, Jeremy

Ray, Asim

Ray, Partha

Rayan, Anwar

Raynie, Douglas

Raza, Aun

Razo, Rodrigo

Razorenov, Dmitry Yurievitch

Re, Suyong

Rea, Ilaria

Réal, Florent

Reany, Ofer

Rébé, Cédric

Reber, Christian

Rebollo-Hernanz, Miguel

Recabarren Gajardo, Gonzalo

Recek, Nina

Redan, Benjamin

Reddy, M. V.

Reddy, Priyanka

Reddy, Sakamuri

Redell, John

Redondo, Pedro Cosme

Redshaw, Carl

Ree, Moonhor

Reeh, Peter

Reekie, Tristan A.

Rees, D. Andrew S.

Reetz, Manfred T.
Reeve, Vivienne

Regazzoni, Luca Giovanni

Rege, Ameya

Regiec, Andrzej

Regmi, Bishnu

Reguero, Mar

Rehder, Dieter

Rehman, Junaid Ur

Rehmann, Holger

Reid, Russell C.

Reid, Scott

Reimann, Regina R.

Reinach, Peter $\mathrm{S}$.

Reis, Catarina

Reis, Catarina Pinto

Reis, Cristiano

Reis, Glaydson Simoes Dos

Reiser, Oliver

Reiss, Lucinda V.

Rejah, Shahi

Relat, Joana

Remedios Serrano, Dolores

Remenyik, Judit

Ren, Baozeng

Ren, Hao

Ren, Qinlong

Ren, Shijie

Ren, Yanrong

Ren, Yulin

Renard, Pierre-Yves

Renaud, Jean-Luc

Renaud, Justin

Renault, Nicolas

Renčiuk, Daniel

Rencoret, Jorge

Rendle, Phillip M.

Rengasamy, Kannan

Renò, Filippo

Rentschler, Eva

Renukuntla, Jwala

Renzo, Vanna

Reščič, Jurij

Rescifina, Antonio

Rescigno, Antonio

Resconi, Virginia Celia

Resendiz, Marino

Reshetilov, Anatoly N.

Restivo, Francesco M.

Reuter, Klaus

Revelou, Panagiota-Kyriaki

Revilla, Eugenio 
Revnic, Cornelia

Revuelta, Julia

Rey, Felisa

Reyes, Archie

Reyes, Fernando

Reyes-Chilpa, Ricardo

Reyes-Ortega, Felisa

Reynisson, Jóhannes

Reza, Khan Mamun

Řezáčová, Martina

Rezaei, Nima

Rezania, Shahabaldin

Řezanka, Michal

Reznikov, Leah R.

Rezvani, Khosrow

Rezzani, Rita

Rhazi, Larbi

Rhee, Ki-Jong

Rhee, Young Ho

Rho, Jung-Rae

Rial, Raquel

Ribaudo, Giovanni

Ribechini, Erika

Ribeiro Macedo, Sandra

Ribeiro, Artur

Ribeiro, Claudia

Ribeiro, Helena

Ribeiro, Luís Filipe

Ribeiro, Miguel

Ribeiro, Thierry

Ricardo Da Silva, Jorge

Ricardo, Fernando

Ricardo, Gobato

Ricardo-da-Silva, Jorge

Riccardi, Claudia

Ricchi, Matteo

Ricci, Arianna

Ricci, Pier Carlo

Riccio, Angelo

Riccò, Raffaele

Rice, Cynthia Ann

Ricelli, Alessandra

Richards, Dylan Jack

Richardson, Alan

Richardson, Rudy J.

Richardson, Simon

Richert, Ranko

Richter, Katrin

Richter, Peter

Richter, Shachar

Richtera, Lukáš
Ridruejo, Álvaro

Riedel, Stefanie

Rigalli, Juan Pablo

Rigamonti, Luca

Rigano, Daniela

Righetti, Laura

Rigoni, Federica

Rijo, Patrícia

Rimbach, Gerald

Rimpelova, Silvie

Rinaldi, Federica

Rincon Joya, Miryam

Rinnerthaler, Mark

Rintoul, Ignacio

Río Segade, Susana

Riobé, François

Rios, Ramon

Riou, Laurent

Ripolles, Teresa S.

Risbridger, Gail

Riscoe, Mike

Risinger, April L.

Rispoli, Concetta

Ristic, Renata

Ritter, Helmut

Ritz, Ulrike

Rivadeneyra, Almudena

Rivas, Carmen

Rivas, Fatima

Rivera De La Rosa, Javier

Rivera, Víctor M.

Rivera-Monroy, Zuly Jenny

Rivero Cruz, José Fausto

Rivero-Pérez, M. Dolores

Rivilla, Rafael

Rizvi, Syed A. A.

Rizzi, Corrado

Rizzi, Laura

Rizzi, Menico

Rizzo, Carla

Rizzo, Fabio

Rizzolio, Flavio

Rizzuti, Bruno

Ro, Hyeon-Su

Roa Rovira, Joan Josep

Robaszkiewicz, Agnieszka

Robert Sosnowski, Tomasz

Robert, Paz

Roberto Da Silva, Edson

Roberts, Arthur G.

Roberts, Stephen K. 
Roberts, Tara

Robertson, Mark J.

Robeyns, Koen

Robien, Wolfgang

Robiette, Raphael

Robinson, Seri

Robledo Quintos, Norma R

Rocca, Roberta

Rocchetti, Gabriele

Rocchigiani, Luca

Rocha, Cláudia

Rocha, Djenisa H. A.

Rocha, Hugo

Rochais, Christophe

Rocha-Martin, Javier

Rochani, Ankit

Roche, Enrique

Roche, Manon

Roche, Michelle

Rochfort, Simone

Rocio-Bautista, Priscilla

Rockenfeller, Patrick

Roda, Gabriella

Rode, Tone Mari

Rodembusch, Fabiano

Rodembusch, Fabiano S.

Rodger, Alison

Rodilla, Vicent

Rodolfo, Carlo

Rodrigo, Luis

Rodrigo, Sara Morales

Rodrigues, Alírio

Rodrigues, Clóves G.

Rodrigues, Francisca

Rodrigues, João

Rodrigues, Luiz Frederico

Rodrigues, Márcio

Rodríguez Díaz, Juan Carlos

Rodríguez González, Álvaro

Rodríguez González, Vicente

Rodríguez López, María Isabel

Rodriguez Rojo, Soraya

Rodríguez Romero, Adela

Rodríguez Villanueva, Javier

Rodriguez, Guillermo

Rodriguez, Hortensia M.

Rodriguez, Jaime

Rodríguez, José Antonio

Rodríguez, Laura

Rodríguez, Mario

Rodríguez, Ricardo Reyes
Rodriguez-Couto, Susana

Rodríguez-Flores, M. Shantal

Rodríguez-López, Carlos E.

Rodriguez-Lorenzo, Laura

Rodríguez-Roque, María Janeth

Rodriguez-Sinovas, Antonio

Rodríguez-Yoldi, María Jesús

Rodriquez, Hector

Rodriquez, Manuela

Rodziewicz-Motowidło, Sylwia

Roedel, Franz

Roex, Erwin

Rofouie, Pardis

Roger, Jean Michel

Roger, Julien

Roglans, Anna

Rogobete, Alexandru Florin

Rogozhin, Eugene

Roh, Changhyun

Roh, Jaroslav

Rohanifar, Ahmad

Rohde, Kyle

Rohlena, Jakub

Rohn, Sascha

Röhrig, Ute

Roj, Edward

Rojas, Claudia

Rojas-Aguirre, Yareli

Rojas-Hernandez, Rocío Estefanía

Rojo, Maria Angeles

Rokavec, Matjaz

Rokosz, Krzysztof

Rolfe, Barbara E.

Rolim, Adriana

Rolle, Luca

Rollin, Patrick

Rolo, Joana

Román Guerrero, Angélica

Roman, Celian

Romani, Andrea

Roman-Junior, Walter

Romano, Giovanna

Romański, Jarosław

Romar, Henrik

Roma-Rodrigues, Catarina

Romeo, Isabella

Romeo, Roberto

Romero, Alejandro

Romero, Marta R.

Romero, Rosenberg J.

Romero-González, Roberto 
Romero-Muñiz, Carlos

Romero-Salguero, Francisco J.

Romoli, Ottavia

Ronca, Alfredo

Roncada, Paola

Rončević, Sanda

Ronchetti, Simona

Ronconi, Luca

Rondeau-Gagné, Simon

Rondon, Aurélie

Ronquist, Gunnar

Ronzan, Marilena

Roose, Bart

Roosen-Runge, Felix

Root, Martin

Roper, Stephen D

Roppolo, Ignazio

Roque, Alicia

Roques, Jonathan

Rosa Da Silva, Robson

Rosa, Antonella

Rosa, Arianna Carolina

Rosa, Marco

Rosado, Catarina

Rosales, Julia

Rosales-Hernández, Martha Cecilia

Roscini, Luca

Rosenau, Thomas

Rosenberg, Erwin

Rosenberg, Ivan

Rosenberg, Moshe

Rosenberry, Terrone

Rosenthal, Katrin

Rosenzweig, Derek

Rosi, Marzio

Rosicka-Kaczmarek, Justyna

Rosini, Elena

Rosokha, Sergiy V.

Ross, Ian L

Ross, Kirstin

Ross, Robert L.

Ross, Thyer

Ros-Santaella, Jose Luis

Rossella, Dorati

Rossella, Francesco

Rossi, Claudio

Rossi, Filippo

Rossi, Filomena

Rossi, Giancarlo

Rossi, Jean-Christophe

Rossi, Marco
Rossi, Miriam

Rossi, Sergio

Rostoker, Guy

Roszak, Szczepan

Roszkowska, Anna

Rotaru, Andrei

Rotblat, Barak

Roterman, Irena

Roth, Michael

Rotondo, Archimede

Rotstein, Benjamin $\mathrm{H}$.

Roubaud, Anne

Roubelakis-Angelakis, Kalliopi A.

Rouphael, Youssef

Roussis, Ioannis G.

Rout, Bhimsen

Rovini, Amandine

Rovisco, Ana

Rovnak, Joel

Row, Kyung Ho

Rowlands, Gareth J.

Rowles, Matthew

Roy Chowdhury, Sandipan

Roy, Anuradha

Roy, Dhruvajyoti

Roy, Dipankar

Roy, Juganta

Roy, Renè

Różalska, Barbara

Różalska, Sylwia

Rozalski, Marcin

Rozès, Nicolas

Rozhon, Wilfried

Rožić, Mirela

Rozwadowski, Tomasz

Różycki, Bartosz

Różyło, Renata

Rozza, Ariane

Ruan, Ke

Ruaro, Barbara

Rubakhin, Stanislav

Rubčić, Mirta

Rubino, Federico Maria

Rubio Alonso, Fausto

Rubio Alonso, Juan

Rubio-Martinez, Jaime

Rubis, Blazej

Rudat, Jens

Ruddraraju, Kasi Viswanatharaju

Rudić, Svemir

Rudick, Jonathan 
Rüdiger, Stefan

Rudiuk, Sergii

Rudnicka, Wiesława

Rudnóy, Szabolcs

Rudshteyn, Benjamin

Rudzińska, Magdalena

Rueda Ordóñez, Yesid Javier

Ruegg, Urs

Ruelland, Eric

Ruffino, Francesco

Ruffolo, Gabriele

Ruiu, Luca

Ruiz Téllez, Trinidad

Ruiz, Mario

Ruiz-Angel, Maria José

Ruiz-Rubio, Leire

Ruiz-Sánchez, Esaú

Rulíšek, Lubomír

Ruml, Tomas

Rumlová, Michaela

Rumyantseva, Marina

Rupasinghe, Thusitha

Ruperez, Francisco J.

Ruphuy, Gabriela

Ruppert, Romain

Rusciano, Maria Rosaria

Rusike, Constance

Rusinek, Robert

Rusmini, Paola

Rusnati, Marco

Russell, Fraser

Russo Krauss, Irene

Russo, Alessandra

Russo, Laura

Russo, Luigi

Russo, Mariateresa

Russo, Marina

Russo, Mario Vincenzo

Russo, Nino

Russo, Pasquale

Russo, Pietro

Russo, Roberta

Russo, Rosita

Russo, Teresa

Russu, Wade

Rusu, Daniela

Ruthstein, Sharon

Rutkowska, Małgorzata

Ruysschaert, Jean-Marie

Ruzik, Lena

Ruzza, Chiara
Ryabchikova, Elena I.

Ryabchun, Alexander

Ryadnov, Max

Ryan, Ali

Rybak, Katarzyna

Rybarczyk, Piotr

Rybarczyk-Pirek, Agnieszka J.

Rybczyńska-Tkaczyk, Kamila

Rybka, Jakub

Ryde, Ulf

Rykowska, Iwona

Ryo, Ushioda

Ryoo, Sungweon

Ryu, BoMi

Ryu, Jae-Ha

Ryu, Ji Hyun

Ryu, Seung Yoon

Rywaniak, Joanna

Ryzhov, Victor

Rzelewska-Piekut, Martyna

Sa, Jeong-Hoon

Saaby, Lasse

Saad, Jamil

Saada, Ann

Saba, Sumbal

Sabantina, Lilia

Sa-Barreto, Livia L.

Sabaté, Raimon

Sabater i Serra, Roser

Sabatini, Jesse J.

Sabatini, Stefano

Sabatino, David

Sabatino, Manuela

Sabir, Shekh

Sabiu, Saheed

Saboe, Patrick

Sabol, Ivan

Šabovič, Mišo

Sabri, Firouzeh

Saccenti, Edoardo

Sacchetti, Alessandro

Sacco, Riccardo

Saccone, Salvatore

Sachadyn-Król, Monika

Sacher, Edward

Sachse, Alexander

Sacks, Gavin

Sackus, Algirdas

Sacré, Pierre-Yves

Saczonek, Agnieszka Owczarczyk

Sadeghi, Saman 
Sadeghpour, Amin

Sadgrove, Nicholas

Sadhasivam, Sudharsan

Sadjadi, SeyedAbdolreza

Sadovskaya, Irina

Sadowska, Anna

Sadowska, Monika

Sadowska-Krępa, Ewa

Saeb, Mohammad Reza

Saeki, Kumiko

Safa, Ahmad R.

Safe, Stephen H.

Šafratová, Marcela

Sagar, Robin P.

Sagnou, Marina

Sagona, Simona

Sah, HongKee

Saha, Manik Lal

Saha, Rajib

Saha, Subhasish

Sahoo, Sanjubala

Sahu, Ravi P

Saiano, Filippo

Saiardi, Adolfo

Saidaminov, Makhsud

Saielli, Giacomo

Saifutdinov, Bulat R.

Saikat, Bala

Saiko, Guennadi

Saikova, Svetlana

Sainlos, Matthieu

Saint-Fort, Roger

Saito, Akiko

Saito, Kan

Saito, Masaichi

Saito, Satoshi

Saito, Shinichi

Saito, Yoshinori

Saitta, Marco

Sajeva, Maurizio

Sajnóg, Adam

Sakač, Nikola

Sakai, Nobuyuki

Sakai, Ryuichi

Sakamoto, Kathleen M.

Sakamoto, Masahiro

Sakamoto, Takeshi

Sakamuro, Daitoku

Sakar, Mohan

Sakata, Masahiro

Sakilam, Satish
Sakkas, Hercules

Sakurai, Kazumasa

Sakurai, Tsuneaki

Salachna, Piotr

Salafranca Lázaro, Francisco J.

Salahub, Dennis

Saláková, Alena

Salamanca, Constain H.

Salanță, Liana Claudia

Salas, Carlos

Sałat, Kinga

Salaün, Fabien

Salazar, Juan Rodrigo

Saldanha, Carlota

Saleh, Mahmoud

Saleh-E-In, Md. Moshfekus

Salerno, Loredana

Sales, Rita De Cássia Mendonça

Salhab, Hassan

Šalić, Anita

Salman, Mootaz

Salmaso, Veronica

Salmerón, Ivan

Salmon, Eric

Salo-Ahen, Outi

Salomone, Antonio

Salonen, Laura

Salopek-Sondi, Branka

Saltas, Vassilios

Salto-Gonzalez, Rafael

Salvado, Victoria

Salvarani, Nicolò

Salvatori, Roberta

Salvatori, Stefano

Salvetti, Anna

Salvi, Anna M.

Salvo, Andrea

Samanidou, Victoria

Samardžić, Jelena

Samarov, Artemiy A.

Sambri, Andrea

Šamec, Dunja

Samek, Ota

Samhan-Arias, Alejandro

Samková, Eva

Sammalkorpi, Maria

Sampaio, Ana

Sampath, Chethan

Samperi, Filippo

Sampson, Nicole S.

Samson, Willis 
Samsonov, Sergey

San Fabian, Emilio

San Miguel, Veronica

Sanchez Galan, Javier

Sánchez, Goar

Sánchez, Jesus Lozano

Sanchez, Jose M.

Sanchez, Raquel

Sánchez-Barba, Luis F.

Sánchez-Fidalgo, Susana

Sánchez-García, David

Sánchez-Gómez, Rosario

Sánchez-Martín, María Jesús

Sánchez-Monedero, Miguel

Sanchez-Pastor, Enrique

Sanchez-Salas, Jose

Sánchez-Soto, Luis L.

Sánchez-soto, Pedro

Sánchez-Vergara, María Elena

Sancho-Garcia, Juan-Carlos

Sancineto, Luca

Sancini, Giulio

Sandalio, Luisa María

Sandeep, Dave

Sandhu, Amandeep

Sandhu, Bhupinder

Sandhu, Jagdeep K.

Sandjo, Louis Pergaud

Sandlers, Yana

Sándor, Kristyán

Sándor, Zoltán

Sandra, Beaufort

Sandu, Andrei Victor

Sang, Yongming

Sangiovanni, Davide

Sangiovanni, Enrico

Sani, Rajesh

Sanina, Nina M.

Sanjeet, Mehariya

Sanjeewa, Kalu Kapuge Asanka

Sanjurjo Sanchez, Jorge

Sanjust, Enrico

Sankaranarayanan, Nehru Viji

San-Miguel, Adriana

Sanna, Gavino

Sanna, Nico

Sanner, Michel F.

Sannino, Filomena

Sanny, Charles

Sans, Albert

Sansbury, Brian E.
Sansone, Anna

Sansone, Francesca

Sansone, Francesco

Santacruz Ortega, Hisila Del Carmen

Santagapita, Patricio

Santamaria, Pietro

Santamaria, Rita

Santamaria-Echart, Arantzazu

Santana, Andrés González

Santarem, Nuno

Santhakumar, Abishek Bommannan

Santhoshkumar, Puttur

Santi, Claudio

Santilio, Angela

Santin, Silvana M. O.

Santini, Antonello

Santini, Carlo

Santisteban, Maria

Santoro, Fabrizio

Santos De Gois, Jefferson

Santos Júnior, Aníbal De Freitas

Santos Mendes, Luis Felipe

Santos, Carla S.

Santos, Clementina

Santos, Cleydson Breno R.

Santos, Eva M.

Santos, Maria Amélia

Santos, Maria M. M.

Santos, Raquel De Cássia Dos

Santos, Ronaldo G.

Santos, Sonia A.O.

Santos, Susana S.

Santos, Willy Glen

Santos-Filho, Osvaldo Andrade

Santovito, Gianfranco

Santra, Biswajit

Santucci, Annalisa

Sañudo-Barajas, Josefa Adriana

Sanz-Marco, Amparo

Saponara, Simona

Sarabia, Francisco

Saraiva, Nuno

Sarandeses, Luis A.

Sarasini, Fabrizio

Saravana, Periaswamy Sivagnanam

Saravanakumar, Kandasamy

Sardone, Rodolfo

Saretzki, Gabriele

Šarkanj, Bojan

Sarkar, Amit Kumar

Sarkar, Dhruba 
Sarlis, Nicholas

Sarmento-Ribeiro, Ana Bela

Sarno, Federica

Sartorelli, Patricia

Sasai, Yasushi

Sasaki, Nobuo

Sasaki, Sergio Daishi

Sashuk, Volodymyr

Sassi, Mohamed

Sastre, Ana Maria

Sastre-Santos, Angela

Sastry, Jagannadha

Satake, Honoo

Satake, Masayuki

Šatínský, Dalibor

Satish, Lakkakula

Sato, Daisuke

Sato, Kazuyuki

Sato, Kenji

Sato, Kousuke

Sato, Kyotaka

Sato, Masamitsu

Sato, Michio

Sato, Shinobu

Sato, Yusuke

Satoh, Shigeru

Satora, Pawel

Sator-Katzenschlager, Sabine

Satou, Tadaaki

Satriano, Cristina

Saturnino, Carmela

Satyal, Prabodh

Sauer, Daniel

Sauka, Diego

Saurav, Kumar

Saurina, Javier

Savage, Kaye

Savage, Paul

Saverwyns, Steven

Saviano, Michele

Savic, Ivan M.

Savić, Jelena

Savina, Irina

Savion, Naphtali

Savoie, Jean-Michel

Savovic, Svetislav

Savy, Davide

Sawa, Teiji

Sawada, Jun-ichi

Sawai, Hirofumi

Sawai, Jun
Sawama, Yoshinari

Sawicka, Barbara

Sawicki, Tomasz

Sayago, Ana

Sayago, Francisco J.

Sayydi, Nima

Sberveglieri, Veronica

Sbirrazzuoli, Nicolas

Scafato, Patrizia

Scafuri, Bernardina

Scala, Angela

Scala, Valeria

Scalise, Mariafrancesca

Scalliet, Camille

Scampicchio, Matteo

Scanu, Antonio Mario

Scarano, Antonio

Scarpa, Emanuele-Salvatore

Scarpa, Marina

Scarso, Alessandro

Scazzina, Francesca

Schab Balcerzak, Ewa

Schabowicz, Krzysztof

Schäfer, Edgar

Schander, Andreas

Schatz, Jürgen

Scheggi, Simona

Scheiner, Steve

Schelhaas, Sonja

Schelvis, Johannes

Schemmer, Peter

Schennach, Robert

Schenone, Silvia

Scherf, Katharina

Scheunemann, Matthias

Schicho, Rudolf

Schiemann, Olav

Schild, Axel

Schilling, Stephan

Schiraldi, Chiara

Schiraldi, David

Schirhagl, Romana

Schirrmacher, Ralf

Schito, Anna Maria

Schlaepfer, Isabel

Schlafer, Sebastian

Schlettwein, Derck

Schmeda-Hirschmann, Guillermo

Schmid, Jochen

Schmidt, Beata

Schmidt, Bernd 
Schmidt, Bernhard V. K. J.

Schmidt, Claudia

Schmidt, Martin

Schmidt, Martina

Schmidt, Walter F.

Schmidtke, Gunter

Schmitt, Tomas

Schmitz, Christian

Schmitz, John C.

Schmitz, Ulf

Schmutz, Marc

Schnabel, Thomas

Schneck, Emanuel

Schneckenburger, Herbert

Schneider, Bernd

Schneider, Ioan

Schneider, Petra

Schneider, Raphael

Schneider, Sabine

Schneider-Schaulies, Juergen

Schnepf, Andreas

Schniepp, Hannes

Schoffstall, Allen

Schohn, Hervé

Schöllhorn, Bernd

Scholtz, Vladimír

Scholz, Carmen

Scholze, Alexandra

Schomacher, Lars

Schorzman, Allison N.

Schraermeyer, Ulrich

Schramm, Michael P.

Schreiber, Michael

Schripsema, Jan

Schröder, Uwe

Schroeder, Grzegorz

Schuchardt, Mirjam

Schughart, Klaus

Schulze, Johannes

Schulzke, Carola

Schulzová, Věra

Schumacher, Udo

Schurig-Briccio, Lici

Schwalbe, Matthias

Schwalbe, Ruth A.

Schwaminger, Sebastian

Schwan, William

Schwans, Jason P.

Schwarz, Dana

Schwarz, Karlheinz

Schwarz, Mónica
Schwarz, Patrick

Schwarz, Simona

Schweiker, Stephanie

Schwendt, Marek

Schwikkard, Sianne

Schwinefus, Jeff

Schymura, Stefan

Sciacca, Michele Francesco Maria

Ścibisz, Iwona

Scicchitano, Pietro

Sciortino, Giuseppe

Sciortino, Maria Teresa

Scipione, Luigi

Sciubba, Fabio

Scoffield, Jessica

Scoffone, Viola Camilla

Scognamiglio, Monica

Scollary, Geoff

Scotece, Morena

Scott, Iain

Scott, John Ashley

Scott, Peter

Scrima, Mario

Scrimin, Paolo

Scudiero, Olga

Scuruchi, Michele

Sczepanski, Jonathan

Sebastian, Aimy

Šebela, Marek

Šebesta, Radovan

Sebestik, Jaroslav

Sebilo, Mathieu

Seca, Ana M. L

Seca, Ana Maria Loureiro Da

Secenji, Aleksandar

Secondo, Agnese

Sedic, Mirela

Sedlak, Milos

Seelam, Prem Kumar

Seene, Teet

Segarra-Marti, Javier

Šegota, Suzana

Seguí Gil, Lucía

Segundo, Marcela

Seidel, Rüdiger W.

Seidl, Claudia

Seifitokaldani, Ali

Seijas Vázquez, Julio A.

Seipke, Ryan

Seisenbaeva, Gulaim

Sekara, Agnieszka 
Sekatski, Serguei

Sekijima, Masakazu

Sękowski, Szymon

Sekutor, Marina

Selene, Sepulveda-Guzman

Selling, Gordon

Selmani, Atiđa

Selvaraju, Ram Kumar

Semenova, Maria G.

Semenyuk, Pavel

Semeril, David

Semreen, Mohammad H.

Semsarilar, Mona

Sen, Rwik

Senesi, Giorgio

Senesi, Nicola

Senge, Mathias

Şenilă, Marin

Senkovska, Irena

Sensini, Alberto

Seo, Dong-Kyun

Seo, Ho Seong

Seo, Hyun Ook

Seo, Soonmin

Sepcic, Kristina

Sepe, Lucia

Sequeda-Castañeda, Luis G.

Sequeira, César A. C.

Serafín, Verónica

Seravalli, Javier

Serefko, Anna

Sereikaite, Jolanta

Sereno, Denis

Serezhkin, Viktor N.

Sergeant, Kjell

Sergeev, Yuri

Sergeyev, Vladimir

Sergi, Consolato

Sergio, Lucrezia

Serio, Annalisa

Serpooshan, Vahid

Serra, Raffaele

Serra, Stefano

Serrador Peiró, Juan M.

Serralheiro, Maria Luísa

Serrano, Dolores R.

Serrano, María

Serrano, Mario

Serrano-Aroca, Ángel

Serreli, Gabriele

Sertić, Miranda
Servili, Maurizio

Sessini, Valentina

Sestili, Francesco

Sestito, Simona

Seto, Edward

Seto, Shigeki

Setyaningsih, Widiastuti

Setzer, William N.

Seung-Hoon, Baek

Sevastre, Bogdan

Severino, Beatrice

Sevic, Dragutin

Sevilla, Michael

Sevilla-Morán, Beatriz

Sewani-Rusike, Constance

Sgarbossa, Paolo

Sgherri, Cristina

Sgorbini, Barbara

Sgouros, Aristotelis

Sgrignani, Jacopo

Sgroi, Mauro Francesco

Shabaninejad, Mehdi

Shabatina, Tatyana

Shafahi, Maryam

Shah, Jindal K.

Shahid, Saroash

Shaik, Anver Basha

Shaik, Mohammed Rafi

Shakeel, Faiyaz

Shakhtshneider, Tatyana P.

Shakibaei, Mehdi

Shakya, Viplendra P. S.

Shalaby, Mohamed

Shan, Wenqian

Shang, Chaoqun

Shang, Qingsen

Shang, Zhuo

Shankar, Eswar

Shankhari, Pritam

Shanmugam, Muruganandan

Shao, Fangwei

Shao, Hua

Shao, Huaiyu

Shao, Longquan

Shao, Lu

Shao, Xueguang

Shao, Yihan

Shapiro, Bruce

Sharan, Shyam

Sharker, Shazid Md.

Sharma, Abhisheak 
Sharma, Ashutosh

Sharma, Geetanjali

Sharma, Isha

Sharma, Kavita

Sharma, Prashant

Sharma, Priyanka

Sharma, Ritin

Sharma, Virag

Sharpe, Shaun M.

Shateyi, Stanford

Shaul, Yoav D.

Shavandi, Amin

Shaw, Lisa

Shaw, Subrata

Shaya, Janah

Shchyogolev, Sergei

Shears, Sthephen

Sheats, Katie

Sheen, Horn-jiunn

Sheidaei, Azadeh

Sheldon, Roger

Sheldrake, Gary

Sheldrake, Helen

Shellaiah, Muthaiah

Shen, Bin

Shen, Jiangchuan

Shen, Jian-ying

Shen, Liming

Shen, Qiang

Shen, Weifeng

Shen, $\mathrm{Xi}$

Shen, Xiao

Shen, Xinggui

Shen, Xiulong

Shen, Yi

Shen, Yiou

Shen, Yudong

Shen, Yuemao

Shen, Zhiqiang

Shenashen, Mohamed A.

Shenderovich, Ilya G.

Sheng, Rong

Sheng, Ruilong

Sheng, Yue

Shenoy, Priyank

Sherwood, James

Sheth, Bhavwanti

Shevtsov, Maxim

Shi, Haitao

Shi, Lei

Shi, Shicheng
Shi, Wen

Shi, Yang

Shi, Yusheng

Shi, Zengqian

Shiao, Young-Ji

Shiau, Lieding

Shiba, Fumiyuki

Shiba, Kiyotaka

Shibaev, Petr

Shibata, Ikuya

Shibatomi, Kazutaka

Shibayama, Nobuko

Shibuya, Masatoshi

Shieh, Tzong-Ming

Shields IV, C. Wyatt

Shiga, Takuya

Shiina, Marisa

Shikov, Alexander

Shikov, Alexander N.

Shimada, Ken-ichiro

Shimada, Masako

Shimada, Yasuhito

Shimanouchi, Toshinori

Shimazu, Shogo

Shimba, Shigeki

Shimizu, Flavio

Shimizu, Makoto

Shimizu, Soji

Shimpei, Iikuni

Shimura, Tatsuo

Shin, Dongyun

Shin, Hyun-Sang

Shin, Jae-Ho

Shin, Soyoung

Shin, Young G.

Shinada, Tetsuro

Shinde, Arti V.

Shinde, Surendra Krushna

Shinoda, Yo

Shinohara, Mitsuru

Shioda, Norifumi

Shioda, Tatsuo

Shiotari, Akitoshi

Shipley, Paul

Shiragami, Tsutomu

Shirahata, Tatsuya

Shirai-Matsumoto, Keiko

Shirakami, Yohei

Shirasuna, Koumei

Shiri-Sverdlov, Ronit

Shivange, Amol 
Shiwarski, Daniel J.

Shnyder, Steve

Shoda, Makoto

Shoemaker, Charles

Shoji, Mitsuo

Shoji, Yoshiaki

Shornick, Laurie

Shoseyov, Oded

Shpigelman, Avi

Shrestha, Namita

Shu, Xinhua

Shuck, Christopher

Shui, Hao-ai

Shuichi, Mori

Shukitt-Hale, Barbara

Shults, Elvira E.

Shun, Yao

Shurpik, Dmitriy

Si, Ruizhe

Siamidi, Angeliki

Siamwala, Jamila H.

Siatka, Tomáš

Šic Žlabur, Jana

Sicari, Vincenzo

Siciliano, Carlo

Sicinski, Rafal

Siddique, Abu Bakar

Sideratou, Zili

Sidorov, Pavel

Sidorova, Yulia

Sieniawska, Elwira

Sier, Cornelis

Sieradzan, Adam

Sierecki, Emma

Sieroslawska, Anna

Sierra-Campos, Erick

Sievänen, Elina

Siewert, Bianka

Sigala, Sandra

Signore, Giovanni

Signorini, Cinzia

Sikalidis, Angelos

Sikora, Janusz

Sikorska, Emilia

Sikorski, Aleksander

Sikorski, Artur

Silacci, Paolo

Silberring, Jerzy

Šiler, Branislav

Šilha, David

Siligardi, Giuliano
Silina, Yuliya

Silipo, Alba

Siliqi, Dritan

Silva Filho, Edson

Silva Moura, Elisabete Ferreira

Silva, Adriana Ribeiro

Silva, Amelia M.

Silva, Artur

Silva, Carlos Alberto

Silva, Catarina

Silva, Catarina L.

Silva, Dr. Márcio S.

Silva, Filomena

Silva, Ines Vieira Da

Silva, Maria Fátima

Silva, Nuno A.

Silva, Paula

Silva, Rui

Silva, Saimon Moraes

Silva, Severiano R.

Silva, Vera L. M.

Silva-Espinoza, Brenda A.

Silvagno, Francesca

Silva-Martínez, Susana

Silveira, Nádya P. Da

Silvestre, Armando J. D.

Silvestre, Samuel

Silvestri, Cristoforo

Sim, Jaehoon

Sim, Uk

Šimáček, Pavel

Simal, Jesus

Šimenas, Mantas

Simeoli, Raffaele

Simeonov, Vasil

Simes, Dina

Simion, Monica

Simirgiotis, Mario

Simko, Fedor

Simmons, Katie

Simões, Pedro

Simões, Zilá Luz Paulino

Simon, Istvan

Simon, Jozsef

Simon, Julian

Simon, Peter

Simonato, Barbara

Simončič, Barbara

Simone, Angela De

Simone, Vincenzi

Simonescu, Claudia Maria 
Simonetti, Giovanna

Simoni, Francesco

Simonpietro, Agnello

Simova-Stoilova, Lyudmila Petrova

Simpson, Bradley S.

Simpson, Thomas J.

Sims, Gerald

Simserides, Constantinos

Sin, Jon

Sinanoglou, Vassilia

Sinclair, Andrew

Singaraju, Aditya

Singer, Steven

Singh, Alok

Singh, Anil

Singh, Anirudha

Singh, Bir Bahadur

Singh, Gurwinder

Singh, Kamalendra

Singh, Kulbir

Singh, Mogie

Singh, Nisha

Singh, Parvesh

Singh, Priyanka

Singh, Ranjeet

Singh, Ruchira

Singh, Rupesh Kumar

Singh, Santosh Kumar

Singh, Sharda P.

Singh, Somnath

Singh, Surya P.

Singha, Subhankar

Singla, Bhupesh

Sinha, Sudarson Sekhar

Sinha-Ray, Suman

Sinjari, Bruna

Šinko, Goran

Sinn, Gerhard

Sintsova, Oksana

Sinyukov, Alexander

Siodłak, Dawid

Siopa, Filipa

Sipos, Emese

Sipos, Katalin

Sipos, Pál

Sippl, Wolfgang

Siracusa, Rosalba

Siramshetty, Vishal B.

Sirbu, Ovidiu

Siren, Heli

Siskos, Michael G.
Sistla, Srinivas

Sita, Giulia

Sitarek, Przemysław

Sithole, Bruce

Sivaev, Igor B.

Sivalingam, Periyasamy

Sivathanu, Vivek

Sizochenko, Natalia

Skaf, Dorothy W.

Skała, Ewa

Skalicka-Woźniak, Krystyna

Skalicky, Milan

Skalniak, Lukasz

Skalova, Lenka

Skaltsa, Liana

Skandalis, Nikolaos

Skarżewski, Jacek

Skelly, Deanne

Skenderidis, Prodromos

Skerget, Mojca

Skibiński, Robert

Skoko, John J.

Skonieczna, Magdalena

Skórczewska, Katarzyna

Skorik, Yury A.

Skouta, Rachid

Skrabana, Rostislav

Skrypnik, Liubov

Skrypnik, Liubov N.

Skrzypczak-Pietraszek, Ewa

Skurnik, Mikael

Skuza, Lidia

Skwarek, Ewa

Slama, James T.

Slaninová, Iva

Sleigh, James

Slim, Azouzi

Slim, Smaoui

Slimestad, Rune

Śliwa, Izabela

Śliwińska-Wilczewska, Sylwia

Sliwinski, Tomasz

Sliwka, Hans-Richard

Sloan, Kenneth

Slobodnikova, Livia

Słoczyńska, Karolina

Słomińska-Wojewódzka, Monika

Slominski, Andrzej

Słomkowski, Stanislaw

Sloop, Joseph

Slowinska, Katarzyna 
Ślusarczyk, Sylwester

Smailyte, Giedre

Šmejkal, Karel

Smeriglio, Antonella

Smet, Mario

Smet, Philippe

Smiesko, Martin

Śmigielski, Krzysztof B.

Smiljanić, Katarina

Smirnov, Nickolay N.

Smith, Jennifer

Smith, Kerry

Smith, Lloyd M.

Smith, Mark

Smith, Micholas Dean

Smith, Zachary

Smulko, Janusz

Smyk, Bogdan

Smyth, James

Snapper, Marc

Snegur, Lubov

Snežana, Papović

Snopek, Lukas

Snopiński, Przemysław

Snow, Nicholas

Snow, Nicholas H.

Snowden, Timothy

Snyder, Nicole

Soares, Ana Raquel

Soares, Bluma G.

Soares, Maria Isabel L.

Soares, Pedro

Šob, Mojmír

Sobczak, Paweł

Sobczak-Kupiec, Agnieszka

Sobeh, Mansour

Sobiesiak, Magdalena

Sobkowski, Michal

Sobolev, Arkadij

Sobotta, Łukasz

Socaci, Sonia

Socaciu, Carmen

Socała, Katarzyna

Socas-Rodríguez, Bárbara

Socha, Katarzyna

Socha, Robert

Sochaj-Gregorczyk, Alicja Maria

Socol, Marcela

Söderberg, Björn C. G.

Soe, Kent

Soengas, Raquel G.
Soesoo, Alvar

Soeta, Takahiro

Sogawa, Chiharu

Sogkas, Georgios

Sohrabi, Salman

Soica, Codruta

Sokolova, Maria Petrovna

Sokolová, Romana

Solache-Ríos, Marcos José

Šolaja, Bogdan A.

Solano, Francisco

Solè, Daniel

Soler, Miguel Ángel

Solieri, Lisa

Soliman, Mahmoud

Soliman, Sameh S. M.

Solís, Luis Fernando Magaña

Sołoducho, Jadwiga

Solomon, Melani

Solomonov, Boris

Solomonov, Inna

Soloshonok, Vadim

Solovieva, Svetlana

Solovyev, Nikolay

Solyanikova, Inna

Solymar, Margit

Somavat, Pavel

Sommerer, Nicolas

Somoza, Álvaro

Somsák, László

Son, Ki-Ho

Son, Seunguk

Sonavane, Manoj

Soncin, Francesca

Sone, Hideko

Song, Baoan

Song, Jianwei

Song, Jianxun

Song, Jong-Won

Song, Juntae

Song, Minjung

Song, Xuezheng

Song, Yang-Heon

Song, Yuanda

Song, Zhiyi

Song, Ziyuan

Sonnenberg, Anton

Sonnier, Rodolphe

Sonowal, Himangshu

Soond, Surinder M.

Soran, Maria-Loredana 
Sordello, Fabrizio

Sørensen, Jens Laurids

Sorensen, John

Soreq, Hermona

Sorg, Olivier

Soria, Ana Cristina

Soriano, José Miguel

Sorokin, Andrey

Sorokina, Irina V.

Sorrentino, Andrea

Sorrentino, Nicolina Cristina

Sosa Díaz, Teresa

Sosic, Izidor

Sosnowska, Dorota

Sosnowska, Katarzyna

Sosnowski, Marcin

Sotelo Mundo, Rogerio

Sotiriou, Georgios

Soto, Alejandro Presa

Soto, Carmen

Soto-Hernández, Marcos

Sotomayor, Nuria

Sottero, Barbara

Sou, Keitaro

Soukoulis, Christos

Soural, Miroslav

Sousa, Carmen

Sousa, Joana L. C.

Sousa, Maria João

Sousa, Sérgio F.

Sousa, Sérgio Filipe

Sova, Matej

Sovadinova, Iva

Soveral, Graça

Sovinco, Fabio

Sowa, Ireneusz

Spaccini, Riccardo

Spadaccini, Roberta

Spagnolo, Vincenzo

Spagnuolo, Carmela

Spain, Jim C.

Spallarossa, Andrea

Spanedda, Maria

Spasov, Alexander A.

Spassov, Tony

Spatari, Sabrina

Specht, Alexandre

Spelzini, Darío

Spencer, John

Spengler, Gabriella

Spetea, Mariana
Spieckermann, Florian

Spiegel, Martin

Spiegler, Verena

Spiers, Andrew

Spingler, Bernhard

Spínola, Vítor

Spiridigliozzi, Luca

Spirina, Liudmila V.

Spivak, David

Spiwok, Vojtech

Spizzirri, U. Gianfranco

Splichalova, Alla

Splinter, Robert

Spohr, Klaus Michael

Sponer, Judit

Spratt, Donald E.

Spur, Bernd W.

Spyroulias, Georgios

Squillacioti, Caterina

Squillaro, Tiziana

Średnicka-Tober, Dominika

Sreejith, Kamalalayam Rajan

Sreerama, Lakshmaiah

Sresht, Vishnu

Srinivas, Keerthi

Sripathy, Smitha

Srirangan, Kajan

Srivastava, Pranay

Srivatsan, Malathi

Sroda-Pomianek, Kamila

Sroka, Zbigniew

Sroka-Bartnicka, Anna

St. Louis, Irina

Stacchiotti, Alessandra

Stachowska, Ewa

Stączek, Sylwia

Stadie, Nicholas P.

Stahl, Frank

Stalikas, Constantine

Stamatatos, Theocharis

Stambuk, Nikola

Stan, Miruna

Stana, Anca-Daniela

Stanciu, Gabriela-Dumitrita

Stancu, Camelia Sorina

Standal, Inger B.

Stanford, Fatima Cody

Stanicová, Jana

Staniek, Halina

Stanimirova, Ivana

Stanisz, Beata 
Stankevic, Marek

Stankova, Ivanka

Stanković, Dalibor

Stankovic, Milan

Stanovnik, Branko

Stare, Jernej

Starecki, Tomasz

Starek, Małgorzata

Štarha, Pavel

Stark, Amy L.

Stark, Lesley A.

Starowicz, Małgorzata

Starykevich, Maksim

Starynowicz, Przemyslaw

Starzak, Karolina

Starz-Gaiano, Michelle

Stasiak-Różańska, Lidia

Staszak, Katarzyna

Staszczak, Magdalena

Statt, Antonia

Statti, Giacarlo

Stavber, Stojan

Stavrianidi, Andrey

Stawiński, Jacek

Stawny, Maciej

Steenari, Britt-Marie

Steenekamp, Jan

Stefanelli, Manuela

Stefania, Filosa

Štefanić, Petra Peharec

Stefanidou, Maria E.

Stefanowicz-Hajduk, Justyna

Stefanska, Anna

Stefanucci, Azzurra

Steger, Gerhard

Steger, Gertrud

Steinbach-Rankins, Jill

Steinbeck, Christoph

Steindel, Mário

Steinhauer, Stephan

Steinhoff, Uwe

Steinmann, Stephan N.

Stellato, Francesco

Stellavato, Antonietta

Stelmach-Mardas, Marta

Stelmachowski, Paweł

Stepanic, Visnja

Stephen, Michael Rajesh

Stephens, Gary

Stepien, Ewa

Stępień, Marcin
Stepnicka, Petr

Štěpnička, Petr

Stępnik, Maciej

Stępniowska, Anna

Ster, Céline

Stern, Raivo

Sterner, Olov

Stetsyshyn, Yurij

Steuer, Christian

STEVANOVIC, Jevrosima

Stewart, James

Stewart, Jason

Stine, Keith J.

Stiriba, Salah-Eddine

Stirke, Arunas

Stobiecka, Magdalena

Stobiecki, Maciej

Stocchero, Matteo

Stoch, Paweł

Stock, Naomi L.

Stocks, Michael

Stoddard, Shana

Stodulski, Maciej

Stogniy, Marina Yu.

Stoica, Anicuta

Stoikov, Ivan Ivanovich

Stojakowska, Anna

Stojanoff, Vivian

Stojanović, Gordana S.

Stojko, Jerzy

Stokke, Bjørn Torger

Stokowa-Sołtys, Kamila

Stolarczyk, Agnieszka

Stolz, Andreas

Stornaiuolo, Mariano

Storsberg, Joachim

Stove, Christophe P.

Stoyanova, Albena

Straatsma, Tjerk

Štrac, Dubravka Švob

Straniero, Valentina

Strankowska, Justyna

Strankowski, Michal

Strappe, Padraig

Strati, Irini F.

Stratikos, Efstratios

Straus, Suzana K.

Strauss, Johannes

Strauss, Laura

Strehmel, Veronika

Strelec, Ivica 
Strianese, Maria

Stripp, Sven T.

Stroffekova, Katarina

Strojny-Cieslak, Barbara

Stroupe, M. Elizabeth

Strout, Douglas L.

Strozzi, Matteo

Strube, Jochen

Struga, Marta

Štrukil, Vjekoslav

Strungaru, Stefan-Adrian

Struszczyk, Marcin Henryk

Stryczewska, Henryka Danuta

Strzelec, Krzysztof

Strzemiecka, Beata

Strzemski, Maciej

Stuani, Lucille

Stuart-Williams, Hilary

Stuchlik, Stanislav

Studzinska-Sroka, Elzbieta

Sturtzel, Caterina

Stürup, Stefan

Stushnoff, Cecil

Stutz, Bernardo

Stylianakis, Minas

Stylianakis, Minas M.

$\mathrm{Su}$, Chie-Shaan

$\mathrm{Su}$, Dan

$\mathrm{Su}$, Hongmei

$\mathrm{Su}$, Peifeng

Su, Weiheng

$\mathrm{Su}$, Wen-Ta

$\mathrm{Su}$, Yichi

$\mathrm{Su}$, Zheng-Yuan

Suarez-Alcantara, Karina

Suaud, Nicolas

Subach, Fedor V.

Šubarić, Drago

Subczynski, Witold K.

Subirats, Xavier

Subramanian, Chitra

Subramanian, Palaniappan

Subramanian, Parthasarathi

Succi, Mariantonietta

Suchanicz, Jan

Suckling, Colin J.

Sud'ina, Galina F.

Suddala, Krishna Chaitanya

Sudo, Atsushi

Suea-Ngam, Akkapol

Sugahara, Takuya
Sugai, Takeshi

Sugamata, Koh

Sugawara, Akihiro

Sugawara-Narutaki, Ayae

Sugier, Danuta

Sugier, Piotr

Sugimoto, Masahiro

Sugimura, Kazuki

Suh, Hyung Joo

Suh, Joonhyuk

Suharoschi, Ramona

Sujin, Hoshi

Sukhikh, Taisiya

Sukocheva, Olga

Sulaeva, Irina

Sulek, Anna

Suleria, Hafiz Ansar Rasul

Suliburska, Joanna

Sulka, Grzegorz Dariusz

Sułkowska-Ziaja, Katarzyna

Sulonen, Mira

Sülsen, Valeria

Sulyok, Michael

Sumby, Christopher

Sumczynski, Daniela

Summo, Carmine

Sun, Caijun

Sun, Chengwen

Sun, Conroy

Sun, Di

Sun, Duanping

Sun, Guohui

Sun, I-Wen

Sun, Jing Zhi

Sun, Lvhui

Sun, Qing-Fu

Sun, Shaolong

Sun, Xiaodan

Sun, Ying

Sun, Yun-Lu

Sun, Zhiwei

Sunatsuki, Yukinari

Sundaram, Paul

Sundararajan, Raji

Sundarrajan, Subramanian

Sundholm, Dage

Sundman, Ola

Sung, Ping-Jyun

Sung, Wen-Chieh

Sungthong, Rungroch

Suntharalingam, Kogularamanan 
Suntornnond, Ratima

Supuran, Claudiu

Surguchov, Andrei

Surleva, Andriana

Surmacki, Jakub Maciej

Suryanarayana, Phanish

Sut, Stefania

Sutherland, Hamish S.

Sutton, Adam T.

Suzuka, Toshimasa

Suzuki, Hideo

Suzuki, Maiko

Suzuki, Ryuichiro

Suzuki, Toshikazu

Suzuki, Toyofumi

Suzutani, Tatsuo

Suzzi, Giovanna

Svavarsson, Halldor Gudfinnur

Svete, Jurij

Svetlana, Tretsiakova-McNally

Svetlov, Maksim

Svoboda, Ladislav

Svoronos, Paris D. N.

Swairjo, Manal A.

Swarbrick, Crystall

Swartz, Talia H.

Swatko-Ossor, Marta

Sweeney, Elizabeth

Świątek, Łukasz

Świątek, Piotr

Świderek, Katarzyna

Swidergall, Marc

Swiecilo, Agata

Świergosz, Tomasz

Świetlicka, Izabela

Świetlik, Roman

Świętnicki, Wiesław

Swiezewska, Ewa

Swift, Thomas

Swinarew, Andrzej

Sychrovsky, Vladimir

Sydnes, Leiv K.

Syguda, Anna

Sykłowska-Baranek, Katarzyna

Sykora, David

Sýkora, Jan

Sylla, Maité

Symianakis, Emmanouil

Syrovets, Tatiana

Sys, Milan

Sytar, Oksana
Sytykiewicz, Hubert

Szabelski, Paweł

Szabo, Andras

Szabó, László

Szabo, Milan

Szacon, Elzbieta

Szafert, Slawomir

Szafranek-Nakonieczna, Anna

Szafraniec-Szczęsny, Joanna

Szafrańska, Katarzyna

Szakiel, Anna

Szakonyi, Zsolt

Szala, Mateusz

Szaleniec, Maciej

Szállási, Árpád

Szałowski, Karol

Szanto, Magdolna

Szatmári, Tünde

Szczepański, Piotr

Szczubialka, Krzysztof

Szebenyi, Marian

Széchenyi, Aleksandar

Szécsi, Mihály

Szeiffova Bacova, Barbara

Szekely, Gyorgy

Szeleszczuk, Łukasz

Szendrő, Katalin

Szeto, Savio Yim Tong

Szewczyk, Agnieszka

Szewczyk, Katarzyna

Szewczyk, Roman

Szewczyk-Golec, Karolina

Szidarovszky, Tamás

Szilvási, Tibor

Szlachcic, Anna

Szlęk, Jakub

Szoboszlai, Norbert

Szoka, Łukasz

Szöllösi, György

Szolnoky, Gyozo

Szoor, Arpad

Szopa, Agnieszka

Szopka, Katarzyna

Szostak, Michal

Szostak, Roman

Szpyrka, Ewa

Sztanke, Krzysztof

Sztanke, Małgorzata

Szubert, Karol

Szukalski, Adam

Szulc, Karolina 
Szulczyk, Daniel

Szultka-Młyńska, Małgorzata

Szumny, Antoni

Szumski, Michal

Szwajca, Anna

Szweda, Piotr

Szwengiel, Artur

Szyja, Bartłomiej M.

Szymandera-Buszka, Krystyna

Szymańki, Paweł

Szymanska-Chargot, Monika

Szymborski, Tomasz

Ta, Hang

Tabacaru, Aurel

Tabanca, Nurhayat

Tabassum, Shawana

Tabata, Yasuhiko

Tabatabai, Habib

Tabernero, Vanessa

Tabernero-Duque, María-Jesús

Tada, Kohei

Taddei, Marco

Taddei, Maurizio

Tadić, Vanja

Tadyszak, Krzysztof

Taffa, Dereje Hailu

Tafuri, Simona

Taga, Yuki

Tagad, Harichandra

Tagad, Harichandra D.

Tagit, Oya

Tagliatesta, Pietro

Tagliazucchi, Davide

Taglienti, Anna

Taglietti, Angelo Maria

Tago, Kenji

Taguchi, Toru

Taha, Muhammad

Tahara, Hironobu

Tai, Dar-Fu

Taira, Junsei

Tait, Steven L.

Tajti, Ádám

Takada, Akihiko

Takafuji, Makoto

Takahashi, Fumiki

Takahashi, Kaito

Takahashi, Kazuo

Takahashi, Ken

Takahashi, Masaki

Takahashi, Nobuaki
Takahashi, Osamu

Takai, Chika

Takanami, Toshikatsu

Takase, Hideki

Takashima, Eizo

Takasu, Kiyosei

Takatori, Kazuhiko

Takaya, Tomohisa

Takayoshi, Suzuki

Takayuki, Tsukui

Takeda, Youhei

Takegahara, Noriko

Takenaga, Mitsuko

Takenaka, Shigeori

Takeoka, Shinji

Takeshita, Fumitaka

Takeuchi, Hideyuki

Takizawa, Shinobu

Talhi, Oualid

Taliani, Sabrina

Talimian, Ali

Tallawi, Marwa

Talora, Claudio

Taly, Antoine

Tam, Eric Kwok Wai

Tam, Roger Y.

Tamaian, Radu

Tamainot-Telto, Zacharie

Tambe, Mitali

Tambuwala, Murtaza

Tampa, Mircea

Tampieri, Francesco

Tamulevičienè, Asta

Tamura, Atsushi

Tamura, Osamu

Tan, Angel

Tan, Bee K.

Tan, Bee Kang

Tan, Chen

Tan, Chengxia

Tan, Joanne T.M.

Tan, Lik Tong

Tan, Ming-Qian

Tan, Swee Ching

Tan, Yanglan

Tan, Yaw Sing

Tan, Ying

Tan, Zhijian

Tanabe, Makoto

Tanabe, Shihori

Tanabe, Yoo 


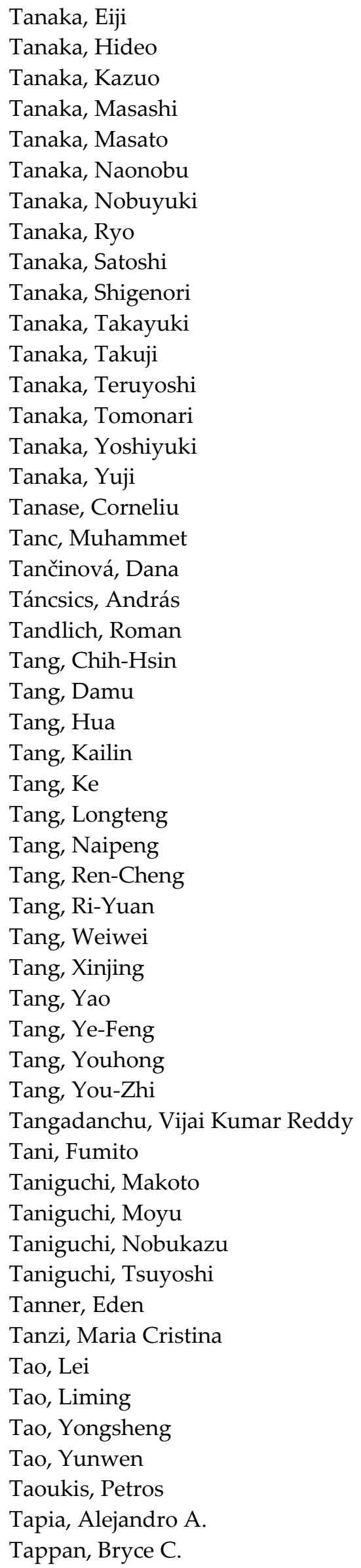

Tanaka, Eij

Tanaka, Hideo

Tanaka, Kazuo

Tanaka, Masashi

Tanaka, Masato

Tanaka, Naonobu

Tanaka, Nobuyuk

Tanaka, Ryo

Tanaka, Satoshi

Tanaka, Shigenori

Tanaka, Takayuki

Tanaka, Yoshiyuki

Tanaka, Yuji

Tanase, Corneliu

Tanc, Muhammet

Tančinová, Dana

Táncsics, András

Tandlich, Roman

Tang, Chih-Hsin

Tang, Damu

Tang, Hua

Tang, Kailin

Tang, Ke

Tang, Longteng

Tang, Naipeng

Tang, Ren-Cheng

Tang, Xinjing

Tang, Yao

Tang, Ye-Feng

Tang, Youhong

Tang, You-Zhi

Tangadanchu, Vijai Kumar Reddy

Tani, Fumito

Taniguchi, Makoto

Taniguchi, Moyu

Taniguchi, Nobukazu

Taniguchi, Tsuyoshi

Tanner, Eden

Tanzi, Maria Cristina

Tao, Lei

Tao, Liming

Tao, Yongsheng

Tao, Yunwen

Tapia, Alejandro A.

Tappan, Bryce C.
Tappia, Paramjit

Tarabanko, Nikolay

Tarabanko, Valery E.

Tarallo, Oreste

Taranto, Alex Gutterres

Tarasiuk, Jolanta

Tarasov, Andrei

Tard, Cédric

Tarkowská, Danuše

Tarkowski, Petr

Tarozzi, Andrea

Tarrago, Lionel

Tartaglia, Angela

Tashima, Alexandre K.

Tashima, Toshihiko

Tashiro, Mitsuru

Tasic, Ljubica

Tasinato, Nicola

Tasso, Bruno

Tata, Rama Rao

Tateishi, Kensuke

Tatikolov, Aleksander S.

Tatsuhiro, Kojima

Tatsuya, Kato

Tatullo, Marco

Taurin, Sebastien

Tava, Aldo

Tavakoli, Javad

Tavani, Cinzia

Tavanti, Francesco

Tavares, José Carlos

Tavares, Josean Fechine

Tavladoraki, Paraskevi

Tawfike, Ahmed

Tayebati, Seyed Khosrow

Tayel, Amr

Taylor, Erika A.

Tchertanov, Luba

Tchetina, Elena V.

Tchoukov, Plamen

Tecilla, Paolo

Tecpoyotl-Torres, Margarita

Tedeschi, Paola

Tedesco, Idolo

Teghil, Roberto

Tegl, Gregor

Teif, Vladimir

Teixeira, Alfredo

Teixeira, Hellen S.

Teixeira, João M. C.

Teixeira, Luís Filipe 
Teixeira, Natércia

Teixeira, Rita

Teixeira, Róbson

Teixeira-Santos, Rita

Teixidó, Jordi

Tejero, Jesús

Tekko, Ismaiel

Telang, Sucheta

Téletchéa, Stéphane

Tellez Jurado, Lucia

Téllez-Téllez, Maura

Téllez-Valencia, Alfredo

Temesvari, Lesly

Temussi, Piero A.

Teng, Ba-Bie

Teng, Peng

Tenhaeff, Wyatt

Teo, Jeremy

Teobald, Kupka

Teodoro, João

Tera, Masayuki

Teramoto, Hidetoshi

Teramoto, Naozumi

Teramoto, Yoshikuni

Terec, Anamaria

Terent'ev, Alexander O.

Terentjeva, Margarita

Terenzi, Alessio

Terhorst, Cox

Termolino, Pasquale

Termopoli, Veronica

Ternesten-Hasséus, Ewa

Terpos, Evangelos

Terpou, Antonia

Terraneo, Giancarlo

Terrasson, Vincent

Terrinoni, Alessandro

Teschke, Rolf

Tesic, Zivoslav

Teslic, Nemanja

Tetsuo, Okada

Teusch, Nicole

Tews, Daniel

Texido Bartes, Robert

Tezuka, Yasuhiro

Thakur, Ashutosh

Thakur, Sachin

Thakur, Vijay

Thakur, Vijay Kumar

Thalhammer, Gregor

Thangaraj, Annadurai
Thapa, Manoj

Thapa, Surendra

Theodorakis, Emmanuel A.

Theodossiou, Theodossis A.

Theoduloz, Cristina

Thi, Thai Thanh Hoang

Thiebault, Thomas

Thiel, Gerald

Thies, Stephan

Thiriet-Rupert, Stanislas

Thives, Liseane Padilha

Thomas, Ananya

Thomas, Eric Jim

Thomas, John

Thomas, Maciej

Thomas, Morgan L.

Thomas, Robert K.

Thomas, Stephen P.

Thomas, Thresia

Thomas, Tiffany

Thomas, Wayne

Thompson, Miles D.

Thompson, Roney

Thompson, Stephen

Thomson, Jennifer

Thorpe, Clare

Thybring, Emil Engelund

Tiago, Gonçalo

Tian, Chun-Jie

Tian, Furong

Tian, Guocai

Tian, Jiangwei

Tian, Kun Viviana

Tian, Li

Tian, Shung Wu

Tian, Weiguo

Tian, Ye

Tiano, Luca

Tibullo, Daniele

Tidgewell, Kevin

Tietze, Daniel

Tikhonov, Vladimir

Tikhonova, Irina

Timoshenko, Alexander V.

Timson, David J.

Ting, Richard C. H.

Tintaru, Aura

Tipeev, Azat

Tiperciuc, Brindusa

Tirado, Diego F.

Tircso, Gyula 
Tirillini, Bruno

Tiritan, Maria Elizabeth

Tiriveedhi, Venkataswarup

Tischler, Dirk

Tisovský, Pavol

Titov, Aleksei A.

Tiwari, Rakesh K.

Tiyyagura, Hanuma Reddy Tjandra, Nico

Tkac, Jan

Tlili, Anis

To, Ching Tat

Tobiszewski, Marek

Tobrman, Tomáš

Tocmo, Restituto

Todasca, Maria-Cristina

Todde, Sergio

Todorović, Vanja M.

Toepel, Jörg

Tofana, Maria

Tofani, Daniela

Toffolo, Michael

Toillon, Robert-Alain

Toiu, Anca

Tojo, Tomohiro

Tokarz, Barbara

Tokarz-Sobieraj, Renata

Tokmina-Lukaszewska, Monika

Toledo-Neira, Carla

Tolga, Karsili

Tolgyessy, Peter

Tolkou, Athanasia

Tolomelli, Alessandra

Tolosa, Ezequiel

Toma, Lucio

Tomai, Pierpaolo

Tomanek, Boguslaw

Tomás-Barberán, Francisco A.

Tomasello, Barbara

Tomasevic, Igor

Tomasik, Piotr

Tomasini, Claudia

Tomášková, Jana

Tomaszewska, Ewa

Tomaz, Ivana

Tombelli, Sara

Tomczyk, Wojciech

Tomé, Augusto C.

Tomek, Petr

Tomer, Vijay K.

Tomi, Félix
Tomić, Simonida

Tomoda, Hiroshi

Tomonaga, Shozo

Tomuta, Ioan

Tonazzi, Annamaria

Tondi, Donatella

Tonelli, Michele

Tong, Shengqiang

Tong, Wenming

Tong, Yexiang

Tönges, Lars

Tonolo, Giancarlo

Tonutti, Pietro

Topoglidis, Emmanuel

Toprakci, Hatice Aylin Karahan

Topuz, Fuat

Toriba, Taiyo

Toribio, Laura

Torigoe, Kanjiro

Torikai, Kohei

Tormo, Jose

Torneiro, Mercedes

Tornesello, Anna Lucia

Toro, Ayelén

Toro, Roberta G.

Torok, Marianna

Toropov, Andrey

Torović, Ljilja

Torre, Maria Luisa

Torre, Renato

Torregiani, Elisabetta

Torres Hernández, Yadir

Torres Perez, María Dolores

Torres, Adrian Gabriel

Torres, Sofia

Torres-Giner, Sergio

Torres-Padrón, María Esther

Torres-Ramírez, Eduardo

Torri, Luisa

Torricelli, Paola

Torrico, Damir D.

Torroba, Tomas

Torzewska, Agnieszka

Tosa, Monica Ioana

Tosha, Takehiko

Tosheva, Lubomira

Toshio, Koizumi

Tošner, Zdeněk

Tothova, Csilla

Toti, Kiran

Totsingan, Filbert 
Totti, Federico

Tõugu, Vello

Touraud, Didier

Tournié, Aurélie

Toušek, Jaromír

Toyama, Ikuo

Toyoda, Yu

Toyohara, Jun

Tőzsér, József

Träbert, Elmar

Trabocchi, Andrea

Traboni, Serena

Trader, Darci

Trafiałek, Joanna

Trajkovic, Vladimir

Trammell, Samuel

Trammell, Scott A.

Tran, Trong

Trandafirescu, Cristina-Maria

Trani, Antonio

Tranmer, Geoff

Trapani, Adriana

Trapella, Claudio

Tratnik, Janja Snoj

Travlos, Ilias

Trcek, Janja

Trdan, Stanislav

Treebak, Jonas

Tremonte, Patrizio

Trequattrini, Francesco

Tres, Marcus Vinicius

Tretyakov, Evgeny V.

Trevaskis, Natalie

Trevisan, Andrea

Triantafyllidis, Konstantinos

Tribello, Gareth

Tricot, Gregory

Trifonov, Rostislav

Trifonova, Oxana P.

Trigger, Sergey

Trilleras, Jorge

Trimigno, Alessia

Trincone, Antonio

Trindle, Carl

Trinh, Thuat

Triola, Gemma

Tripathi, Jaindra Nath

Tripathi, Manisha

Tripodi, Gianluca

Tripodo, Giuseppe

Trippier, Paul C.
Tritschler, Laurent

Trivanovic, Drenka

Trofimova, Maya

Trögl, Josef

Trognitz, Friederike

Trojanowicz, Marek

Trombini, Claudio

Troncoso, Ana María

Tropea, Alessia

Troppmair, Jakob

Trotsko, Nazar

Trovato, Fabio

Trubetskaya, Anna

Trucillo, Paolo

Trujillo, Antonio-José

Trujillo, Cristina

Trujillo-Cayado, Luis

Trujillo-Rodríguez, María J.

Trujillo-Rodríguez, María José

Trumble, John T.

Truong, Vi-Khanh

Trushkov, Igor V.

Trusso Sfrazzetto, Giuseppe

Trusso, Sebastiano

Trybuła, Zbigniew

Trzaskowski, Bartosz

Trzcińska, Monika

Trzeciak, Anna

Tsai, Chen-Yen

Tsai, Hui-Lien

Tsai, Jen-Chieh

Tsai, Pi-Jen

Tsai, Shu-Huai

Tsai, Shu-Yao

Tsai, Wan-Yu

Tsakalof, Andreas

Tsakovska, Ivanka

Tsaltas, Dimitris

Tsang, Michael

Tsantili-Kakoulidou, Anna

Tsarkova, Larisa

Tsatsakis, Aristidis

Tsatsanis, Christos

Tscheliessing, Rupert

Tse, William K.F.

Tse-Dinh, Yuk-Ching

Tseng, Chih-Hua

Tseng, Wei-Lung

Tsentalovich, Yuri P.

Tserepi, Angeliki

Tsiafoulis, Constantinos G. 
Tsibouklis, John

Tsimidou, Maria

Tsimpanogiannis, Ioannis N.

Tsirelson, Vladimir G.

Tsirka, Kyriaki

Tsironi, Theofania

Tsitsilianis, Constantinos

Tsitsilonis, Ourania E.

Tsivileva, Olga

Tskhovrebov, Alexander G.

Tsopelas, Fotis

Tsopmo, Apollinaire

Tsotsalas, Manuel

Tsoupras, Alexandros

Tsubaki, Kazunori

Tsuchida, Sachio

Tsuchiya, Hiroyuki

Tsuji, Atsushi B.

Tsuji, Hideto

Tsukamoto, Takashi

Tsumuraya, Takeshi

Tsuneda, Takao

Tsunedomi, Ryouichi

Tsunoda, Makoto

Tsurkan, Mikhail V.

Tsutsumi, Hiroshi

Tsutsumi, Osamu

Tsvetkov, Nikolai

Tsyganov, Viktor E.

$\mathrm{Tu}$, Jun-Ling

Tu, Min

$\mathrm{Tu}$, Zhengkai

Tubaro, Cristina

Tubaro, Franco

Tubić, Aleksandra

Tuccinardi, Tiziano

Tuci, Giulia

Tucker, Thomas J.

Tudoroiu, Nicolae

Tufa, Ramato Ashu

Tufariello, Maria

Tufariello, Miriam

Tullio, Vivian

Tullius Scotti, Marcus

Tůma, Karel

Tůma, Zdeněk

Tumova, Lenka

Tun, Mya Myat Ngwe

Tundis, Rosa

Tunesi, Marta

Tungmunnithum, Duangjai
Tunick, Michael

Tunon, Inaki

Tuovinen, Olli

Turanek, Jaroslav

Turano, Paola

Turck, Christoph W.

Turco, Antonio

Turco, Rosa

Turhanen, Petri

Turnaturi, Rita

Turnbull, Matthew W.

Turner, Alice M.

Turner, David

Turowski, Tomasz W.

Turrini, Eleonora

Turrini, Federica

Turunen, Lotta

Tutone, Marco

Tuzimski, Tomasz

Tvrdeic, Ante

Twarużek, Magdalena

Tyler, Robert

Tylewicz, Urszula

Tyliszczak, Bożena

Tylkowski, Bartosz

Tymecki, Łukasz

Tyski, Stefan

Tyystjärvi, Esa

Tzanavaras, Paraskevas D.

Tzeli, Demeter

Tzen, Jason T.

Tzeng, Biing-Chiau

Tzeng, Shiang-Jong

Tzeng, Shu-Ling

Tzin, Vered

Tziveleka, Leto-Aikaterini

Tzortzakis, Nikos

Tzovenis, Ioannis

Tzvetkova, Pavleta

Uan, Jun-Yen

Ubeda, Cristina

Ubiali, Daniela

Uchida, Satoshi

Uchida, Yoshiaki

Uchihashi, Takayuki

Uchikoshi, Masahito

Uchiyama, Taketo

Udal'tsov, Alexander

Uddin, Jamal

Udrescu, Lucretia

Uehara, Tomoya 
Uekusa, Hidehiro

Ueno, Mikinori

Uetani, Kojiro

Uetrecht, Charlotte

Uetz, Peter

Ufnal, Marcin

Uivarosi, Valentina

Ukalska-Jaruga, Aleksandra

Ullah, Irfan

Umehara, Mikihisa

Umerska, Anita

Ungurianu, Anca

Unković, Nikola

Unno, Keiko

Uno, Tomohide

Unsworth, Will

Updegrove, Taylor B.

Urakawa, Osamu

Urban, Milan

Urban, Philippe

Urbańczyk-Lipkowska, Zofia

Urbanek, Pavel

Urbaniak, Alicja

Urra, Félix A.

Urriolabeitia, Esteban

Urso, Elena

Urzi, Clara

Usai, Donatella

Usai, Marianna

Usama, Syed Muhammad

Usami, Yoshihide

Ushijima, Kentaro

Ussowicz, Marek

Usui, Kenji

Usuki, Toyonobu

Usuki, Yoshinosuke

Utikar, Ranjeet P.

Uusi-Oukari, Mikko

Uversky, Vladimir

Uziel, Orit

V. Likhanova, Natalya

Vaculovičová, Marketa

Vafidis, Dimitris

Vaghasiya, Jayraj

Vagin, Mikhail

Vago, Riccardo

Vahabi, Henri

Vaidyanathan, Ganesan

Vaidyanathan, Sriram

Vakh, Christina S.
Vakros, John

Valandro, Silvano

Valcarce, David G.

Valdersnes, Stig

Vale, Carlos Alberto Garcia Do

Vale, Paulo

Valentao, Patricia

Valente, Artur J. M.

Valente, Joana

Valente, Ligia

Valentova, Jindra

Valentová, Kateřina

Valenzuela, Miguel

Valenzuela, Rodrigo

Valero-Cases, Estefanía

Valgimigli, Luca

Valiante, Vito

Valitutti, Francesco

Valiveti, Chaitanya

Valkenier, Hennie

Valle, Luisa Dalla

Vallée, Yannick

Vallejo, Javier P.

Valles, Soraya L.

Valles, Vincent

Valletta, Alessio

Valsami, Georgia

Valyaev, Dmitry A.

Vamanu, Emanuel

Van Aerschot, Arthur

Van Bennekom, W.P.

Van Beusechem, Victor

Van Bommel, Maarten R

Van Deenen, Nicole

Van Den Berg, Marco Alexander

Van Den Ende, Wim

Van Den Meiracker, Anton H.

Van Der Kamp, Jan Willem

Van Der Meer, Marcel T. J.

Van Der Merwe, Marie

Van Der Vlugt, Jarl Ivar

van Duijn, Bert

Van Dyke, Michael

Van Hecke, Kristof

Van Herwijnen, Hendrikus

Van Hoecke, Karen

Van Horn, Wade

Van Lis, Robert

Van Mourik, Tanja

Van Noorden, Cornelis Johannes

Forrindinis 
Van Ree, Teunis

Van Rijn, Richard M.

Van Steenberge, Paul Hm M.

Van Zyl, Werner

Van, Thi Thu Hao

Vance, David

Vande Velde, Christophe

Vanden Eynde, Jean Jacques

Vandenbroeck, Koen

Vanderlei, Maria De Fátima

Vandooren, Jennifer

Vanella, Luca

Vanin, Fernanda Maria

Vannacci, Alfredo

Vanni, Ester

Vannini, Andrea

Vanthuyne, Nicolas

Ványolós, Attila

Vapnik, Yevgeny

Varadarajan, Shankar

Váradi, Judit

Varani, Luca

Varchi, Greta

Vardhan, Harsh

Varga, Gábor

Varga, Szabolcs

Varga, Zoltan

Vargas Jentzsch, Paul

Varghese Gupta, Sheeba

Varma, Rajender S.

Varna, Mariana

Várnagy, Katalin

Varoni, Elena Maria

Varotto, Serena

Varvounis, George

Vasas, Andrea

Vasco Vidal, Aldrin

Vasco-Calle, Diego Andrés

Vascotto, Carlo

Vasdev, Neil

Vasic, Vesna

Vasicek, Ondrej

Vasile, Bogdan Stefan

Vasile, Francesca

Vasilescu, Alina

Vasiliev, Mikhail M.

Vasilyev, Aleksander V.

Vašková, Janka

Vasos, Paul

Vasquez, Marlen
Vassallo, Antonio

Vassilev, Nikolay G.

Vassilis J, Inglezakis

Vasu, Dhananjayan

Vatsadze, Sergey

Vaughan, Roger

Vaughey, John

Vavitsas, Konstantinos

Vayá Pérez, Ignacio

Vaz Junior, Silvio

Vaz, Daniela C.

Vaz, Josiana A.

Vaz, Pedro D.

Vazquez Belda, Beatriz

Vázquez Duhalt, Rafael

Vazquez Espinosa, Mercedes

Vazquez, Elba S.

Vázquez-Ovando, Alfredo

Vázquez-Rodríguez, Gabriela A.

Vázquez-Tato, M. Pilar

Vaz-Velho, Manuela

Vecchio Ciprioti, Stefano

Vedernikov, Andrei N.

Védrine, Jacques $\mathrm{C}$.

Veenman, Leo

Vega Holm, Margarita

Vegvari, Akos

Vehus, Tore Sandnes

Veit, Guido

Vejpravova, Jana

Vejux, Anne

Vek, Viljem

Vekariya, Rakesh

Velasco, Rebeca Hernandez

Velasco-Torrijos, Trinidad

Velena, Astrida

Velgosova, Oksana

Velikova, Tsvetelina

Vella, Filomena Monica

Vellecco, Valentina

Velmuzhov, Alexander

Veltri, Lucia

Velu, Sadanandan E.

Vemulapalli, Vidyasiri

Vendamme, Richard

Vendrell, Josep

Venerando, Andrea

Venkatashamy Reddy, Mogalahalli

Venkatesh

Venneri, Francesca

Vennerstrom, Jonathan 
Venskutonis, Petras Rimantas

Ventrella, Domenico

Ventruti, Gennaro

Ventura, Cinzia Anna

Venugopal, Dhamodharan

Venuti, Elisabetta

Verbanac, Donatella

Verde, Ignacio

Verdejo, Begoña

Verdú-Andrés, Jorge

Veremeichik, Galina N.

Veres, Miklos

Verestiuc, Liliana

Verga, Daniela

Vergara, Daniele

Vergara, Fredd

Vergara, José

Verger, Stéphane

Verho, Oscar

Verhoeven, Adrie

Verhoog, Stefan

Veríssimo, Marta

Verly, Rodrigo Moreira

Vermathen, Martina

Vermeulen, Marc

Vernardou, Dimitra

Verona, Guglielmo

Veronesi, Federico

Verotta, Luisella

Verri, Tiziano

Verri, Waldiceu A.

Vervandier-Fasseur, Dominique

Verza, Simone Gasparin

Vesa, Stefan Cristian

Vesey, David

Veskoukis, Aristidis S.

Vessieres, Anne

Vetvicka, Vaclav

Veverka, Vaclav

Veyron-Churlet, Romain

Vianello, Robert

Viapiana, Agnieszka

Viappiani, Cristiano

Vicario, Javier

Vicaș, Laura Grațiela

Vicente Crespo, Gemma

Vicente, Cristina P.

Vicente, Eduardo Festozo

Vicente, Francisca

Vicente-Manzanares, Miguel

Vícha, Robert
Victor J., Rico

Victor, Bruno

Vidal, Fernando

Videau, Patrick

Vidic, Jasmina

Vidovic, Bojana

Vieillard, Julien

Viejo, Claudia Gonzalez

Vielba, Jesús María

Viennet, Thibault

Vierra, Craig

Vigier, Karine

Víglaský, Viktor

Viglašová, Eva

Vigneresse, Jean Louis

Vijayakumar, Sarath

Vijayan, Madhuvanthi

Vijgenboom, Erik

Viktorova, Jitka

Vikulina, Anna

Vil', Vera A.

Vila, Carlos

Vila, Fernando D.

Viladomat, Francesc

Vilaivan, Tirayut

Vilanova, Xavier

Vilas, Jose Luis

Vilela, Alice

Vilgis, Thomas

Villa, Carla

Villa, Chiara

Villa, Cristian

Villa, Stefania

Villa-Morales, María

Villani, Claudio

Villar, Marcelo

Villarreal, Vicent Sixte Safont

Villarreal-Gómez, Luis Jesús

Villaseñor, Alma

Villaverde, Juan José

Villegas, Victoria

Villena, Juan

Villorbina, Gemma

Viña, Dolores

Viña, Jaime

Vinarov, Zahari

Viñas, Clara

Viñas, Miguel

Vinauger, Clement

Vincent, John B.

Vincent-Bonnieu, Sebastien 
Vincenzi, Colombina

Vincenzi, Fabrizio

Vincenzo, De Leo

Vincze, Eva

Vinet, Raúl

Vinnik, Denis

Vinodh, Rajangam

Vinšová, Jarmila

Vinueza, Nelson

Viola, Giampietro

Viola, Manuela

Viola-Villegas, Nerissa Therese

Vione, Davide

Virag, Laszlo

Virgili, Fabio

Virgili, Tersilla

Virjamo, Virpi

Virto, Mailo

Visconti, Roberta

Visentin, Andrea

Visentin, Fabiano

Visentin, Silvia

Vishe, Mahesh

Viškelis, Jonas

Viškelis, Pranas

Viskup, Richard

Vismara, Elena

Viso Beronda, Alma

Vistoli, Giulio

Vitale, Fabrizio

Vitale, Luca

Vitalini, Sara

Vitasović Kosić, Ivana

Viter, Roman

Vitetta, Luis

Viva, Federico A.

Vives, Guillaume

Vivet, Alexandre

Vizireanu, Camelia

Vlachou, Marilena

Vladescu, Marian

Vladić, Jelena

Vladimirov, Vladimir

Vlainić, Josipa

Vlase, Gabriela

Vlasiou, Manos

Voća, Neven

Vodeničarovová, Melita

Vodyankina, Olga

Vögele, Martin

Vogt, Sarah
Vokurka, Aleš

Volcho, Konstantin

Voliani, Valerio

Volkova, Elena G.

Volmajer, Julija

Volodin, Alexander M.

Vomhof-DeKrey, Emilie

Von Eggeling, Ferdinand

Von Knethen, Andreas

Vora, Ankit

Vorobyeva, Anzhelika

Vorontsov, Alexander

Vorsa, Nicholi

Vosloo, Hermannus C. M.

Vostinaru, Oliviu

Vostrikova, Kira E.

Voutsinas, Gerassimos E.

Voyiatzis, George

Vozzi, Giovanni

Vrabel, Milan

Vračko, Marjan

Vranes, Milan

Vranic, Edina

Vriend, Jerry

Vrinceanu, Narcisa

Vrontaki, Eleni

Vukojevic, Katarina

Vukomanovič, Mariija

Vulic, Jelena J.

Vuorimaa-Laukkanen, Elina

Vuthaluru, Hari Babu

Vyas, Vijay

Vynios, Demitrios

Vyšniauskas, Aurimas

Vyviurska, Olga

Waagbo, Rune

Wacher, María Del Carmen

Waczulíková, Iveta

Wagner, Anika

Wagner, Pawel

Wahli, Walter

Wajs-Bonikowska, Anna

Wakao, Masahiro

Wakioka, Masayuki

Waksmundzka-Hajnos, Monika

Walach, Wojciech

Wałajtys-Rode, Elżbieta

Walczak, Krzysztof

Walczyński, Krzysztof

Waldum, Helge

Wałecka-Zacharska, Ewa 
Wałęsa-Chorab, Monika

Walker, Greg

Walker, Heather

Walker, Louise A.

Wall Medrano, Abraham

Wallace, David R.

Wallace, Gregory Q.

Wallace, Heather M.

Wallace, Karl

Wallace, M. Ariel

Walsby, Charles

Walsh, Kerry

Walter, Jeske

Walton, John

Walwyn, Wendy M.

Walz, Juliane

Wan, Jieping

Wanag, Agnieszka

Wang, Be-Jen

Wang, Bo

Wang, Ceming

Wang, Changning

Wang, Chao-Min

Wang, Chenguang

Wang, Chi Chiu

Wang, Chih-Chieh

Wang, Ching-Chiung

Wang, Chuanlong

Wang, Congzhou

Wang, Danhong

Wang, Di

Wang, Fazuo

Wang, Fenglai

Wang, Fuke

Wang, Fupeng

Wang, Fuyi

Wang, Guangshun

Wang, Guankui

Wang, Guifang

Wang, Guliang

Wang, Hsiuying

Wang, $\mathrm{Hu}$

Wang, Huaishan

Wang, Huanchen

Wang, Hui

Wang, Hui-Chun

Wang, Hui-min

Wang, Huxuan

Wang, Jeffrey

Wang, Jiabin

Wang, Jian
Wang, Jianbo

Wang, Jianhua

Wang, Jin

Wang, Julie Tzu-Wen

Wang, Jun

Wang, Kai

Wang, Kankan

Wang, Li

Wang, Ling

Wang, Liping

Wang, Liqiang

Wang, Lirong

Wang, Lu

Wang, Luyu

Wang, Michael Cai

Wang, Mindy Y.

Wang, Ming-Fu

Wang, Nan

Wang, Nian

Wang, Pei-Ming

Wang, Peng

Wang, Pengcheng

Wang, Pingyuan

Wang, Qingde

Wang, Qingsong

Wang, Qun

Wang, Ruiyong

Wang, San-Lang

Wang, Sheng-Shih

Wang, Shenqi

Wang, Shihua

Wang, Shin-Wei

Wang, Shue

Wang, Shyiwu

Wang, Songlin

Wang, Taoran

Wang, Tengfei

Wang, Tong-Hong

Wang, Tzu-Fan

Wang, Wei

Wang, Wenjing

Wang, Xiaoping

Wang, Xiaoxin

Wang, Xiaoyong

Wang, Xinkun

Wang, Xinwen

Wang, Xizu

Wang, Xu

Wang, Yanbin

Wang, Yeng-Tseng

Wang, Yifei 
Wang, Youxin

Wang, Yu

Wang, Yuesheng

Wang, Yun-Ming

Wang, Zhenxin

Wang, Zhihan

Wang, Zhijun

Wang, Zhongyang

Wang, Zongjie

Ward, John

Ward, Michael D.

Ware, Vassie

Warren, J. David

Warren, Warren S.

Waser, Mario

Wasilewski, Tomasz

Wasylka, Justyna Płotka

Watanabe, Ryuichi

Waterhouse, Andrew

Watkins, A. Neal

Watkins, Gordon Leonard

Watzky, Murielle

Wawrzyniak, Jolanta

Wdowin, Magdalena

Webb, Ian

Webba Da Silva, Mateus

Weber, Birgit

Weber, John

Wedekind, Joseph E.

Weeks, Brandon

Weghuber, Julian

Wegrzyn, Grzegorz

Wei, Cunxu

Wei, Da-Hua

Wei, Gang

Wei, Hua

Wei, Jianjun

Wei, Kongchang

Wei, Wanxing

Wei, Yufeng

Wei, Zhaojun

Wei, Zhongbao

Weidner, Steffen

Weigand, Wolfgang

Weina, Peter J.

Weinberger, Christian

Weis, Martin

Weiss, Richard

Weiss, Siegfried

Welch, Kevin

Welker, Mark
Weller, Michael G.

Wells, Tracy

Wen, Xiaofeng

Wen, Zhiwei

Weng, Ching Feng

Wenta, Tomasz

Wente, Nicole

Wenzel, Barbara

Werlé, Christophe

Wesołowski, Marek

Wessel, Gary M.

West, James D.

Westcott, Stephen A.

Weston, Paul

Westphal, Adrie

Westwell, Andrew

White, Peter

White, Robert L.

White, Rosemary

Whyte, Claire

Wianowska, Dorota

Wibowo, David

Wicker, Louise

Wiczkowski, Wieslaw

Widenmeyer, Marc

Widomska, Justyna

Wieczorek, Marcin

Wieczorek, Piotr

Wieczorek, Piotr P.

Wieczorek, Rafal M.

Wieczorek, Robert

Wiedman, Gregory

Wielgomas, Bartosz

Wiener, Reuven

Wieszczycka, Karolina

Wietrzyk, Joanna

Wietstock, Philip

Wigle, Jeffrey

Wiglusz, Katarzyna

Wiktor, Artur

Wilhelm, Rene

Wilkinson, Jenny

Wilkowska, Agnieszka

Willaert, Ronnie

Willers, Clarissa

Williams, Antony

Williams, Lynda

Williams, Noelle S.

Williams, Vance

Williamson, Vernell

Willmore, William G. 
Wills, Martin

Wilner, Samantha E.

Wilson, Anne M.

Wilson, Danny W.

Wilson, Jeffrey M.

Wilson, Justin J.

Wilson, Peter

Wilson, Philippe B.

Wilton, Steve

Wimmer, Zdenek

Winckler, Thomas

Windholz, Laurentius

Windshügel, Björn

Wink, Michael

Wińska, Katarzyna

Winter, Horst Henning

Wiraja, Christian

Wisniewski, Marek

Wiśniewski, Marek

Witczak, Carol

Witczak, Mariusz

Witczak, Zbigniew J.

Witkowski, Artur

Witt, Katarzyna

Witte, Martin

Włodarczyk, Maciej

Włodarczyk, Zbigniew

Włodarczyk-Stasiak, Marzena

Wnuk, Agnieszka

Wnuk, Maciej

Wnuk, Stanislaw

Woelwer-Rieck, Ursula

Wojaczyńska, Elżbieta

Wójciak, Magdalena

Wojciechowski, Kamil

Wojcieszak, Robert

Wojcik, Cezary

Wojdylo, Aneta

Wojnarowicz, Jacek

Wojtkielewicz, Agnieszka

Wójtowicz, Agnieszka

Wojtunik-Kulesza, Karolina A.

Wojtunik-Kulesza, Karolina Anna

Wołejko, Elżbieta

Wolf, Christoph

Wolf, Katarina

Wolff, Marcus

Wolff, Max

Wölfle, Ute

Wolfson, Adi

Wolny, Juliusz A.
Wołowicz, Anna

Wołowiec, Stanisław

Wolter, Tilman

Won, Kyung Jong

Won, Moo-Ho

Won, Seunggun

Wondrak, Georg

Wong, Alan

Wong, Chung

Wong, Clarence T. T.

Wong, Fung-Fuh

Wong, Maurice

Wong, Wai-Yeung

Wong, Yung Hou

Woo, Sun Hee

Wood, Troy D.

Woscholski, Rudiger

Wöstemeyer, Johannes

Wowalczyk, Wioleta

Wozniak, Jarosław

Woźniak, Łukasz

Woźniak-Budych, Marta

Wozniak-Knopp, Gordana

Wright, Gareth S. A.

Wrobel, Rafał

Wróbel, Tomasz

Wrobel, Tomasz P.

Wróblewska, Agnieszka

Wróblewski, Karol

$\mathrm{Wu}$, Alan

Wu, Bin

$\mathrm{Wu}$, Cen

$\mathrm{Wu}$, Chao

$\mathrm{Wu}$, Chiao-En

Wu, Chia-Wen

Wu, Chieh-Hsi

$\mathrm{Wu}$, Chi-Rei

Wu, Chongming

Wu, Chun-Chi

$\mathrm{Wu}$, Chung-Chun

$\mathrm{Wu}$, Chung-Hsin

$\mathrm{Wu}, \mathrm{Di}$

$\mathrm{Wu}, \mathrm{Fu}-\mathrm{Gen}$

Wu, Hongiing

Wu, Ho-Shing

Wu, Hung-Chin

$\mathrm{Wu}$, Jian-Yong

Wu, Jing-Yun

Wu, Jin-Yi

Wu, Kaiyu

Wu, Kejun 
$\mathrm{Wu}$, Li-chen

$\mathrm{Wu}$, Lili

$\mathrm{Wu}$, Lixin

$\mathrm{Wu}$, Maochun

$\mathrm{Wu}, \mathrm{Min}$

$\mathrm{Wu}$, Ming-Jiuan

Wu, Richard You

$\mathrm{Wu}$, Ruilian

$\mathrm{Wu}$, Shan-Ying

$\mathrm{Wu}$, Shengru

$\mathrm{Wu}$, ShengYun

$\mathrm{Wu}$, Shih-Jeh

$\mathrm{Wu}, \mathrm{Shu}-\mathrm{Ju}$

$\mathrm{Wu}, \mathrm{Xin}$

Wu, Xuedan

Wu, Xuejian

$\mathrm{Wu}$, Yang-Chang

$\mathrm{Wu}$, Yunlong

$\mathrm{Wu}, \mathrm{Yu}$-Tse

Wu, Yu-Wei

$\mathrm{Wu}$, Zucheng

Wulff, Jeremy E.

Wuttke, Stefan

Wybraniec, Sławomir

Wylie, Benjamin J.

Wyman, Ian

Wyszkowski, Mirosław

Xavier, Alencar

Xavier, Ana Maria Rebelo Barreto

Xavier, Cristina Pinto Ribeiro

Xavier, Nuno M.

$\mathrm{Xi}$, Xinping

Xia, Andong

Xia, Chaoming

Xiang, Chunhui

Xiang, Ping

Xiang, Shi-hua

Xiang, Xiaoqiang

Xiao, Kaida

Xiao, Sun

Xiao, Zhengtao

Xiao, Zhousheng

Xie, Bingxian

Xie, Fengwei

Xie, Jing

Xie, Kefeng

Xie, Wankun

Xie, Wei-dong

Xie, Wenyan

Xie, Zhong-Ru

Xin, Dongyue
Xing, Chen

Xing, Fei

Xing, Yalan

Xiong, Jia

Xodo, Luigi

$\mathrm{Xu}$, Airong

$\mathrm{Xu}$, Chungang

$\mathrm{Xu}$, Dong

$\mathrm{Xu}$, Feng

Xu, Fuchao

$\mathrm{Xu}$, Hongtao

$\mathrm{Xu}$, Jialiang

$\mathrm{Xu}$, Jianwei

$\mathrm{Xu}$, Jiaxi

$\mathrm{Xu}$, Jimin

$\mathrm{Xu}$, Jinbin

$\mathrm{Xu}$, Jun

$X u$, Jun-Li

$\mathrm{Xu}$, Kangzhen

$\mathrm{Xu}, \mathrm{Ke}$

Xu, Kui

Xu, Linru

$\mathrm{Xu}, \mathrm{Lu}$

$\mathrm{Xu}, \mathrm{Nan}$

$\mathrm{Xu}$, Peisheng

$\mathrm{Xu}$, Pengtao

$\mathrm{Xu}$, Rong

Xu, Wei

$\mathrm{Xu}$, Wen Tao

$\mathrm{Xu}$, Yan-Ming

$\mathrm{Xu}$, Yi-Jun

$X u$, Yiting

$\mathrm{Xu}$, Zhen-Liang

$\mathrm{Xu}$, Zhimin

Xuan, Tran Dang

Xue, Dongfeng

Xue, Guangai

Yadav, Dhananjay

Yadavalli, Vamsi K

Yaghmur, A.

Yagi, Naoto

Yahagi, Tadahiro

Yajima, Mamiko

Yajima, Shunsuke

Yakhvarov, Dmitry

Yakimova, Luidmila

Yakovlev, Vasily

Yakushev, Vladimir

Yakymovych, Andriy

Yakymovych, Ihor

Yallapu, Murali 
Yamada, Hiroyuki

Yamada, Shigeyuki

Yamagishi, Takehiro

Yamaguchi, Makoto

Yamaguchi, Masahiko

Yamaguchi, Masaki

Yamaguchi, Satoshi

Yamaguchi, Soichiro

Yamaguchi, Yuki

Yamakoshi, Hiroyuki

Yamamoto, Tsuyoshi

Yamamoto, Yasunori

Yamamoto, Yusuke

Yamamura, Soichiro

Yamanaka, Shusuke

Yamaoka, Toshimitsu

Yamaotsu, Noriyuki

Yamato, Takehiko

Yamauchi, Akira

Yamauchi, Satoshi

Yamayoshi, Asako

Yamazaki, Yasuomi

Yamijala, Sharma S. R. K. C.

Yan, Dayun

Yan, Hongbin

Yan, Jing

Yan, Liang

Yan, Lifeng

Yan, Mary

Yan, Sheng

Yan, Yong-Bin

Yan, Yunfeng

Yanase, Emiko

Yanda, Murali

Yáñez, Remedios

Yáñez-Vilar, Susana

Yang, Angela Wei Hong

Yang, Bo

Yang, Chen I.

Yang, Chih-Chung

Yang, Chul-Su

Yang, Chunlong

Yang, Chunzhang

Yang, Ding-Yah

Yang, Feng-Ling

Yang, Fengqing

Yang, Guang

Yang, Guangyu

Yang, Haifeng

Yang, Hoichang

Yang, Hongshun
Yang, Jiale

Yang, Jianxiao

Yang, Jie

Yang, Jing

Yang, Kejia

Yang, Kun

Yang, Lijiang

Yang, Liqun

Yang, Pei-Ming

Yang, Rui

Yang, Seung Hwan

Yang, Shih Chun

Yang, Shun-Chin

Yang, Sung Ho

Yang, Tsung-Shi

Yang, Wei

Yang, Wei-hsiung

Yang, Wen-Chao

Yang, Xin

Yang, Xinghong

Yang, Xinmai

Yang, Xiuwei

Yang, Xuan

Yang, Yang

Yang, Ying

Yang, Ying-Ying

Yang, Yu-Chiao

Yang, Yu-liang

Yang, Zhaogang

Yang, Zhigang

Yannarelli, Gustavo

Yano, Masato

Yanshole, Lyudmila V.

Yao, Guangmin

Yao, Hsien-Tsung

Yao, Jian

Yao, Min

Yao, Zi-Jian

Yaoita, Yasunori

Yaraki, Mohammad Tavakkoli

Yaremenko, Ivan A.

Yaron, Jordan R.

Yashchenok, Alexey

Yasin, Sohail

Yasuda, Kaori

Yasuda, Takako

Yasuhiko, Koga

Yasukawa, Kiyoshi

Yasutake, Yoshiaki

Yates, Matthew

Yatoh, Shigeru 
Yatom, Shurik

Yatsunyk, Liliya

Yavin, Eylon

Yazaki, Ryo

Yazlovitskaya, Eugenia M.

YE, Enyi

Ye, Hailong

Ye, Jian-Hui

Ye, Jianqiao

Ye, Jinxing

Ye, Jun

Ye, Qing

Ye, Shu-feng

Ye, Yunpeng

Yedjou, Clement G.

Yeh, Chen-Yu

Yeh, I-Ju

Yeh, Jwu-Lai

Yeh, Ting-feng

Yeliseev, Alexei

Yelko, Rodríguez-Carrasco

Yen, Chia-Hung

Yen, Feng-Lin

Yen, Frances T.

Yen, Yi-Kuang

Yeo, Syn

Yeoman, Carl

Yepuri, Nageshwar R.

Yi, Myunggi

Yi, Sun-Shin

$\mathrm{Yi}$, Tao

Yi, Yougen

Yi, Young-Su

Yihao, Chen

Yin, Chungen

Yin, Heng

Yin, Hengfu

Yin, Kai

Yin, Kingsley

Yin, Panchao

Yin, Sheng

Yin, Zhouyang

Yo, Tanaka

Yohda, Masafumi

Yokomatsu, Tsutomu

Yokosuka, Akihito

Yokosyo, Kengo

Yokota, Shingo

Yokoyama, Chikako

Yong, Kar Wey

Yong, Yang-chun
Yoo, Dong Jin

Yoo, Dongwon

Yoo, Hah Young

Yoo, Jung Sun

Yoo, So Young

Yoon, Byung-Il

Yoon, Il

Yoon, In Soo

Yoon, Kee-Dong

Yoon, Moon-Young

Yoon, Seong-Jun

Yoon, Tae Jun

Yoon, Yeojoon

Yoshida, Eri

Yoshida, Kentaro

Yoshida, Nidia Cristiane

Yoshida, Takashi

Yoshihara, Eiji

Yoshimatsu, Mitsuhiro

Yoshimi, Yasuharu

Yoshimitsu, Takehiko

Yoshimoto, Francis K.

Yoshimune, Miki

Yoshimura, Tomokazu

Yoshinaga, Jun

Yoshinari, Nobuto

Yoshino, Hironori

Yoshioka, Ken-ichi

Yoshioka, Taiyo

You, Hye Jin

You, Jungmok

You, Seungkwon

You, Zongbing

Young, Michael

Yousefi, Behrooz H.

Youssef, Hibaoui

Yperman, Jan

Yu, Fabiao

$\mathrm{Yu}$, Hua

$\mathrm{Yu}$, Jaecheul

Yu, Jae-sik

$\mathrm{Yu}$, Jingang

$\mathrm{Yu}$, Jingxian

$\mathrm{Yu}$, Kang

$\mathrm{Yu}$, Leixiao

$\mathrm{Yu}$, Liang

Yu, Ling

$\mathrm{Yu}, \mathrm{Lu}$

$\mathrm{Yu}$, Meihua

$\mathrm{Yu}$, Rongmin

$\mathrm{Yu}$, Sheng-Sheng 


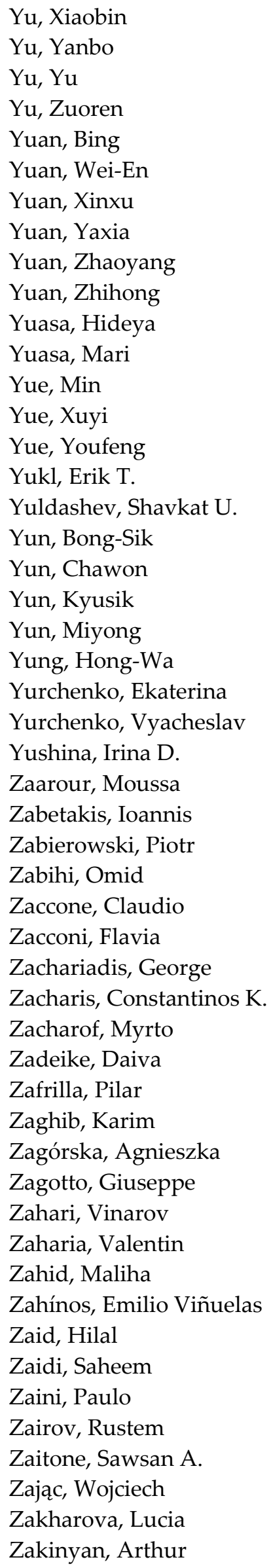

Zakłos-Szyda, Malgorzata

Zakoldaev, Roman A.

Zakrevsky, Paul

Zakrzewska, Malgorzata

Zakrzewski, Jerzy

Zalibera, Michal

Zaltariov, Mirela-Fernanda

Załuski, Daniel

Zamaraewa, Maria

Zamaratskaia, Galia

Zamay, Tatiana

Zambito, Ylenia

Zamborine-Nemeth, Eva

Zambrano-Zaragoza, Maria L.

Zamfir, Carmen Lăcrămioara

Zammit-Mangion, Marion

Żamojć, Krzysztof

Zamora-Ros, Raul

Zamyatnin, Andrey

Zanardi, Emanuela

Zandvliet, Harold

Zanetti, Stefania

Zangrando, Ennio

Zani, Lorenzo

Zannetti, Antonella

Zanolla, Marianela

Zanotti, Giuseppe

Zapata, Paula

Zapico, Sara C.

Zappia, Giovanni

Zaprutko, Lucjusz

Zaptata-Torres, Molecular Modeling Gerald

Zarabadi-Poor, Pezhman

Zaragoza Contreras, Erasto Armando

Zareba, Jan K.

Zarejousheghani, Mashaalah

Zaremba-Czogalla, Magdalena

Zarkovic, Neven

Żarowska, Barbara

Zarrelli, Armando

Zarzycki, Pawel K.

Zarzyka, Iwona

Zastrow, Melissa L.

Zatterale, Federica

Zavala-Rivera, Paul

Zawada, Katarzyna

Zawierucha, Iwona

Zbancioc, Gheorghiță

Zdanowicz, Magdalena

Zdarta, Jakub

Zdenek, Christina 
Zechel, Stefan

Zedler, Łukasz

Zeeshan, Muhammad

Zehl, Martin

Zeković, Zoran

Żelaszczyk, Dorota

Zeleny, Reinhard

Zelkó, Romána

Zeng, Bijun

Zeng, Huiping

Zeng, Jia

Zeng, Tao

Zeng, Weiguang

Zeng, Xiangkang

Zengin, Gokhan

Zeni, Olga

Zepeda, Rossana C.

Zeremski, Tijana

Żesławska, Ewa

Zestos, Alexander G.

Zettsu, Nobuyuki

Zha, Gaofeng

Zhai, Kui

Zhan, Peng

Zhan, Wenbo

Zhan, Xuanzhi

Zhang, Bo

Zhang, Cankui

Zhang, Chao

Zhang, Chengbin

Zhang, Chunhui

Zhang, Dongming

Zhang, Dongzhou

Zhang, En

Zhang, Fan

Zhang, Fuming

Zhang, Guannan

Zhang, Guoqi

Zhang, Hongbo

Zhang, Hongyi

Zhang, $\mathrm{Hu}$

Zhang, Huawei

Zhang, Huiying

Zhang, Jianying

Zhang, Jianyong

Zhang, Jie

Zhang, Jin

Zhang, JingJing

Zhang, Jinwei

Zhang, Junbo

Zhang, Junliang
Zhang, Junxian

Zhang, Junzeng

Zhang, Kai

Zhang, Kaihuan

Zhang, Lei

Zhang, Li

Zhang, Liangren

Zhang, Liqiang

Zhang, Peng

Zhang, Qian

Zhang, Qiangzhe

Zhang, Qichun

Zhang, Qingrui

Zhang, Qing-Wen

Zhang, Qiong

Zhang, Run

Zhang, Shanju

Zhang, Shuang-Qing

Zhang, Tao

Zhang, Tong

Zhang, Wei

Zhang, Wen

Zhang, Wencai

Zhang, Wenjun

Zhang, Wenxuan

Zhang, Wenzhong

Zhang, Xiang

Zhang, Xiaoning

Zhang, Xiaoyu

Zhang, Xinxing

Zhang, Xinyu

Zhang, Xu

Zhang, Xuexin

Zhang, Yancheng

Zhang, Ye-Wang

Zhang, Yinfeng

Zhang, Yixuan

Zhang, Yonghong

Zhang, Youjun

Zhang, Youming

Zhang, Yu

Zhang, Yue

Zhang, Yumiao

Zhang, Yuning

Zhang, Yuyu

Zhang, Zhanhui

Zhang, Zhenbin

Zhang, Zhenhua

Zhang, Zhidong

Zhang, Zhiguo

Zhang, Zhong-Xing 
Zhang, Zongtao

Zhao, Boxin

Zhao, Fengyu

Zhao, Fuli

Zhao, Jinling

Zhao, Linbo

Zhao, Liyan

Zhao, Ningning

Zhao, Peishen

Zhao, Qihua

Zhao, Qingnan

Zhao, Wanxiang

Zhao, Wei

Zhao, Weijie

Zhao, Xi

Zhao, Xianhui

Zhao, Xiuhua

Zhao, Xuebing

Zhao, Xuhui

Zhao, Yanchuan

Zhao, Yong-Biao

Zhao, Yuguang

Zhao, Yunfeng

Zhao, Yuxia

Zhao, Zengfeng

Zhao, Zhenjiang

Zhao, Zhenwen

Zhdanov, Andrey P.

Zheleva-Dimitrova, Dimitrina

Zheng, Bo

Zheng, Liqiu

Zheng, Shilong

Zheng, Shuanghao

Zheng, Xing

Zhiponova, Miroslava

Zhitomirsky, Igor

Zhivkova, Zvetanka

Zhong, Haizhen Andrew

Zhongfeng, Zhang

Zhou, Beiyan

Zhou, Chi

Zhou, Dongfang

Zhou, Guangfeng

Zhou, Hai-bing

Zhou, Hua

Zhou, Jiajing

Zhou, Jian

Zhou, Li

Zhou, Linli

Zhou, Mei

Zhou, Phoebe
Zhou, Qingyu

Zhou, Qun

Zhou, Shengbin

Zhou, Tao

Zhou, Teng

Zhou, Xiaochun

Zhou, Xiaohong

Zhou, Xinglin

Zhou, Yan

Zhou, Zhe

Zhou, Zhidong

Zhu, Caihong

Zhu, Chenglin

Zhu, Dandan

Zhu, Feng

Zhu, Hui

Zhu, Jie

Zhu, Junjie

Zhu, Lan

Zhu, Lin

Zhu, Qingzeng

Zhu, Wei

Zhu, Weigang

Zhu, Wufu

Zhu, Xuejun

Zhu, Yuyang

Zhu, Zhenbao

Zhuang, Shu-lin

Zhuang, Yongliang

Zhukov, Igor

Zhukova, Natalia

Zhuo, Guan-Yu

Ziaee, Ahmad

Ziarno, Małgorzata

Ziats, Nicholas P.

Zidek, Jan

Žídek, Lukáš

Zidorn, Christian

Zięba, Andrzej

Ziegenbalg, Dirk

Zielinska, Aleksandra

Zielinska, Danuta

Zielińska, Ewelina

Zielińska, Sylwia

Zielińska-Błajet, Mariola

Zielińska-Pisklak, Monika

Ziembińska-Buczyńska, Aleksandra

Ziemkowska, Wanda

Zienkiewicz-Strzałka, Małgorzata

Zierkiewicz, Wiktor

Zilberg, Shmuel 
Zille, Andrea

Zimmerman, William

Zimmermann, Benno

Zimnyakov, Dmitry

Zinchenko, Anatoly

Zingg, Jean-Marc

Zinicovscaia, Inga

Zitko, Jan

Zivanovic, Jasmina

Ziyatdinova, Guzel

Zizza, Pasquale

Zlatopolskiy, Boris

Zloh, Mire

Złotek, Urszula

Zlotin, Sergey G.

Zmora, Pawel

Zmudzki, Pawel

Znamirowska, Agata

Znosko, Brent M.

Zobel, Patrick

Zobi, Fabio

Zoccali, Mariosimone

Zoidis, Grigoris

Żołek, Teresa

Żołnowska, Beata

Zoltan-Istvan, Szabo

Zonta, Cristiano

Zoran, Stević
Zoratti, Mario

Zorc, Branka

Zordoky, Beshay

Zorov, Dmitry B.

Zou, Gang

Zoumpoulakis, Panagiotis

Zoumpourlis, Vassilis

Zovko Končić, Marijana

$\mathrm{Zu}$, Youli

Zubarev, Roman

Zubitur, Manoli M.

Zubko, Maciej

Zubkova, Olga

Zubrienè, Asta

Zubrik, Anton

Zucca, Antonio

Žugić, Ana

Zuk, Magdalena

Zuluaga, Paola

Zuniga, Cristal

Zuorro, Antonio

Zupko, Istvan

Zuza, Ester

Zwacka, Ralf

Zwergel, Clemens

Zygiridis, Theodoros

Żywicka, Anna

Żyżelewicz, Dorota 